Portland State University

PDXScholar

6-2-1997

\title{
The Column Multiplicity Problem in Decomposition of Functions and Relations
}

Rahul Malvi

Portland State University

Follow this and additional works at: https://pdxscholar.library.pdx.edu/open_access_etds

Part of the Electrical and Computer Engineering Commons Let us know how access to this document benefits you.

Recommended Citation

Malvi, Rahul, "The Column Multiplicity Problem in Decomposition of Functions and Relations" (1997). Dissertations and Theses. Paper 5830.

https://doi.org/10.15760/etd.7701

This Thesis is brought to you for free and open access. It has been accepted for inclusion in Dissertations and Theses by an authorized administrator of PDXScholar. Please contact us if we can make this document more accessible: pdxscholar@pdx.edu. 


\section{THESIS APPROVAL}

The abstract and thesis of Rahul Malvi for the Master of Science in Electrical and Computer Engineering were presented June 2nd 1997, and accepted by the thesis committee and the department.

COMMITTEE APPROVALS:

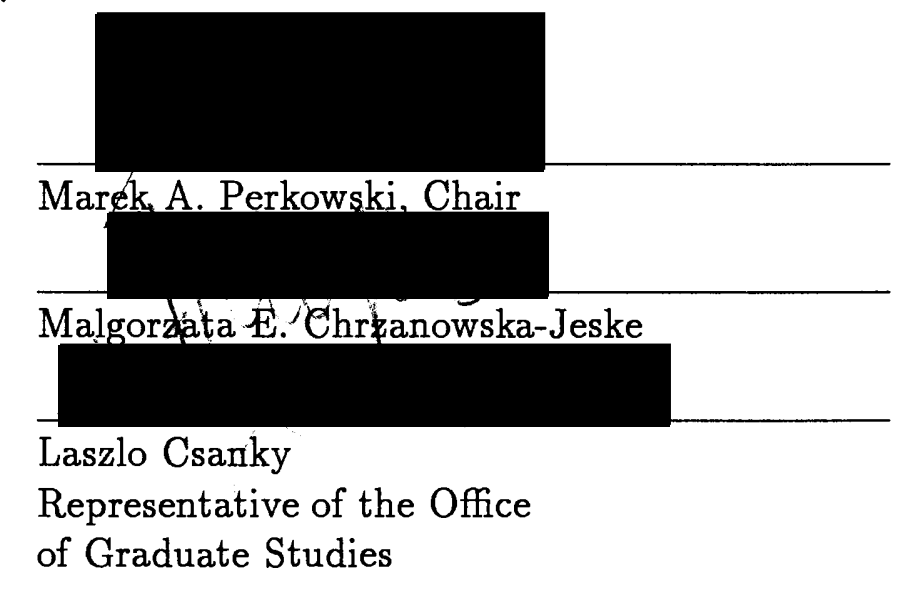

DEPARTMENT APPROVAL:

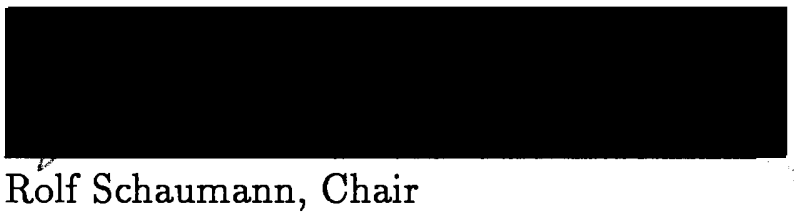

Department of Electrical Engineering

ACCEPTED FOR PORTLAND STATE UNIVERSITY BY THE LIBRARY

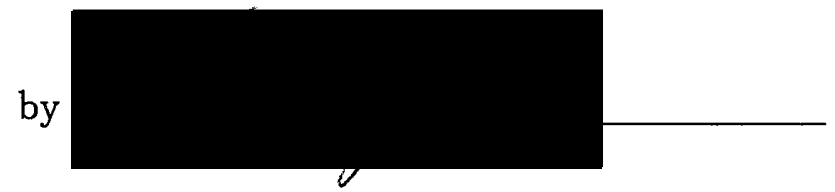

on

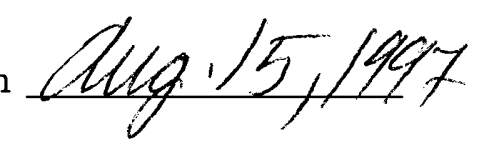


An abstract of the thesis of Rahul Malvi for the Master of Science in Electrical and Computer Engineering presented June 2nd 1997.

Title: The Column Multiplicity Problem in Decomposition of Functions and Relations

Finding the column multiplicity in Functional Decomposition has been known to be one of the most important problems to be solved in the process of functional decomposition of discrete functions. A lot of research has been done in this field with many new heuristics generated to find the column multiplicity, but there has not been an evaluation of the algorithms on the kinds of graphs that occur in decomposition and whether having an exact method to calculate the column multiplicity is useful from the overall design goals. The intent of this thesis was to investigate the column multiplicity problem, in order to find out how different heuristic algorithms compare with an exact algorithm for finding the column multiplicity, and to find out if an exact graph coloring is actually required or not.

In order to investigate this problem of column multiplicity in functional decomposition, two graph coloring programs, and a multi-coloring program were written: one of the graph coloring programs is an exact graph coloring, the other graph coloring program and the multi-coloring program are both based on heuristic algorithms. The two graph coloring programs have been compared in this thesis on randomly generated graphs. These programs were incorporated into the multi-valued decomposer of functions and relations $M V G U D$ which was developed at Portland State University. Extensive testing of MVGUD with these graph coloring and multi-coloring programs has been done in this thesis. MVGUD was tested on both circuit benchmarks and on machine learning benchmarks. 
THE COLUMN MULTIPLICITY PROBLEM IN DECOMPOSITION OF FUNCTIONS AND RELATIONS

\author{
by \\ RAHUL MALVI
}

A thesis submitted for the partial fulfillment of the requirements for the degree of

\author{
MASTER OF SCIENCE \\ in \\ ELECTRICAL AND COMPUTER ENGINEERING
}

Portland State University

1997 


\section{ACKNOWLEDGEMENTS}

I would like to thank Dr. Marek A. Perkowski, my advisor, who introduced me to the areas of Logic Synthesis, and Decomposition of Functions and Relations. This thesis would not have been possible without the guidance and encouragement I received from Dr. Perkowski. I would also like to thank Dr. Malgorzata E. Chrzanowska-Jeske and Dr Laszlo Csanky for their valuable comments which helped to improve this thesis.

I would also like to thank my parents for their support and encouragement, and I would like to thank Uju for her constant support and encouragement. Also, I would like to thank Shirley, Laura and Ellen for all their assistance and support. 
CONTENTS

LIST OF TABLES V v

LIST OF FIGURES vii

1 INTRODUCTION 1

2 GENERAL DECOMPOSITION OF BOOLEAN FUNCTIONS 5

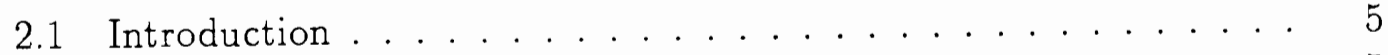

2.2 Generalized Functional Decomposition . . . . . . . . . . 5

2.3 Basic Notions and Definitions . . . . . . . . . . . . . . 6

2.3.1 A Karnaugh Map vs a Decomposition Chart . . . . . . . . 6

2.3.2 A Minterm ................. 6

2.3 .3 A Cube ..................... 7

2.3.4 Hamming Distance . . . . . . . . . . . . . . 7

2.3.5 The Cofactor of a Function ............. . . . 8

2.3.6 The Free Set and the Bound Set............. 8

2.3.7 Compatible and Incompatible Columns of a Decomposition Chart .................. 8

2.3.8 Column Multiplicity . . . . . . . . . . . . . . 9

2.3.9 A Compatibility graph vs an Incompatibility graph . . . . 9

2.3.10 Ashenhurst and Curtis Decompositions . . . . . . . . . . 9

2.4 The Steps involved in performing a Curtis Decomposition . . . . . 11

2.5 Applications of Functional Decomposition . . . . . . . . . . 14

3 SOME NEW APPROACHES TO GRAPH COLORING: TWO PROGRAMS AND TWO IDEAS 15

3.1 Basic Notions and Definitions . . . . . . . . . . . . . 16

3.1.1 Dominations in an Incompatibility Graph . . . . . . . . 16

3.1.2 A Cyclic Graph and Cycles in an Incompatibility Graph . 18

3.1.3 A Complete Graph . . . . . . . . . . . . . . . . . . . . . 18

3.2 A New Approach to a Heuristic Graph Coloring Using Dominations 19

3.2.1 An Example showing how DOM colors a non cyclic graph . 19

3.2.2 An Example showing how DOM colors a cyclic Graph . . . 21

3.2.3 Different Graphs Possible in Decomposition, and how they are handled by DOM .............. . . 23 
3.2.4 Implementation Details of DOM . . . . . . . . . 25

3.2.5 The strong and weak points of DOM . . . . . . 27

3.3 An idea which extends DOM to an Exact Graph Coloring . . . . . 29

3.3.1 Algorithm for EXDOM . . . . . . . . . . . 31

3.4 A New approach to an Exact Graph Coloring . . . . . . . . 35

3.4.1 The Exact Graph Coloring Program Implementation Details 36

3.4.2 An Example showing how EXOC colors a graph . . . . . 38

3.4 .3 Algorithm for EXOC . . . . . . . . . . . . . 41

3.4.4 Future Possible Improvements in EXOC . . . . . . . . 47

3.5 A New idea for an Exact Graph Coloring Program combining both $D O M$ and $E X O C \ldots \ldots \ldots \ldots \ldots . \ldots . \ldots . \ldots 49$

3.5.1 Example showing how DOMEXOC colors a graph with cycles 49

3.5 .2 Algorithm for DOMEXOC . . . . . . . . . . . 51

3.6 Summary and Conclusions of Chapter $3 \ldots \ldots \ldots \ldots$

4 A COMPARISON OF THE DOM AND EXOC GRAPH COLORING PROGRAMS ON RANDOM GRAPHS $\mathbf{5 4}$

4.1 Results of running DOM on Randomly generated Graphs . . . . 54

4.2 Comparison of DOM and EXOC on Randomly generated Graphs 56

4.2 .1 Notations used in the Tables . . . . . . . . . . . . 56

4.3 Summary and Conclusions of Chapter $4 \ldots \ldots \ldots$

5 AN EVALUATION OF THE PROBLEM OF COLUMN MULTIPLICITY IN DECOMPOSITION OF FUNCTIONS 62

5.1 Some Notions and Definitions . . . . . . . . . . . . 63

5.1 .1 A Vacuous Variable . . . . . . . . . . . . . . . 63

5.1 .2 Clique Partitioning and Clique Covering . . . . . . . 63

5.1.3 Clique Covering of a Compatibility Graph . . . . . . . . 64

5.1 .4 Decomposed Function Cardinality . . . . . . . . . . 65

5.2 An Introduction to MVGUD . . . . . . . . . . . . . . . 66

5.2.1 Strategy used by $M V G U D$ to perform Decompositions . . 66

5.3 A Comparison of the different Strategies of finding the Column

Multiplicity in Functional Decomposition . . . . . . . . . . . 68

5.3 .1 Notations Used in the Tables . . . . . . . . . . . 68

5.3.2 A Comparison of the different Strategies of finding the Column Multiplicity on MCNC Benchmarks . . . . . . . . 69

5.4 A Comparison of the different Strategies of finding the Column Multiplicity on Machine Learning Benchmarks . . . . . . . . . 75

5.4.1 A Summary of the Results Obtained from Testing on Machine Learning Benchmarks . . . . . . . . . . . . 80 
5.5 Some Questions and Conclusions Reached from the Results of the Testing . . . . . . . . . . . . . . . . 84

5.6 What is the next step to solve the Column Multiplicity Problem . 85

6 A NEW APPROACH TO MULTI-COLORING USING DOMINATIONS: COLUMN COMPATIBILITY FOR FUNCTIONS AND RELATIONS

6.1 Basic Notions and Definitions . . . . . . . . . . . 86

6.1.1 Multi-Coloring ................ 86

6.1.2 Maximum Independent Sets in an Incompatibility Graph . 86

6.2 A Comparison showing the Equivalence of a Multi-Coloring and a Maximum Clique Covering . . . . . . . . . . . . . . . 86

6.3 Why it was decided to use a Multi-Coloring in Decomposition . 87

6.3.1 An Introduction to Multi-Valued Relations . . . . . . . . 88

6.3.2 An Example showing Decomposition of Multiple-Valued Relations .................... . 91

6.3.3 An Example showing Decomposition of a Binary Function and the creation of Relations ............ . . 93

6.4 A New Approach to Multi-Coloring using Dominations . . . . . 97

6.4.1 Example showing how MISDOM colors a non cyclic Graph 99

6.4.2 Implementation Details for MISDOM . . . . . . . . . . 100

6.4.3 Details of the Multi-Coloring Program, MISDOM . . . . 101

6.4.4 Some Features of MISDOM . . . . . . . . . . . . . 104

6.5 Summary and Conclusions of Chapter $6 \ldots \ldots \ldots$

7 AN EVALUATION OF THE MULTI-COLORING PROGRAM107

7.1 An Evaluation of Running MISDOM on randomly generated graphs 107

7.2 A New Approach to calculate the cost function of a Decomposed

Function or Relation . . . . . . . . . . . . . . . . . 109

7.2.1 Calculating NOFP for a Relation and a Function . . . . 110

7.2.2 Calculating COSC for a Relation and a Function . . . . . 112

7.3 An Evaluation of Running MISDOM on Graphs generated during Decomposition of Functions and Relations . . . . . . . . . 115

7.3.1 A Comparison of MISDOM and DOM on MCNC Benchmarks 116

7.3.2 A Comparison of MISDOM and DOM on Machine Learning Benchmarks . . . . . . . . . . . . . . 119

7.3.3 A Summary of the results of testing MISDOM on Machine Learning Benchmarks. . . . . . . . . . . . . . 129

7.4 Summary and some Conclusions based on the results obtained in Chapter $7 \ldots \ldots \ldots \ldots$. . . . . . . . . . . 134 
8 MY CONTRIBUTIONS TO THE FUNCTIONAL DECOMPOSITION GROUP, CONCLUSIONS AND FUTURE WORK 138 BIBLIOGRAPHY 


\section{LIST OF TABLES}

2.1 Table showing Minterms and Cubes . . . . . . . . . . 7

4.1 Nodes vs Edge Percent for DOM on randomly generated graphs . 55

4.2 Comparison of EXOC and DOM on randomly generated graphs with edge percent from 10 to $35 \ldots \ldots \ldots \ldots 7$

4.3 Comparison of EXOC and DOM on randomly generated graphs with edge percent from 40 to $90 \ldots \ldots \ldots \ldots$

5.1 A Comparison of the results obtained by running $M V G U D$ with 2 variables in the Bound Set on MCNC Benchmarks . . . . . . 70

5.2 A Comparison of results obtained by running $M V G U D$ with 4 variables in the Bound Set on MCNC Benchmarks . . . . . . . .

5.3 A Comparison of results obtained by running $M V G U D$ with 5 variables in the Bound Set on MCNC Benchmarks . . . . . . . . 72

5.4 A Comparison of results obtained by running $M V G U D$ with 2 variables in the Bound Set on 8 variable MLB . . . . . . . 76

5.5 A Comparison of results obtained by running $M V G U D$ with 4 variables in the Bound Set on 8 variable MLB . . . . . . . . . 77

5.6 A Comparison of results obtained by running $M V G U D$ with 5 variables in the Bound Set on 8 variable MLB . . . . . . 78

5.7 A Comparison of results obtained by running $M V G U D$ with 2 variables in the Bound Set on 12 variable MLB . . . . . . . . 79

5.8 A Comparison of the Total Colors obtained by running $M V G U D$ on Machine Learning Benchmarks . . . . . . . . . . . . . . . 82

5.9 A Comparison of Total Colors generated by DOM, and CLIP compared with total colors generated by $E X O C$ on the same graphs for two, four and five variables in the Bound Set for Machine Learning Benchmarks . . . . . . . . . . . . . . . . 83

5.10 A Summary showing the Addition of the Total Colors obtained in Table 5.9

6.1 Table showing Relations in a four binary input and one multioutput Function . . . . . . . . . . . . . 9 90

7.1 An Evaluation of running MISDOM on Randomly generated graphs 108

7.2 MVGUD run with 2 variables in the bound set on MCNC Benchmarks 
7.3 MVGUD run with 4 variables in the bound set on MCNC Benchmarks ...................... . . 117

7.4 MVGUD run with 5 variables in the bound set on MCNC Benchmarks . . . . . . . . . . . . . . . . . . . . 117

7.5 MVGUD run with 2 variables in the bound set on 8 variable Machine Learning Benchmarks with $70 \%$ don't cares . . . . . . 120

7.6 MVGUD run with 4 variables in the bound set on 8 variable Machine Learning Benchmarks with $70 \%$ don't cares . . . . . . . . 121

7.7 MVGUD run with 5 variables in the bound set on 8 variable Machine Learning Benchmarks with $70 \%$ don't cares . . . . . . . 122

7.8 MVGUD run with 6 variables in the bound set on 8 variable Machine Learning Benchmarks with $70 \%$ don't cares . . . . . . . 123

$7.9 M V G U D$ run with 2 variables in the bound set on 8 variable Machine Learning Benchmarks with $90 \%$ don't cares . . . . . . . 125

7.10 MVGUD run with 4 variables in the bound set on 8 variable Machine Learning Benchmarks with $90 \%$ don't cares . . . . . . . 126

7.11 MVGUD run with 5 variables in the bound set on 8 variable Machine Learning Benchmarks with $90 \%$ don't cares . . . . . . 127

7.12 MVGUD run with 6 variables in the bound set on 8 variable Machine Learning Benchmarks with $90 \%$ don't cares . . . . . . . 128

7.13 Resulting G Function for MVGUD run on Benchmark psu_ch $47 f 0 \quad 130$

7.14 Resulting H Function for MVGUD run on Benchmark psu_ch47fo 131

7.15 Resulting G Function for MVGUD run on Benchmark psu_rnd3 . 132

7.16 Resulting H Function for MVGUD run on Benchmark psu_rnd3 . 133 


\section{LIST OF FIGURES}

2.1 Difference between Disjoint and Non Disjoint Decompositions . . 5

2.2 A Kmap vs a Decomposition Chart . . . . . . . . . . . . . . 6

2.3 A Compatibility graph and an Incompatibility graph of a Function 10

2.4 An Ashenhurst Decomposition . . . . . . . . . . . . . . . 10

2.5 A Curtis Decomposition ... . . . . . . . . . . . . 11

2.6 The Steps involved in Performing a Curtis Decomposition. . . . . 12

3.1 Dominations and Pseudo Dominations in an Incompatibility Graph 17

3.2 A Cyclic Graph . . . . . . . . . . . . . . . . . . . 18

3.3 A Complete Graph . . . . . . . . . . . . . . . . . 18

3.4 Example showing how $D O M$ colors a non cyclic graph . . . . . 20

3.5 Example showing how DOM colors a cyclic graph . . . . . . . 22

3.6 The Different Classes of Incompatibility Graphs that may be generated in the steps of Functional Decomposition . . . . . . . . 24

3.7 The tree Search showing how DOM can be made exact when it encounters a cyclic graph . . . . . . . . . . . . . 30

3.8 Data Structure for a Stack Node used in the Exact Graph Coloring Program ......................... 36

3.9 Tree Search for the Exact Graph Coloring Algorithm . . . . . . 39

3.10 An Example showing how DOMEXOC colors a cyclic graph . . . 50

4.1 Plot of Nodes vs Time for DOM on graphs with increasing number of nodes with different percent of Edges . . . . . . . . 56

4.2 Graph of Nodes vs Time showing how EXOC performs on randomly generated graphs with edge percent from $30 \%$ to $70 \%$. . . 59

4.3 Summary of Results showing how close to exact are the solutions generated by DOM on randomly generated graphs . . . . . 60

5.1 The Karnaugh map of a function showing a Vacuous Variable . . 63

5.2 The Difference between Clique Covering and Clique Partitioning . 64

5.3 Calculation of DFC in Binary and Multi-Valued Functions . . . 65

6.1 Difference between Clique Covering and Multi-Coloring . . . . . 87

6.2 An Example showing Decomposition of Multiple-Valued Relations 92

6.3 An Example showing Decomposition of a Binary Function and the Creation of Relations . . . . . . . . . . . . . . . 93 
6.4 Decomposing a Function and using a Multi-Coloring to produce $\mathrm{G}$ Relations and $\mathrm{H}$ functions . . . . . . . . . . . . . . 94

6.5 The Difference Decompositions obtained by using a Graph Coloring or a Multi-Coloring while Decomposing a Relation . . . . . . 96

6.6 An Example showing how MISDOM colors a non cyclic Graph . . 98

6.7 Data Structure used for a Node of the Incompatibility Graph . . . 100

6.8 Reason why a dominated node can be colored all the colors of the nodes which dominate it . . . . . . . . . . . 105

7.1 Different classes of Functions possible from a Relation . . . . . . . 110

7.2 A Comparison of the calculation of NOFP for a Relation and for a Function . . . . . . . . . . . . . . . . 111

7.3 A Comparison showing that more repetions of common variables in not necessarily better . . . . . . . . . . . . 113

7.4 Example showing how $C O S C$ is calculated . . . . . . . . 114

7.5 A Graph for 8 variable Machine Learning Benchmarks with $70 \%$ don't cares showing how NOFP and COSC vary with increasing number of variables in the Bound set . . . . . . . . . . 135

7.6 A Graph for 8 variable Machine Learning Benchmarks with $90 \%$ don't cares showing how $N O F P$ and $C O S C$ vary with increasing number of variables in the Bound set . . . . . . . . . 136 


\section{CHAPTER 1}

\section{INTRODUCTION}

Functional Decomposition is one of the most general methods to solve engineering and science optimization problems. Functional Decomposition is an NP-complete problem. Because of its low time efficiency it is rarely used in industry, while Algebraic Factorization and Binary Decision Diagrams are used instead. Recently though, there has been some strong renewed interest in using Functional Decomposition for applications like FPGA mapping and PLA Decomposition. Functional Decomposition involves breaking down a function of larger complexity into smaller blocks of less complexity and then these smaller blocks can be broken down themselves. The Functional Decomposition group at Portland State University was doing this research for Wright Patterson Air Force base and for Abtech Corporation. The Pattern Theory group (PTG) at the Avionics Laboratory of Wright Laboratories, at Wright Patterson Air Force Base develops new system-level concepts for military applications, mostly based on Machine Learning and Image Processing Technologies. Machine Learning is making the machine learn(induce concept descriptions) as much as possible with as little data as possible. Hence the idea is that the machine must train on the set of samples and find the pattern in the set of samples. Then the machine can apply the pattern to all the data and thus recreate the values of the original samples not given to it. Most, if not all, functions taken from real-life applications have been found to have some pattern. There are many methods which can be used in Machine Learning, like, Neural Nets, Fuzzy Logic, Decision Trees, Binary Decision Diagrams(BDDs), Functional Decision Diagrams(FDDs), Kronecker Decision Diagrams(KDDs), Sum of Products(SOP), e.t.c. All of these methods are 
computationally expensive, but usually less than the Curtis Decomposition. The approach of the PTG is based on Logic Synthesis methods: predominantly the Curtis Decomposition of Boolean functions [16]. The new approach of the PTG will allow both automatic learning of any kind of images, and automatic creation of algorithms, from examples of their behavior. The PTG has developed the programming system FLASH [7], which uses many Decomposition ideas, and is a Testbed for machine learning based on the logic synthesis approach. The applications considered by Wright Laboratories include Pattern Recognition, Algorithm Creation, Data Compression, Machine Learning and Logic Minimization. Functional Decomposition is the approach used by the group at Portland State University. Functional Decomposition can be applied, to minimize a function by finding a pattern in it, and thus breaking the original function into smaller functions. The structure of the decomposed blocks constitutes the algorithm of the function and thus the decomposition finds the pattern in the function. These smaller functions or sub-functions are the "features" or "concepts" found in the original function

Over the last twenty years a lot of effort has gone into the many synthesis methods like BDDs, SOP e.t.c. and it has been found that these methods achieve good results $80 \%$ of the time and are fast, while the Ashenhurst [1] or the Curtis [2] Decompositions will achieve good results nearly $100 \%$ of the time but are very slow. The difficulty of applying the Ashenhurst/Curtis Decomposition is that, as the number of input variables and terms increases, the Decomposition time increases exponentially. Hence we must be able to reduce the computational time to an acceptable level for a given number of variables(10 to 30 variables), while sacrificing a very small amount of solution complexity $(2 \%$ to $3 \%)$. In many industrial and military applications it has been found that the data typically consist of a very large number of don't cares, typically more than $99 \%$. Hence the goal of this research was to develop a Decomposer capable of decomposing functions with many input variables, but with a very large percentage of don't 
cares. Also, in this research we wanted to use different algorithms or techniques such as Graph Coloring, Maximum Cliques, Compatibility and Incompatibility Graphs, both exact and approximate, and test the various heuristics and exact methods in conjunction with the Ashenhurst/Curtis Decomposition method in order to decompose problems much more efficiently and without losing much in accuracy.

Functional Decomposition involves three basic steps namely Variable Partitioning, calculating Column Multiplicity and Encoding. A lot of research has been done in Functional Decomposition, but it is still not known how important each step is and whether one step is more important than the other. Two Functional Decomposers were developed at Portland State University: one a multjvalued Decomposer MVGUD [26] and the other a binary Decomposer GUD [26]. $G U D$ stands for General Universal Decomposer. GUD was in turn put into a larger program called Multis which also had two other Functional Decomposers: TRADE [5,6] and DEMAIN [4] incorporated in it. Multis is capable of using different Decomposers at different steps of the Decomposition thus making it possible to combine different strategies, while decomposing a function.

This thesis deals with the Column Multiplicity problem in Functional Decomposition. There are basically four methods to solve the Column Multiplicity Problem in Functional Decomposition: Set Covering, Graph Coloring, Clique Partitioning and Clique Covering. All these are NP-complete problems. Chapter 2 of this thesis gives an introduction to Decomposition of Boolean Functions. Chapter 3 presents two new Graph Coloring programs: one a Heuristic method, and the other an Exact method. Also in Chapter 3 ideas for two new exact Graph Coloring programs are presented. In Chapter 4 the heuristic Graph Coloring method is compared with the exact Graph Coloring method on graphs generated randomly, in order to see how these two programs compare in terms of their computational time, and how close to exact are the solutions generated by the heuristic Graph Coloring program. The two programs have been incorporated into the Functional 
Decomposer MVGUD, and this decomposer already has a Greedy Clique Partitioning incorporated into it. So in Chapter 5 all three methods are compared, by testing them on functions generated from real life problems, to see how well these programs solve the Column Multiplicity problem. The testing was done on two types of Benchmarks: $M C N C$ benchmarks, generated from Circuit minimization, and Machine Learning Benchmarks from areas of Pattern Recognition, Machine Learning and Knowledge Discovery in Databases. In Chapter 6 the concept of decomposition of relations is introduced, and in this Chapter a new heuristic Multi-Coloring program is presented. In Chapter 7 this Multi-Coloring program is tested in Decomposition of Functions and Relations in order to see if the Multi-Coloring can solve the Column Multiplicity problem better than a Graph Coloring or a Clique Partitioning. Finally, Chapter 8 summarizes the work and addresses my point of view of what the future tasks of the Functional Decomposition group at Portland State University should be. 


\section{CHAPTER 2}

\section{GENERAL DECOMPOSITION OF BOOLEAN FUNCTIONS}

\subsection{Introduction}

Decomposition is a method to break down a large function into a number of smaller functions, which are easier to analyze and realize than the large input function. Boolean Decomposition uses a Boolean representation. Many different kinds of Decompositions have been developed. The first was the Ashenhurst Decomposition defined in [1], then Curtis [2] extended the Ashenhurst Decomposition, making it more general. This thesis deals with the Curtis decomposition, and in this Chapter, the Curtis Decomposition will be presented.

\subsection{Generalized Functional Decomposition}

The Boolean Decomposition involves breaking a function $F(A, B)$ into $H(g(B)$ , $A$ ) where the number of inputs of $H$ is smaller than that of $F$. If $A \cap B=\emptyset$ then it is called a Disjoint Decomposition, as shown in Figure 2.1(a), else it is

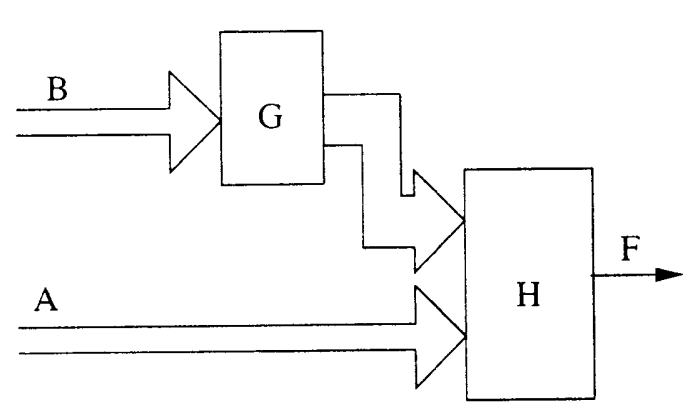

(a) A Disjoint Decomposition

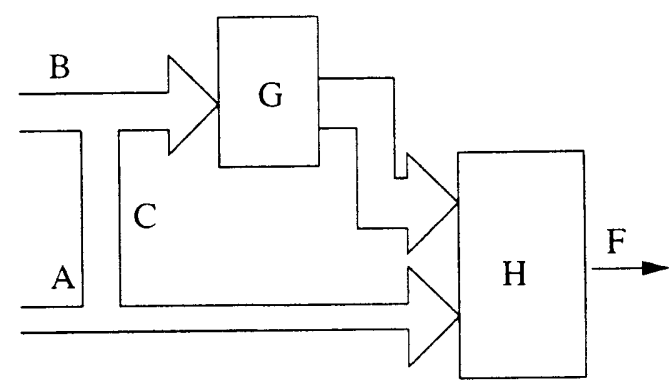

(b) A Non Disjoint Decomposition

Figure 2.1: Difference between Disjoint and Non Disjoint Decompositions 


\begin{tabular}{|c|c|c|c|c|}
\hline $\mathrm{ab}$ & 00 & 01 & 11 & 10 \\
\hline 00 & $X$ & 0 & 0 & 1 \\
\hline 01 & $X$ & $X$ & 0 & 1 \\
\hline 11 & 0 & 0 & $X$ & 0 \\
\hline 10 & 0 & 1 & 1 & 0 \\
\hline
\end{tabular}

(a) A Karnaugh Map

\begin{tabular}{|c|c|c|c|c|}
\hline b & 00 & 01 & 10 & 11 \\
\hline 00 & $\mathrm{X}$ & 0 & 1 & 0 \\
\hline 01 & $\mathrm{X}$ & $X$ & 1 & 0 \\
\hline 10 & 0 & 1 & 0 & 1 \\
\hline 11 & 0 & 0 & 0 & $X$ \\
\hline
\end{tabular}

(b) A Decomposition Chart

Figure 2.2: A Kmap vs a Decomposition Chart

called a Non-Disjoint Decomposition, as shown in Figure 2.1(b). In Figure 2.1(b) $C \subset A$.

\subsection{Basic Notions and Definitions}

\subsubsection{A Karnaugh Map vs a Decomposition Chart}

Figure 2.2(a) shows a Karnaugh map representation of a function having four inputs and one output, Figure 2.2(b) shows the same function represented in a Decomposition chart. The only difference between a Karnaugh map and a Decomposition chart is that the row and column indices in a Karnaugh map are in Gray code while they are in binary order in a Decomposition chart. So from the Karnaugh map it can be seen that for an input variable combination of 1101 the output is " 0 ".

\subsubsection{A Minterm}

Definition 2.1 For a function $\mathbf{F}$ with $\mathbf{n}$ inputs, a minterm is a combination of values 0,1 of all its $\mathbf{n}$ argument values. 


\begin{tabular}{|c|c|c|c|c|}
\hline Row & $\mathrm{a}$ & $\mathrm{b}$ & $\mathrm{c}$ & $\mathrm{y}$ \\
\hline 1 & 1 & 0 & 1 & 1 \\
\hline 2 & 1 & - & 1 & 1 \\
\hline 3 & - & - & 1 & 1 \\
\hline 4 & 1 & 0 & 0 & 0 \\
\hline
\end{tabular}

Table 2.1: Table showing Minterms and Cubes

In the Table 2.1, row 1 is a minterm as none of the inputs are don't cares. In a Karnaugh map of a function, each cell in the Karnaugh map represents a minterm of the function.

\subsubsection{A Cube}

Definition 2.2 For a function $\mathbf{F}$ with $\mathbf{n}$ inputs, a cube is any combination of $\mathbf{n}$ in which, at least one of the inputs in $\mathbf{n}$ is a don't care.

In the Table 2.1, row 2 is a cube as input " $\mathrm{b}$ " is a don't care.

\subsubsection{Hamming Distance}

Definition 2.3 The Hamming distance of two minterms or cubes is the number of bits the two minterms or cubes differ in.

In Table 2.1 the minterm in row 4 and the minterm in row 1 have a Hamming distance of one, since they differ only in the value of input " $c$ ". In Table 2.1 the cube in row 2 and the minterm in row 4, also have a Hamming distance of one, because they have the same value for input "a", and for input " $b$ " row 2 has a don't care which means it can take any value, hence they only differ in the value of input variable "c".

Any two minterms, or any two cubes can be combined together in a Karnaugh map if they have a Hamming distance of one. 


\subsubsection{The Cofactor of a Function}

Definition 2.4 Any Boolean function can be represented by an expansion $f_{a}=$ $a f_{a}+\bar{a} f_{\bar{a}}$. This is called a Shannon Expansion. Here $f_{a}=\left.f\right|_{a=1}$ is called the positive cofactor of function $f$ with respect to variable $a$, and $f_{\bar{a}}=\left.f\right|_{a=0}$ is called the negative cofactor of function $f$ with respect to variable a.

Cofactors can be easily seen from a Karnaugh map, in Figure 2.2(a) column " 00 " represents the cofactor of the input function $f_{\bar{c}, \bar{d}}=\left.f\right|_{c=0, d=0}$, and row " 11 " represents the cofactor of the input function $f_{a, b}=\left.f\right|_{a=1, b=1}$. Hence the columns represent the cofactors with respect to the variables " $c$ " and " $\mathrm{d}$ ", and the rows represent the cofactors with respect to the variables "a" and " $b$ " in the Karnaugh map shown in Figure 2.2(a).

\subsubsection{The Free Set and the Bound Set}

As one of the first steps in Decomposition we split the input variables into two parts, the free set A and the bound set B.

Definition 2.5 In a Disjoint Decomposition, $F(A, B)=H(g(B), A)$ the free $\operatorname{set}(A)$ is the set of variables forming the rows of the Decomposition chart, and the bound set $(B)$ is the set of variables forming the columns of the Decomposition chart. In a Disjoint Decomposition $A \cap B=\emptyset$. In a Non Disjoint Decomposition $A \cap B \neq \emptyset$.

In Figure 2.2(b) variables $a, b$ are in the Free Set, and variables $c, d$ are in the Bound Set.

\subsubsection{Compatible and Incompatible Columns of a Decomposition Chart}

Definition 2.6 Two columns of a Decomposition chart are said to be compatible if they are the same or if assigning values to don't cares can make them the same. Compatibility between two columns is an incomplete tautology check between the two columns. 
In Figure 2.2(b) column 00 and column 10 are compatible. Column 00 and column 01 are not compatible because minterm (1000) is a " 0 " while minterm (1001) is a "1", hence the two columns are incompatible.

\subsubsection{Column Multiplicity}

Definition 2.7 The Column Multiplicity(v) of a Function is the number of incompatible columns in the Decomposition chart representing the function.

In Figure 2.2(b) column 00 is compatible with column 10 and columns 01 and 11 are compatible, hence the column multiplicity is " 2 ".

\subsubsection{A Compatibility graph vs an Incompatibility graph}

Figure 2.3 shows the columns of a Karnaugh map represented, as an Incompatibility Graph, in Figure 2.3(b), and as a Compatibility Graph in Figure 2.3(c). Nodes represent columns in the Karnaugh map labeled as 1,2,3,4 in Figure 2.3(a).

Definition 2.8 In an incompatibility graph an edge between two nodes, where the nodes represent columns of a Karnaugh map, means that the two columns are incompatible. In a compatibility graph an edge between two nodes means that the two columns are compatible.

In the Karnaugh map shown in Figure 2.3(a), because column 1 has all don't cares, it has no edges in the incompatibility graph shown in Figure 2.3(b), and edges with all the other nodes in the compatibility graph shown in Figure 2.3(c).

\subsubsection{Ashenhurst and Curtis Decompositions}

The two main Decompositions that will be discussed here are Ashenhurst [1] and Curtis Decomposition [2]. Figure 2.4 shows an Ashenhurst Decomposition. The basic requirement for an Ashenhurst Decomposition is that the $\mathrm{G}$ block can have at most one output. Disjoint Ashenhurst Decompositions occur rarely for completely specified binary functions. Figure 2.5 shows a Curtis Decomposition, 


\begin{tabular}{c|c|c|c|c|}
1 & 2 & 3 & 4 \\
0 & $x$ & 0 & 0 & 1 \\
\cline { 2 - 5 } 01 & $x$ & $x$ & 0 & 1 \\
\hline 11 & $x$ & 0 & $x$ & 0 \\
\hline 10 & $x$ & 1 & 1 & 0 \\
\hline
\end{tabular}

(a) A Karnaugh Map of Function F

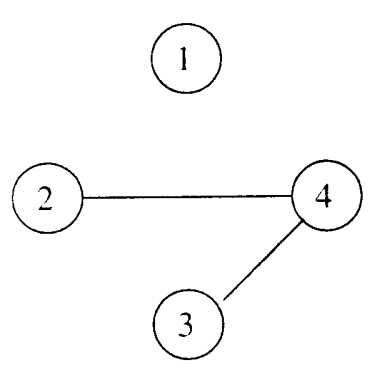

(b) Incompatibility Graph of Function $\mathrm{F}$

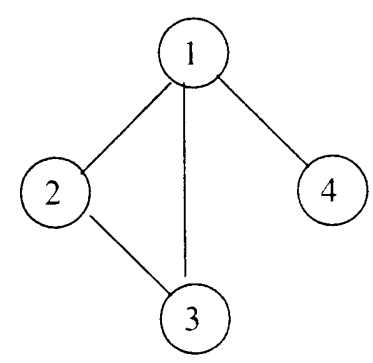

(c) Compatibility Graph of Function $\mathrm{F}$

Figure 2.3: A Compatibility graph and an Incompatibility graph of a Function

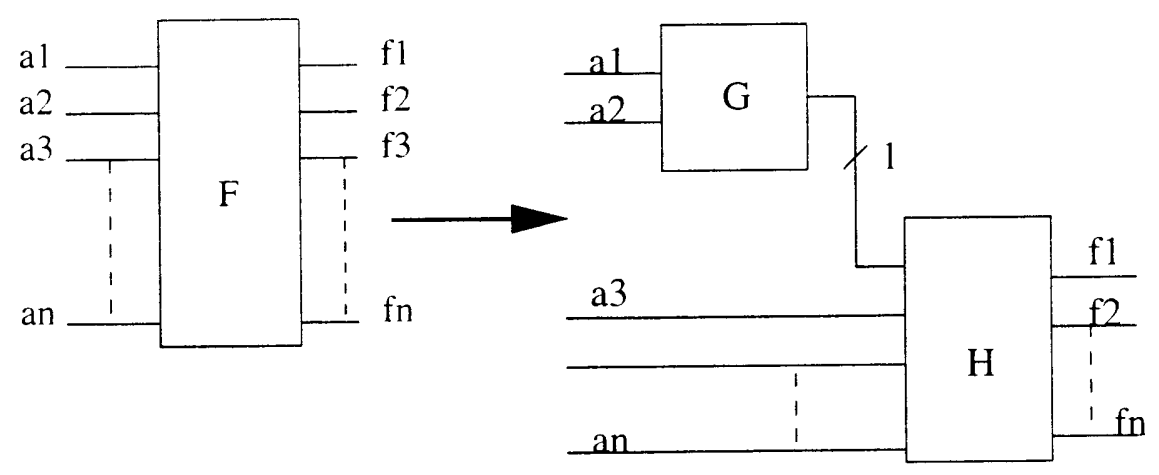

Figure 2.4: An Ashenhurst Decomposition 


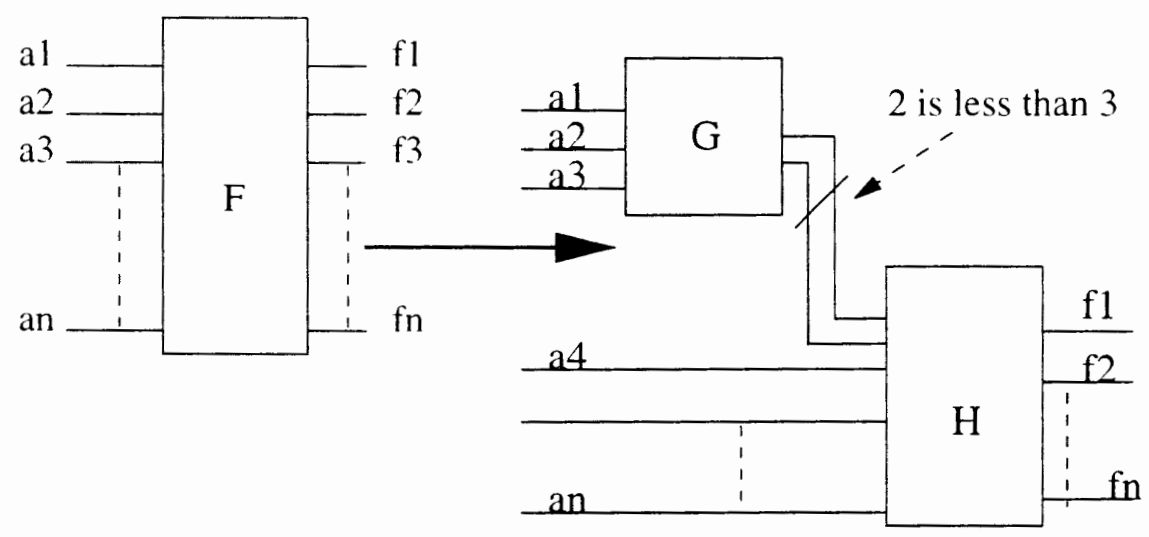

Figure 2.5: A Curtis Decomposition

Curtis Decomposition occurs very often. For a Curtis Decomposition to exist, the block $\mathrm{G}$ must have less outputs than inputs.

\subsection{The Steps involved in performing a Curtis Decomposition}

Now the steps involved in performing a Curtis Decomposition will be explained with the help of an example.

Figure 2.6 shows how the Curtis Decomposition process is performed on a binary function. The aim here is to decompose the function $\mathrm{F}$ into two functions which we call "G" and " $H$ ". The inputs to function " $G$ " will be the variables in the bound set, and the inputs to the function " $\mathrm{H}$ " will be the free set variables and the outputs of the "G" function. As the first step to decomposition a bound set is selected. This is the Variable Partitioning Step. Figure 2.6(a) shows the Decomposition chart of a function $F$ which has five inputs and one output. As can be seen from this Figure, the bound variables have been chosen $a \mathrm{~s} a, b$ and $c$, and the free variables are $d$ and e. As the next step the columns which are compatible have to be grouped together. For this step either a Compatibility or an Incompatibility Graph can be created. In this example an Incompatibility Graph as shown in Figure 2.6(b) is created. The Incompatibility Graph is a direct mapping of the columns of the Decomposition chart, the number of nodes in the 


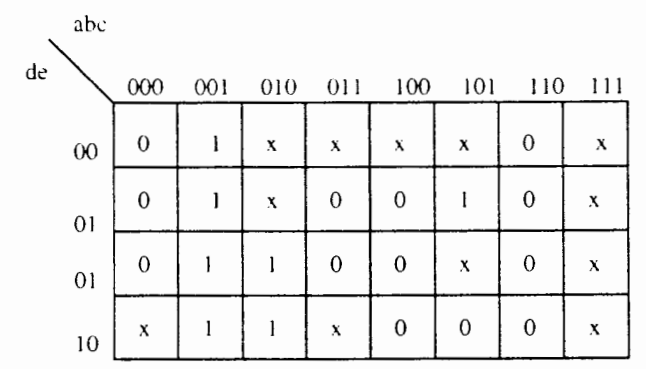

(a ) Decomposition Chart of Function $\mathrm{F}$

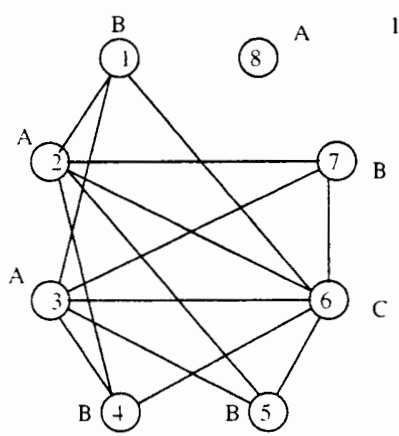

(b) Incompatibility Graph of Function $\mathrm{F}$

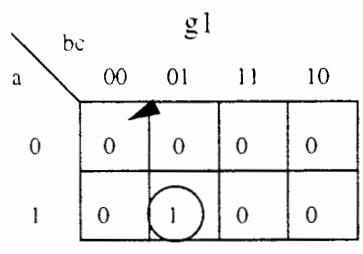

$-$

$$
\mathrm{g} 1=\mathrm{abc}
$$

(d) Karnaugh map of $g 1$
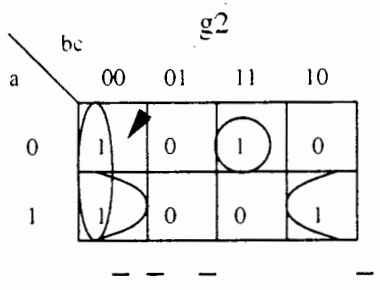

$\mathrm{g} 2=\mathrm{bc}+\mathrm{abc}+\mathrm{ac}$

(e) Karnaugh map of g2

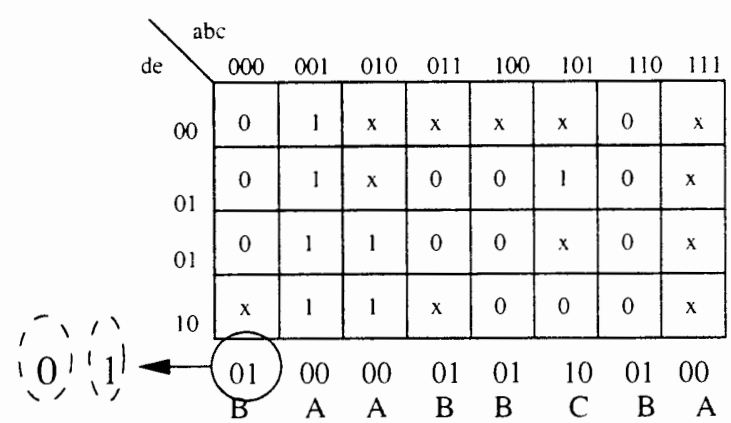

(c) Decomposition Chart after Graph Coloring showing Encoding

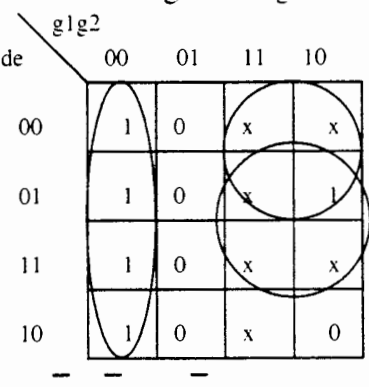

$\mathrm{H}=\mathrm{g} 1 \mathrm{~g} 2+\mathrm{d} \mathrm{g} 1+\mathrm{e} \mathrm{g} 1$

(f) Karnaugh map of $\mathrm{H}$

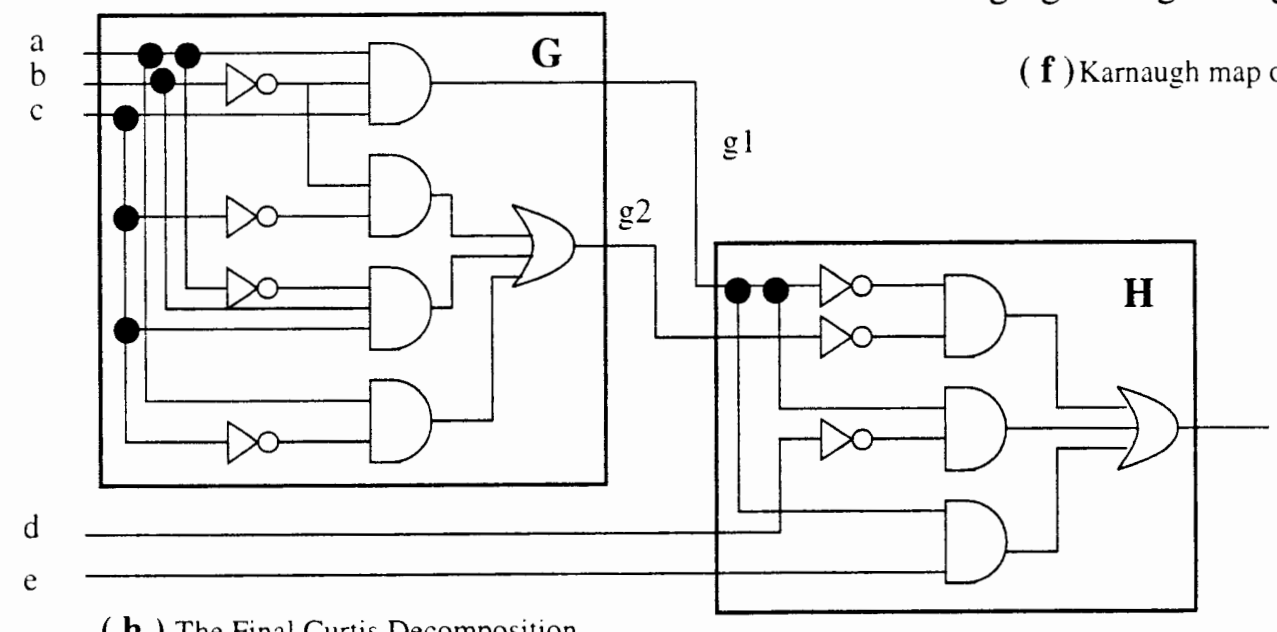

( h ) The Final Curtis Decomposition

Figure 2.6: The Steps involved in Performing a Curtis Decomposition 
Incompatibility Graph is equal to the number of columns in the Decomposition chart. The Graph Coloring technique is used to color the graph, and thus find out which columns are compatible. Graph coloring will be explained in more detail in Chapter 3. After assigning the colors it is found that there are three colors involved ( colors are represented by letters in Figure 2.6(b)). Figure 2.6(b) shows the colored graph. Figure 2.6(c) shows the columns with the colors assigned to them.

Now the encoding step is done. Since two bits are required to encode the three colors ( as found from the graph coloring), this tells us that there are two outputs from the $\mathrm{G}$ block. These are intermediate variables which we call $g_{1}$ and $g_{2}$. Figure 2.6(c) shows the encodings chosen here, in an arbitrary way. Columns with color A are given the code "00", columns with color B are given the code "01", and columns with color $\mathrm{C}$ are given the code " 10 ". The Karnaugh maps representing the Functions $g_{1}$ and $g_{2}$ are shown in Figure 2.6(d) and Figure 2.6(e), respectively. We know that the inputs to the $\mathrm{G}$ Block are variables $\mathrm{a}, \mathrm{b}$ and $\mathrm{c}$, hence these variables form the indices of the Karnaugh maps of $g_{1}$ and $g_{2}$. In order to make the Karnaugh map of function $g_{1}$, for abc $=000$ ", it is seen in Figure 2.6(c) that the encoding given to that column is " 01 ", so the first bit, bit " 0 " is put in the Karnaugh map of function $g_{1}$ at location " 000 " in Figure 2.6(d), and the second bit, bit " 1 " is put in the Karnaugh map of function $g_{2}$ at location "000" in Figure 2.6(e). In this way the Karnaugh maps of functions $g_{1}$ and $g_{2}$ are created. The " $H$ " function is shown in Figure 2.6(f). Variables " $\mathrm{d}$ " and "e" of the free set, and the outputs of the G block $g_{1}$ and $g_{2}$ form the indices of this Karnaugh map. For column with $g_{1}, g_{2}=$ "00" which is colored with A, all the columns in the decomposition chart shown in Figure 2.6(c) having color A are combined together to form one column which is included in all the columns with color A. In order to form this column don't cares can get assigned values. This finally results in a column with all four 1's, which forms column " 00 " in Figure 2.6(f). In this way the Karnaugh map of the $\mathrm{H}$ function is created. Then the Karnaugh maps of functions $g_{1}, g_{2}$ and $\mathrm{H}$ are solved and the logic required to 
implement the $\mathrm{G}$ and $\mathrm{H}$ blocks is obtained. Figure 2.6(g) shows the final Curtis Decomposition with the logic required to implement the $\mathrm{G}$ and $\mathrm{H}$ blocks.

\subsection{Applications of Functional Decomposition}

Functional Decomposition is an NP-Complete Problem, and due to this complexity it is not very commonly used in the industry, though recently there has been a renewed interest in Decomposition. In TRADE [5] Functional Decomposition provides very good results for mapping a function into a Xilinx Field Programmable Gate Array(FPGA). Functional Decomposition can be used for Look up table (LUT) based FPGAs to minimize the number of LUTs used. Decomposition can also be used for VLSI design and other FPGAs such as Fine Grain FPGAs, Atmel, Motorola etc and for complex Programmable Logic Devices (PLD's). Decomposition can also be used in PLA Decomposition. Decomposition is also used in areas like image processing, machine learning, knowledge discovery, knowledge acquisition, database optimization, AI, image coding, automatic theorem proving and verification of software and hardware. In Machine learning, Decomposition can be used to extract the pattern in a function. This pattern is basically the reduced decomposed function. Decomposition of relations which will be introduced in Chapter 6, finds applications in areas like state assignment of non-deterministic state machines, Machine Learning and Knowledge Discovery from databases. Thus we conclude this Chapter by stating that Decomposition is a very powerful tool, but it still has to attain its full potential in the industry. 


\section{CHAPTER 3}

\section{SOME NEW APPROACHES TO GRAPH COLORING: TWO PROGRAMS AND TWO IDEAS}

Finding the Column Multiplicity for a chosen bound set is very important for the success of a Functional Decomposer program, and a high percentage of the run time of the Functional Decomposer is spent on the Column Minimization part of Decomposition. What is needed is not only a fast method to solve the problem, but also one which produces results as close to optimal as possible.

Here we want to find out what is the role of Column Minimization in the overall success of a Decomposer; especially, in terms of the calculation time, the memory usage, and the quality of results. We want to investigate how the answers to these questions depend on the type of data, for instance on the percent of don't cares, or on the density of graphs in question. Presently the decomposer introduced by Pedram et al [17] achieves the best results; this program uses a set covering approach. But this decomposer is primarily for circuit applications. It is commonly believed that the primary success of this decomposer is due to the good data structures used. It is believed that reformulating the problem from a set covering approach to a graph coloring approach may significantly improve the efficiency of the decomposition. Graph Coloring for decomposition was introduced by Muzio and Wesselkamper [20] and Perkowski [13]. It is not a new idea in logic synthesis but is often overlooked as an alternative approach to Set Covering but is actually a more suitable choice in many problems. Recently there are more approaches that use Graph Coloring [21]

There are basically four methods to find the Column Multiplicity in Functional Decomposition, namely Set Covering, Graph Coloring, Clique Partitioning and 
Clique Covering. A relation between the columns of the Karnaugh map of a function, for a given bound and free sets can be represented as a Compatibility Graph or as an Incompatibility Graph. If represented as a Compatibility Graph, nodes which are connected together are compatible nodes and can be colored with the same color. This is called Clique Covering. If the graph is represented as an Incompatibility Graph then nodes which do not have a common edge can be colored with the same color. This is called Graph Coloring. Even though there has been a lot of research done in the field of Graph Coloring and Functional Decomposition, no one, to our knowledge, has compared these methods, or evaluated the importance of finding minimal solutions to the problem of Column Multiplicity in the Ashenhurst/Curtis Decompositions.

In this Chapter two new approaches to Graph Coloring will be introduced. One of the Graph Coloring methods is a heuristic algorithm and the other is an Exact Graph Coloring. The heuristic Algorithm(DOM), which uses dominations to color the graph was presented in [18] by Perkowski. The idea for the Exact Graph Coloring Algorithm(EXOC) was a modification of an idea given to me by Perkowski. Also in this Chapter ideas for two new exact Graph Coloring algorithms are presented. The first idea extends the heuristic Graph Coloring algorithm to an exact Graph Coloring algorithm using dominations (EXDOM). The second idea combines the heuristic Graph Coloring algorithm and the exact Graph Coloring algorithm to an exact Graph Coloring algorithm. Before introducing the Graph Coloring algorithms and programs, some basic notions and definitions are presented.

\subsection{Basic Notions and Definitions}

\subsubsection{Dominations in an Incompatibility Graph}

Definition 3.1 Some node " $A$ " in an incompatibility graph dominates some other node " $B$ " in the graph if the following is satisfied:

1) Node " $A$ " and node " $B$ " have no common edge. 


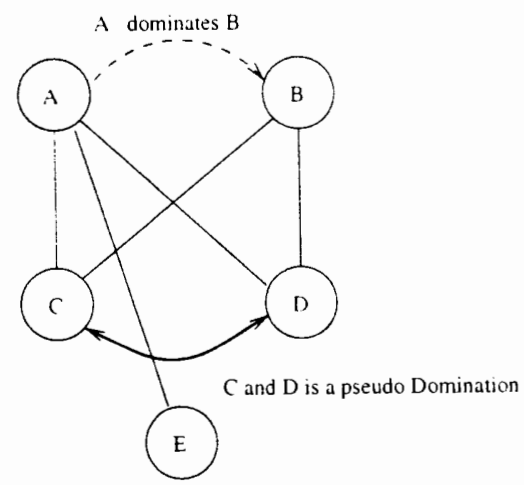

Figure 3.1: Dominations and Pseudo Dominations in an Incompatibility Graph

2) Node " $A$ " has edges with all the nodes that node " $B$ " has edges with.

3) Node " $A$ " has at least one more edge than node " $B$ ".

In Figure 3.1, node "A" dominates node " $\mathrm{B}$ " since it satisfies the conditions for domination. When two nodes have a domination then both the nodes can be colored with the same color.

Definition 3.2 If conditions 1) and 2) for dominations is satisfied and node " $A$ " has the same number of edges as node " $B$ ", then it is called a pseudo domination.

In Figure 3.1, nodes $\mathrm{C}$ and $\mathrm{D}$, have a pseudo domination.

Theorem 3.1 If any node " $A$ " in a graph dominates any other node " $B$ " in the graph, node " $B$ " can be removed from the graph, and in a pseudo domination any one of the nodes " $A$ " or " $B$ " can be removed.

Proof 3.1 The proof for the Theorem 3.1 is that if any node " $A$ " in a graph dominates any other node " $B$ " in the graph, it means that node " $B$ " is a proper subset of node " $A$ " hence by removing node " $B$ " we are not changing the number of colors in the graph, as node " $A$ " holds all the edge information that node " $B$ " holds. 


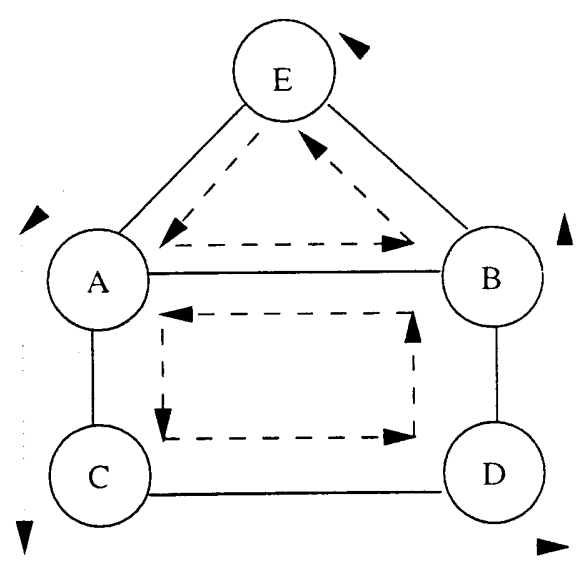

Figure 3.2: A Cyclic Graph

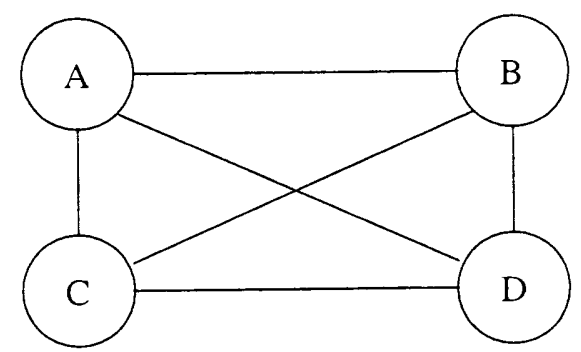

Figure 3.3: A Complete Graph

\subsubsection{A Cyclic Graph and Cycles in an Incompatibility Graph}

Definition 3.3 In a cyclic graph no dominations can be found, but pseudo dominations may be found.

Definition 3.4 A Cycle in an Incompatibility Graph is a circuit that passes through every vertex exactly once.

In Figure 3.2 circuit consisting of vertices $\mathrm{E}, \mathrm{A}$ and $\mathrm{B}$ is a cycle.

\subsubsection{A Complete Graph}

Definition 3.5 A Complete graph [15] is one in which all vertice pairs are connected. 
In a complete graph

$$
\text { total_edges }=\frac{\text { nodes } *(\text { nodes }-1)}{2}
$$

where total_edges is the sum of all the edges in the graph. In a Complete Graph no dominations or pseudo dominations can be found. In a Complete Graph all the nodes must have a unique color. A Complete Graph is a special case of a Cyclic Graph. Figure 3.3 shows an example of a Complete Graph.

\subsection{A New Approach to a Heuristic Graph Coloring Using Domina- tions}

In this section a new heuristic algorithm which uses the concept of dominations(explained in Section 3.1.1) to color an incompatibility graph is presented. This heuristic graph coloring algorithm has been given the name $D O M$, and in future sections it will be referred to by this name. In order to properly introduce $D O M$, first some examples which show how DOM colors an incompatibility graph are presented, and then the algorithm and implementation details of DOM are given.

\subsubsection{An Example showing how DOM colors a non cyclic graph}

The following steps explain how DOM colors a non cyclic graph.

1. Figure 3.4(a) shows an Incompatibility Graph. As can be seen Node 2 is dominated by Node 1, so in Figure 3.4(b) Node 2 is removed and it is remembered that it was dominated by Node 1 . The proof that a dominated node can be removed from the incompatibility graph was given in Section 3.1.1.

2. Next, in Figure 3.4(b) Node 5 is removed as it is dominated by Node 4, and it is remembered that Node 4 dominates Node 5. 


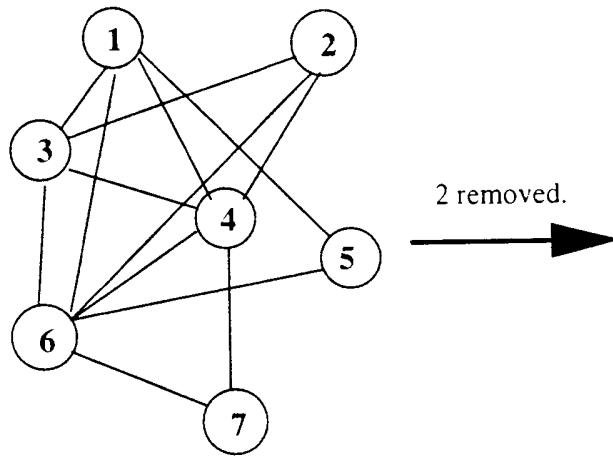

(a) 1 dominates 2

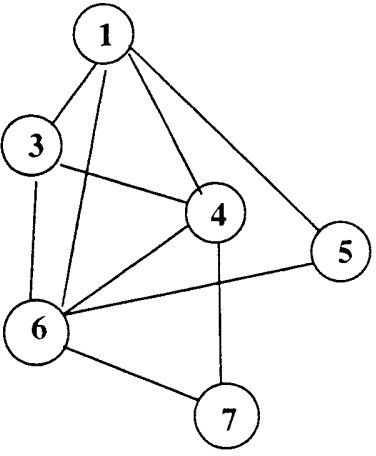

(b) 4 dominates 5

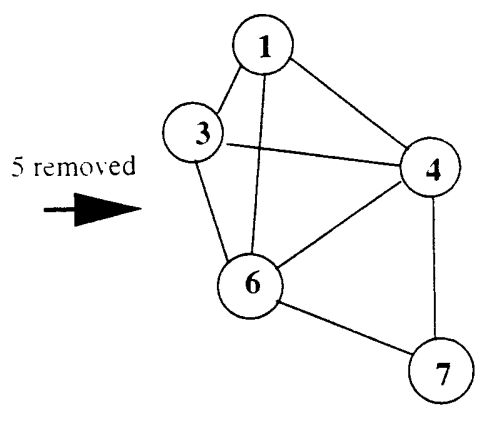

(c) 1 dominates 7

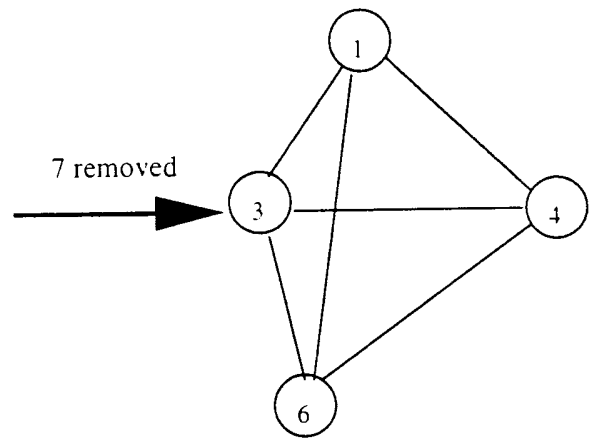

(d) Complete Graph

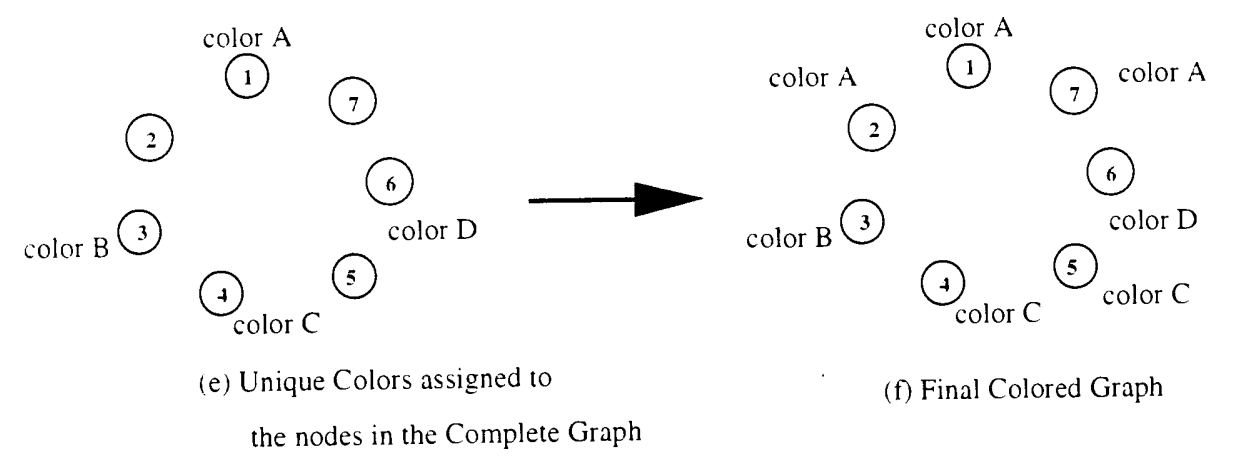

Figure 3.4: Example showing how DOM colors a non cyclic graph 
3. Then in Figure 3.4(c), it can be seen that Node 7 is dominated by both nodes 1 and 3 , the choice made is the first node which is Node 1 , and Node 7 is removed. It is now remembered that Node 1 now dominates Node 2 and Node 7.

4. After removing Node 7 the resulting graph shown in Figure 3.4(d) is a complete graph, so go to Step 5. In a complete graph(shown in Section 3.1.3), each node is connected to all the other nodes, each node in the complete graph must have a unique color.

5. In Figure 3.4(e), each node in the Complete Graph is given a unique color.

6. Finally in the last step in Figure 3.4(f) the dominated nodes are colored with the same color as the dominating node. The color assignments are Color A $\{1,2,7\}$.

Color B $\{3\}$.

Color C $\{4,5\}$.

Color D $\{6\}$.

Thus in this way the graph is colored. Figure 3.4(e) shows the completely colored graph. Four colors were used which is the minimum required for this graph.

\subsubsection{An Example showing how DOM colors a cyclic Graph}

This Example illustrates how DOM colors a cyclic graph.

1. An incompatibility graph is shown in Figure 3.5(a), as can be seen this graph has a number of cycles.

2. As the first step the graph is checked for dominations, but no dominations are found in this graph, so the first node is removed from the graph, which is node 1 , and it is assigned a minimum possible color which in this case is color A. 


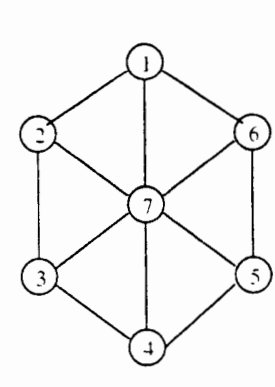

(a)<smiles>[O]</smiles>

(2)<smiles>o1oo1</smiles>

(c)

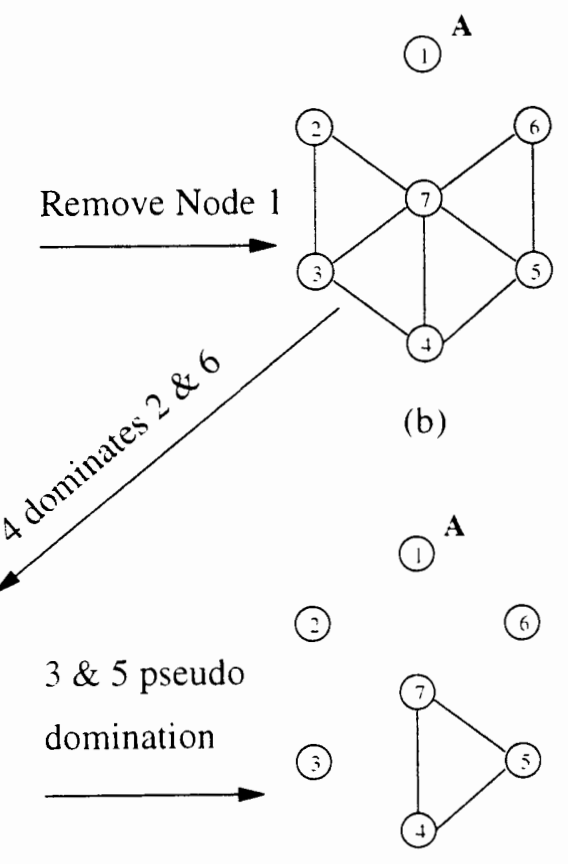

(d)

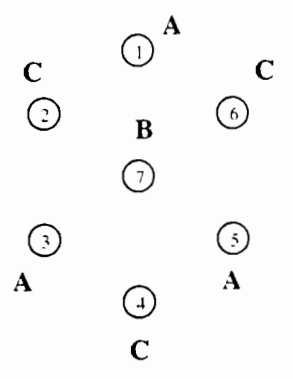

(e)

Figure 3.5: Example showing how $D O M$ colors a cyclic graph 
3. This results in a new graph, shown in Figure 3.5(b). In this graph node 4 dominates node 2 and node 6 . So node 2 and node 6 are removed from the graph, and it is remembered that node 4 dominates node 2 and node 6.

4. On removing node 2 and node 6 , in the resulting graph shown in Figure 3.5 (c) nodes 3 and 5 have a pseudo domination so the first one of these nodes which is node 3 is removed, and then node 4, 5, and 7 form a complete graph. The complete graph is shown in Figure 3.5(d).

5. Now nodes are colored with the minimum possible color, and each dominated node is given the same color as the node which dominated it. The coloring is shown in Figure 3.5(e). Three colors were used to color the graph, which is the minimum required for this graph. The color assignments are:

Color $\mathrm{A}\{1,3,5\}$.

Color $B\{7\}$.

Color C $\{2,4,6\}$.

\subsubsection{Different Graphs Possible in Decomposition, and how they are handled by $D O M$}

Figure 3.6 shows some possible classes of graphs, that might be generated during the different stages of Functional Decomposition, and the colorings that $D O M$ would find for these graphs. The heuristic assumption of our approximate algorithms will be that all graphs will fall into one of these classes, only they will have different number of nodes and different number of edges. Figure 3.6(a) shows a Complete Graph, in this case DOM will generate a unique color for each node in the Complete Graph. Figure 3.6(b) shows a cyclic graph with dominations, in this case $D O M$ will find that node 3 dominates node 2 , and node 1 dominates node 4. Then $D O M$ will remove the dominated nodes 2 and 4 , and the resultant graph is a complete graph. Then each node in the complete graph is given a unique color and the dominating nodes are colored the same color as the nodes which 


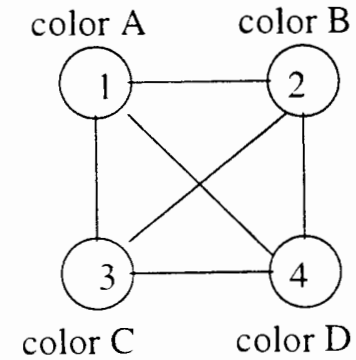

( a ) A Complete Graph

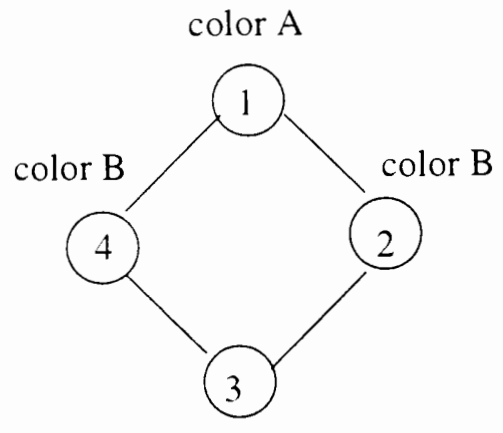

color A

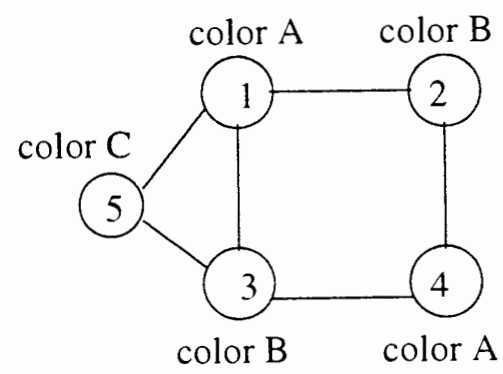

(b) A Cyclic Graph with Dominations

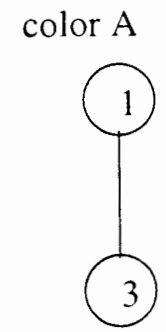

color B color A

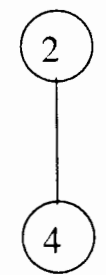

color B

(c) A Cyclic Graph with

(d) A Disjoint Graph

Pseudo Dominations.

Figure 3.6: The Different Classes of Incompatibility Graphs that may be generated in the steps of Functional Decomposition 
dominate them. Figure 3.6(c) shows a cyclic graph with pseudo dominations, here $D O M$ will find that nodes 1 and 3 have a pseudo domination and will give them the same color, and nodes 2 and 4 have a pseudo domination and will give them the same color. Figure 3.6(d) shows a disjoint graph, here DOM will choose one of the nodes, assign it a minimum possible color, and then remove it from the graph and go back to checking for dominations. As can be seen in all the cases shown, DOM will generate the minimum solution.

\subsubsection{Implementation Details of DOM}

The Algorithm for DOM has been divided into two parts:

1. Function check-dominations which checks for dominations in the graph, it has two possible return values.

(i) Returns "TRUE" if the graph was complete.

(ii) Returns "FALSE" if the graph was cyclic, or disjoint.

2. Function graph_coloring is the top level function, which calls check_dominations.

\subsubsection{Pseudo Code for Function graph_coloring}

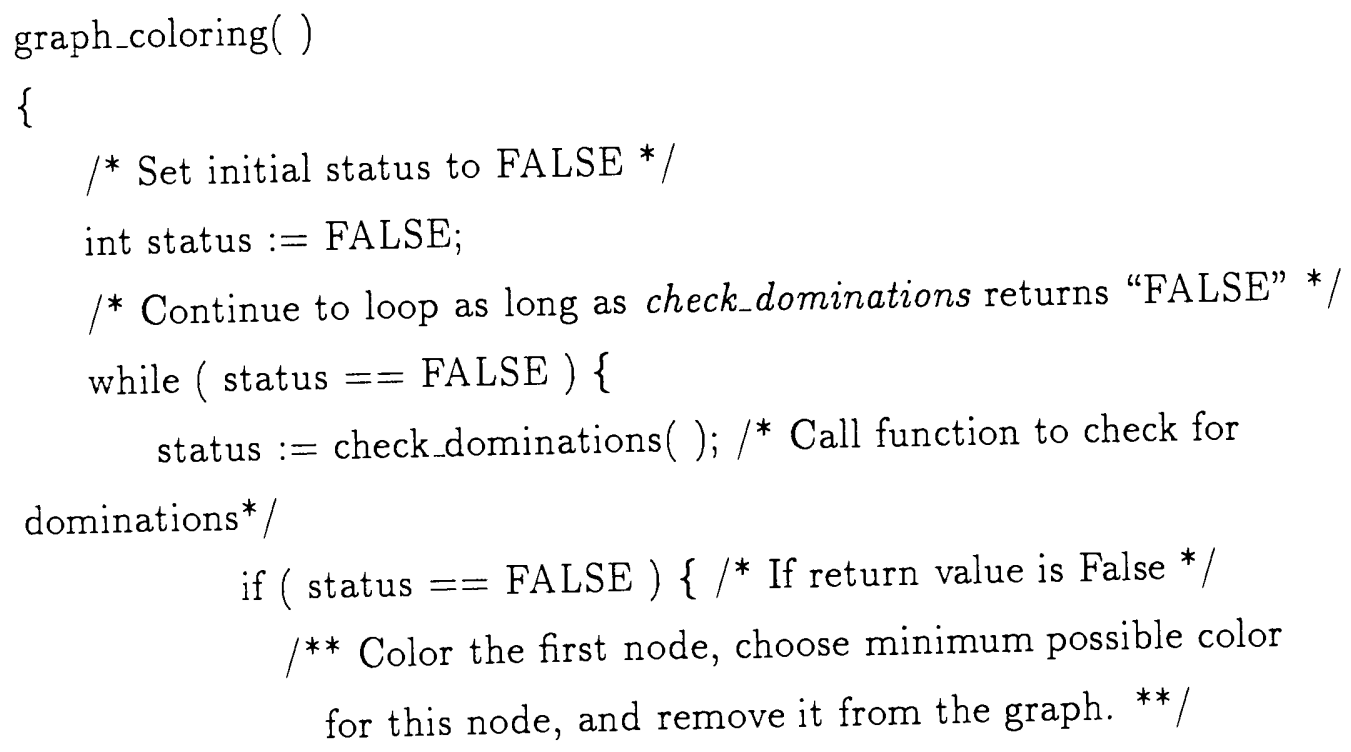




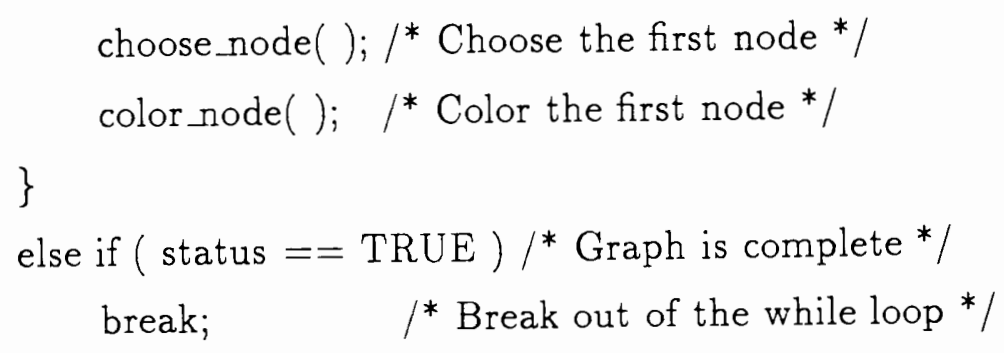

\subsubsection{Algorithm for Function check_dominations()}

Step 1: Choose the node with the most incident edges, if tie choose the first one.

Step 2: If the graph is complete, then, return with value TRUE.

Step 3: Check for dominations of the chosen node with all the other nodes.

Step 4: If a domination is found, then, store the information of the dominating node, and the node that it dominates.

Step 5: Remove the edges of the dominated node from the incompatibility graph.

Step 6: If the chosen node has not been checked with all the other nodes go to Step 2 , else go to Step 7.

Step 7: If all the nodes have been checked, return with value FALSE, else go to Step 1.

\subsubsection{Pseudo Code for Function check_dominations() check_dominations()}


\{

int dominating, dominated;

/* Loop for the number of nodes */

for $(\mathrm{i}=1 ; \mathrm{i} \leq$ number_of_nodes; $\mathrm{i}++)\{$

if ( complete_graph ()$) / *$ Function checks if graph is complete */ return TRUE;

dominating $=$ select_a_node ()$; / *$ Choose a node $* /$

$/^{*}$ Loop for all the nodes $-1 * /$

for ( dominated $=1$; dominated $\leq$ number_of_nodes -1 ; dominated ++ ) \{

if (domination found(dominating, dominated) $)\{$

/* Store the information of dominating and dominated nodes */ mark_dominating_node( dominating, dominated );

$/ *$ Remove the edges of the dominated node */

remove_edges(dominated );

\}

\}

\}

${ }^{*}$ If we land here it means we were unable to find dominations */

return FALSE;

\}

\subsubsection{The strong and weak points of $D O M$}

By the examples shown in Figure 3.4 and Figure 3.5 we showed cases when $D O M$ will generate the best solution. We do not claim that $D O M$ will generate the best solution for all possible graphs, but we think it will perform well in most cases.

One weak point of the algorithm is when no dominations are found, then a node is selected and assigned a minimum possible color. If the coloring of this node is a bad choice ( a bad choice is one which will lead to a non minimal coloring), it will result in a solution which is not minimal. Another weak point of the algorithm 
is when it fails to find dominations or pseudo dominations at any stage of the coloring. In a graph like this, since DOM will choose a node and color it at each stage, it is basically doing a greedy coloring, and a greedy algorithm will get the same solution in a much faster time. But we think that these kind of complicated graphs(worst case graphs) will rarely, if ever, occur during the steps of Functional Decomposition, which is the application in which we are interested.

The strong point of $D O M$ is that it can find the minimum solution without backtracking in all the cases when the graph which results after checking for dominations is a complete graph. Thus this program will be effective in finding the minimum solution in all non-cyclic graphs. The program may not be able to find the best solution in a cyclic graph. 


\subsection{An idea which extends $D O M$ to an Exact Graph Coloring}

In this Section an idea of how the algorithm for $D O M$ can be made into an algorithm, which will always generate the minimum number of colors for an incompatibility graph is presented. This new algorithm will be called EXDOM. In the previous section it was shown that $D O M$ will always generate the exact solution whenever the resulting graph at any stage of checking for dominations is complete, it is only when the graph is cyclic that DOM may not generate the exact solution. On coming across such a graph, a tree search is done in order to make DOM exact. This tree search is explained with the help of an example.

The tree search method shown in Figure 3.7 will be used by EXDOM whenever the function check_dominations (this algorithm was explained in Section 3.2.4.3) which checks for dominations in the graph has a return value of FALSE. Figure 3.7 shows how EXDOM colors a cyclic graph.

1. The block labeled SNODE 1 in Figure 3.7 is a cyclic graph which has 6 nodes in the cycle (EXDOM has no knowledge about the nature of the cyclic nodes, that is how many cycles are there). So the information of which nodes are in the cycle, is stored.

Select node 1. Assign the minimum possible color to node 1 (The minimum possible color is the first possible color that can be assigned to a node which does not conflict with any nodes already colored).

Color chosen is A. Remove the edges of node 1.

2. Removing Node 1 results in a new graph, shown in block labeled SNODE2 a). In this graph the function check_dominations returns a value of TRUE, since it finds a complete graph. The steps of checking for dominations are shown in block labeled SNODE 2 in Figure 3.7. So in SNODE 2 a solution is obtained, which uses 3 colors to color the graph. The color assignment is:

Color A: $\{1,3,5\}$,

Color B: $\{2,4\}$, 


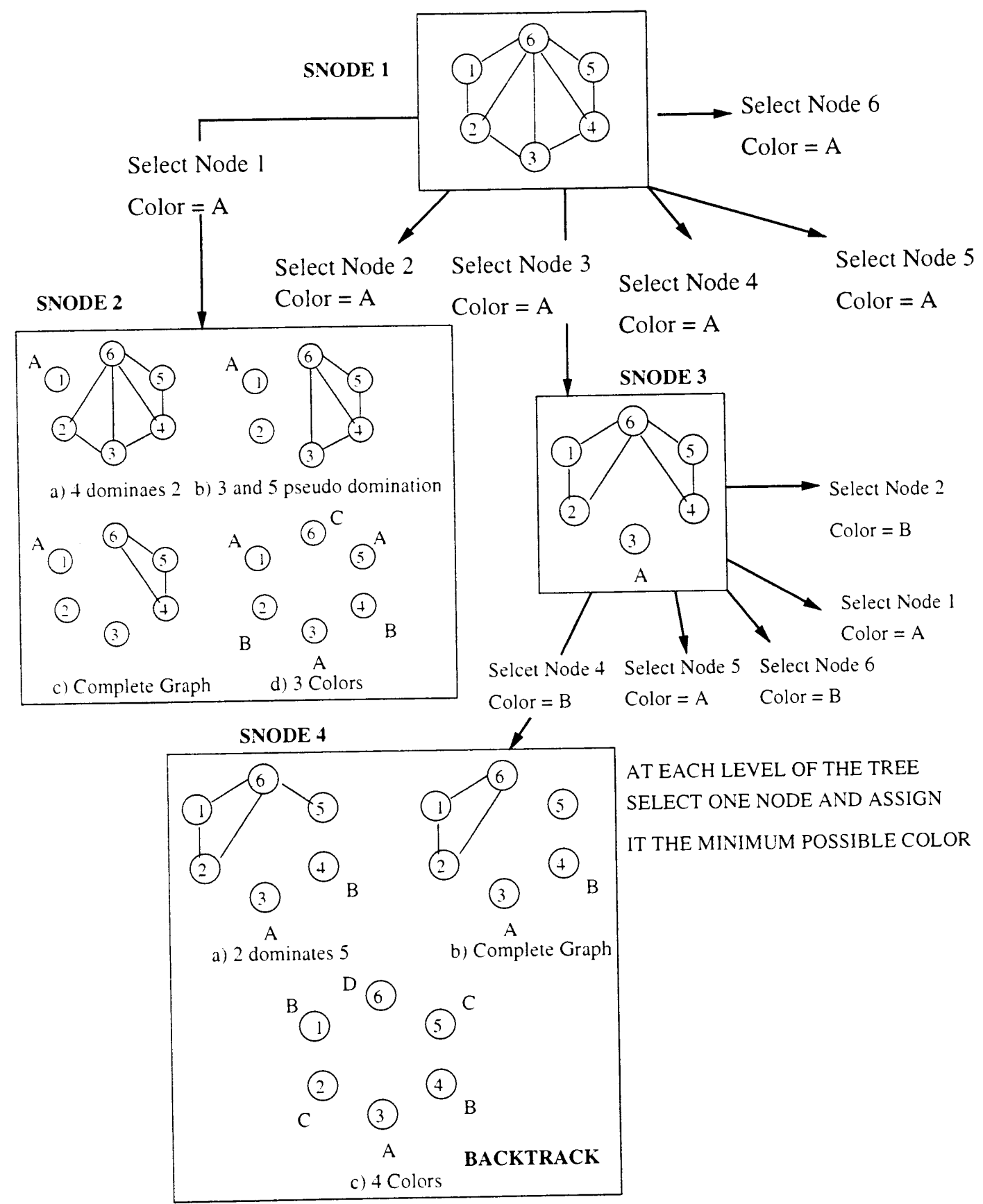

Figure 3.7: The tree Search showing how DOM can be made exact when it encounters a cyclic graph 
Color C: $\{6\}$.

Backtrack to SNODE 1.

3. Now in SNODE 1, a new node in the graph which has not been selected before is selected. In any SNODE nodes are selected in a sequential order, until all the nodes in that SNODE have been selected. The next node selected in SNODE 1 will be Node 2, but this is not shown in the Figure 3.7. Instead, the next path of the tree, which is selecting Node 3 in SNODE 1 is shown.

4. On selecting Node 3 in SNODE 1 and giving it color A, a new graph is arrived at, shown in SNODE 3. This graph is still a cyclic graph. So select the first node in this graph, which is Node 4.

Node 4 is assigned the minimum possible color which is color B. So in SNODE 4 the coloring led to four colors. The color assignment is:

Color A: $\{3\}$,

Color B: $\{1,4\}$,

Color C: $\{2,5\}$,

Color D: $\{6\}$.

Since this solution is worse the the previous solution, discard this solution. Backtrack to SNODE 3.

5. In SNODE 3 the next node choice is Node 5. So go along this new tree path, and repeat for all the nodes in SNODE 3. Repeat this process for all the nodes in all the SNODEs(for which check_dominations returned FALSE), at every level of the tree. If at any stage a better solution is found save the new solution and discard the old solution. For simplification, Figure 3.7 does not show the entire search space.

\subsubsection{Algorithm for EXDOM}

The Algorithm for EXDOM has been divided into two parts: 
1. Function check_dominations which checks for dominations in the graph, it has two possible return values.

(i) Returns "TRUE" if the graph was complete.

(ii) Returns "FALSE" if the graph was cyclic, or disjoint.

2. Function graph_coloring is the top level function, which calls the function check_dominations.

Function check_dominations is the same as in DOM which was explained in Section 3.2.4.3. The following is the Algorithm for the function graph_coloring.

\subsubsection{Algorithm for Function graph_coloring for EXDOM}

NODES is the set of nodes.

NODE_COUNT is the count of the nodes in the graph.

$D O M_{-} C O U N T$ is the count of the dominated nodes.

$C Y C_{-} N O D E S$ is the number of nodes in the cycle.

$C Y C_{-} N O D E S$ is equal to NODE_COUNT-DOM_COUNT.

$S N \quad$ is the selected node.

$B E S T \_S O L N$ is the number of colors assigned, initially set to be equal to NODE_COUNT

$N E W_{-} S O L N \quad$ is the new number of colors.

\section{Step 1. Initial Check to see if depth first search needed:}

Call function check_dominations to check for dominations. Every time function check_dominations finds a domination the dominated node is removed from the graph, and the count of dominated nodes removed ( DOM_COUNT) is incremented. check_dominations returns TRUE if graph was complete, or FALSE if graph was not complete. if $($ check_dominations $==\mathrm{TRUE}) /{ }^{*}$ If return value is TRUE $* /$ 
store the solution and exit.

else

go to Step 2.

Step 2. Creating the Initial State:

Find $C Y C_{-} N O D E S=N O D E_{\_} C O U N T-D O M_{-} C O U N T$

$S N=C Y C \_N O D E S[0] \quad$ Select one node from cyclic nodes.

Choose minimum possible color for $S N$.

Remove $S N$ from the graph.

$C Y C_{-} N O D E S=C Y C \_N O D E S-1 /{ }^{*}$ Decrement count of cyclic nodes*/

Go to Step 3.

Step 3. If $\left(C Y C_{-} N O D E S \neq \emptyset\right)$

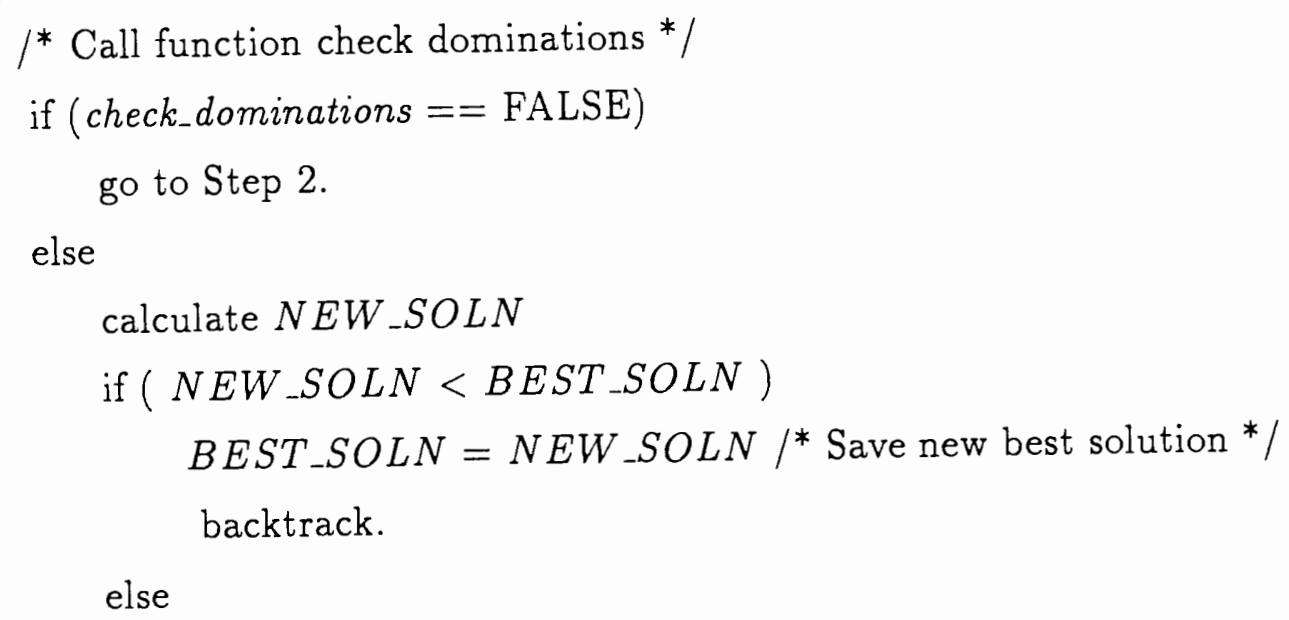

backtrack.

Theorem 3.2 The search strategy shown for EXDOM in Section 3.3 will always be able to generate the exact solution.

Proof 3.2 This search strategy is a depth first search with one child, which means that for any node of the tree only one child is generated at a time, and each child of a parent node is deleted on returning to the parent of the node. At each level of the tree a different node in the cycle at that level of the tree is selected, and it is assigned the minimum possible color. Thus on reaching the leaf nodes of 
the tree all the possible solutions for the graph will be arrived at. The best of these solutions will be the chromatic number of the graph being colored. Thus this proves Theorem 3.2

By this search many best solutions for the graph may be arrived at but only the first best solution is saved. This method has not been programmed yet, hence there are no results for the method. 


\subsection{A New approach to an Exact Graph Coloring}

Since Graph Coloring is an NP Complete problem, in general nearly all the possible solutions have to be evaluated, in order to find the best solution. The algorithm used here for the exact Graph Coloring is a greedy algorithm with backtracking and cut-off. This will be explained in more detail in this section.

Here a new algorithm for coloring an incompatibility graph, with the minimum number of colors possible for this graph is presented. This algorithm is called $E X O C$, which stands for Exact One Child. In all latter sections the algorithm will be referred to by this name. EXOC uses a tree search in order to color the graph, and colors successively nodes with an actually available color of a smallest number, remembering for each node all the remaining possibilities of coloring (it is assumed that initially the set of colors has as many elements as the set of nodes).

Definition 3.6 The chromatic number of a graph is defined as the number of colors in the exact solution.

EXOC uses a "depth-first with one child" strategy. In this strategy at every stage only one branch of the tree is generated, and after finding a solution in a new branch, the solution that is better than a previous one, EXOC has a new, improved evaluation of the chromatic number. Thus, whenever a new solution is found, any color greater than the best solution is removed from the possible color choices of the other nodes. This best solution is used as the cut-off criterium. In any branch if the numbers of colors used is greater than or equal to the best solution, then EXOC does not proceed along that path any more and cuts-off. The difference between the "depth-first with one child" and a "depth-first search" is that in a "depth-first search" all the branches of the tree are created, all these branches are stored in memory, and each branch is followed until a leaf node of the tree is reached. But in the "depth-first with one child" whenever a new branch of the tree is created, only if its solution is better than the previous solution is the branch kept in memory and the old branch is deleted from memory. Also in 


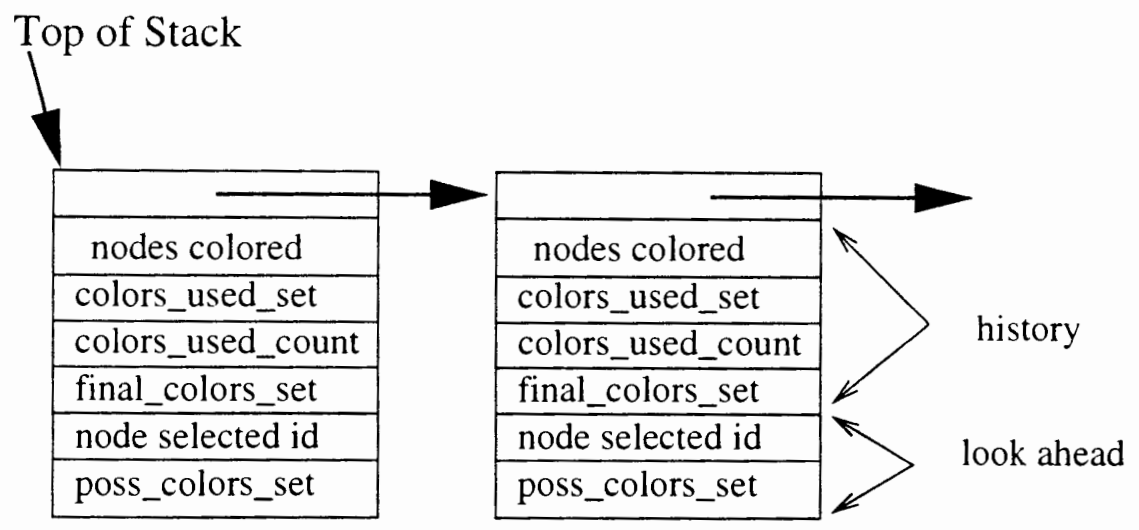

Figure 3.8: Data Structure for a Stack Node used in the Exact Graph Coloring Program

the "depth-first with one child" strategy, because there is a cut-off, EXOC does not go down to the leaf nodes of each branch.

\subsubsection{The Exact Graph Coloring Program Implementation Details}

EXOC was written in ANSI C on a SUN SPARC workstation. Two versions were programmed, one a stand-alone version and one which could be put into the Decomposition programs. Looking at the algorithm it could be seen that a "depth-first search with one child" strategy was needed, because it would provide the ability to cutoff and backtrack. The same would not have been possible with a breadth first search.

There were two possible approaches to implement the "depth-first search with one child" strategy, namely an approach using recursion, or an iterative approach using STACKS. A version using STACKS was chosen here. A STACK is a Last_in_First_out data structure, meaning the last information stored, is the first information which will be retrieved from the STACK.

One STACK is used in the program, the STACK is defined here as a singly linked list structure. Fig 3.8 shows the data structure used to implement the stack. The information held by each stack node can be divided into two parts. The history holds the information about what has happened before this node, 
and the Look Ahead holds the information about the next node. The history holds four pieces of information: nodes_colored is the number of nodes colored before this stage, colors_used_set is the different colors used before this node, colors_used_count is the number of colors used until this node, and final_colors_set is the set of nodes and the colors assigned to the nodes until this node. The Look ahead holds two pieces of information: node_selected which is the node selected for the next node in the tree, and poss_colors_set which is the set of colors that the node selected can be colored with.

There are three main operations done on the STACK which are a PUSH, POP and PEEK. PUSH puts a new node on top of the STACK, and returns the new top of the stack. POP removes the topmost node, frees the memory for that node and returns the new top of the STACK. PEEK looks at the top of the stack, for some information about the topmost node.

In EXOC there are two possible ways to choose the node order, nodes can be chosen in a simple binary order and nodes can be chosen after sorting them in descending order according to the number of neighbors.

Theorem 3.3 The number of colors generated for an incompatibility graph by EXOC will always be equal to the chromatic number of the graph.

Proof 3.3 For an incompatibility graph with $\mathbf{n}$ nodes, each node has at most $\mathbf{n c}$ possible colors that it can be colored where $\mathbf{n c}=\mathbf{n}$. Hence if a depth first search is executed in which a different node is colored at different levels of the tree with all colors in nc which are possible for that node (possible color for a node means none of its neighbors can have the same color) then different solutions will be obtained at the leaves of the tree. The least cost of these solutions will be the chromatic number of this incompatibility graph. Many best colorings of the graph may be arrived at by this search. In the "depth-first with one child" search, for each node all the possible colors in nc for that node are not being checked but only a subset of colors in nc are being checked, but this subset of colors is initially equal to $\mathbf{n}$, and when a solution new_sol is found, then only the number of colors in nc 
which is less than or equal to new_sol is checked. Hence the "depth-first with one child" search will always arrive at one of the leaves of the tree which is the chromatic number of the graph, but will not find all possible minimum colorings for the graph. Hence this proves the Theorem 3.3, that the solution generated by EXOC is always the exact solution.

Since $E X O C$ cuts-off, whenever it finds in a branch of the tree that the number of colors used is equal to or greater than the best solution, the maximum memory of stack nodes used at any one time will be equal to the number of nodes in the incompatibility graph. The speed will depend on when the exact solution is found, in the worst case the exact solution will be found in the leaf node of the last path of the tree traversed, but this will rarely happen, if ever. In the best case the exact solution will be found in the leaf node of the first path of the tree traversed. In the program, to increase the efficiency, the step of cutting-off before calculating poss_colors_set for a node has been applied. From the candidates to color the selected node $S N$, one can select the colors which are different from colors used for nodes that have already been colored, if an edge exists between the $S N$ and an already colored node. In the moment of finding solution in a node it is known that the minimal solution needs at most as many colors as in colors_used_set. Thus once a solution is found, from all poss_colors_set of all nodes any color which is > colors_used_count can be removed, since we are not interested in all possible colorings, but only in the colorings with the accuracy to an isomorphism (i.e the minimum chromatic number colorings). Let us note that two types of nodes are dealt with here: nodes of the graph, and nodes of the search tree.

\subsubsection{An Example showing how EXOC colors a graph}

Figure 3.9 is an example which shows how EXOC colors a graph. In this example the incompatibility graph being colored is a non cyclic graph, but the algorithm for $E X O C$ does not consider if the graph is cyclic or not, it uses the 


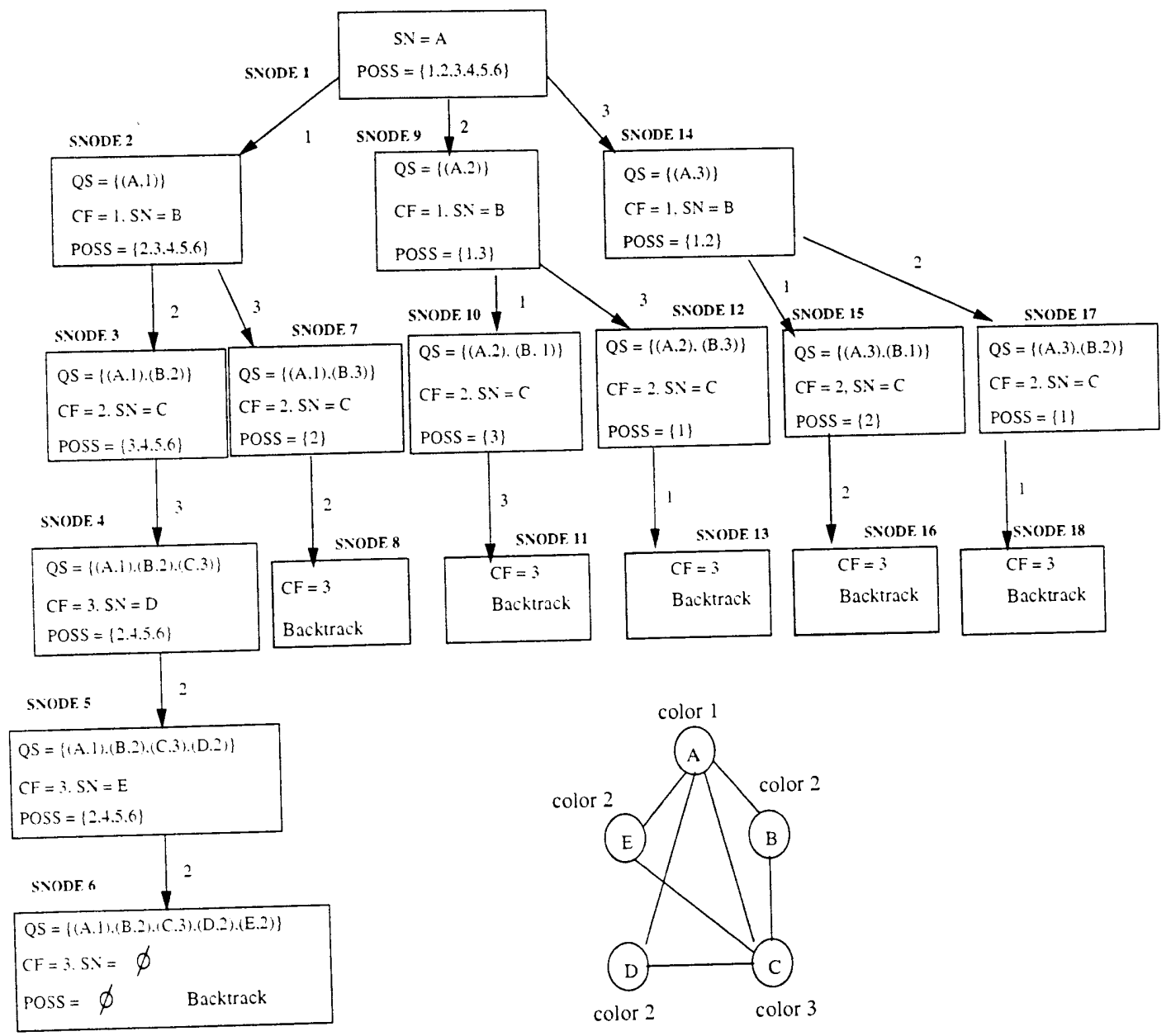

Figure 3.9: Tree Search for the Exact Graph Coloring Algorithm 
same method to color a graph independent of whether the graph is cyclic or not. Since EXOC deals with stack nodes and with nodes of the graph, in each step given below $S N O D E$ refers to a node of the STACK and GNODE refers to a node of the incompatibility graph.

The search process is executed as follows.

1. Select GNODE "A" and find the possible colors for it and push the $S N O D E$ labeled SNODE 1 in Figure 3.9 on to the stack.

Selected color for GNODE "A" is 1 .

2. Now select a new GNODE which is node "B". GNODE "B" can be given the possible colors $2,3,4,5,6$. Store this information on $S N O D E$ labeled SNODE 2 in Figure 3.9.

Selected color is 2 .

3. Now GNODE "C" becomes the selected node and it can obtain one of colors $\{3,4,5,6\}$.

Selected color is 3 .

4. In this way, the first branch of the tree from Figure 3.9 is created. After finding the first solution:

color $1=\{\mathrm{A}\}$,

color $2=\{\mathrm{B}, \mathrm{D}, \mathrm{E}\}$,

color $3=\{\mathrm{C}\}$.

we know that three colors are enough, and these colors are 1,2 and 3 .

5. All non-used colors are then removed from sets $P O S S$ of all previous nodes. Now backtrack, and at each stage check the POSS colors of the node. If there is a POSS color then go along the new path, else ignore and continue backtracking. 
6. On reaching $S N O D E 2$ there is a $P O S S$ color $=3$.

Select this color for GNODE B, and go to SNODE 7 .

7. IN $S N O D E 7, G N O D E$ "C" is the selected node, and it has only one possible color, which is color 2. Select color 2, for GNODE "C" thus reaching $S N O D E 8$.

8. In $S N O D E 8, C F=3$, which is the same as the best solution, which indicates that any solution along this path will at most result in a solution equal to the best solution, but not better. So cutoff and backtrack.

9. Ultimately on reaching $S N O D E 1$, select color 2 for GNODE "A" and go along that path. On reaching $S N O D E 11$ the $C F$ is 3 , so do not proceed any more along this path, and cut off here and backtrack.

10. Figure 3.9 shows all the paths of the tree that are traversed. The SNODEs are labeled in the order in which they are visited.

11. If at any SNODE a solution is obtained that is better than the one obtained at SNODE 6, store the new solution and discard the old solution. Continue till all the possible paths of the tree have been traversed. The solution saved is the minimum coloring of the graph.

\subsubsection{Algorithm for EXOC}

NODES

POSSIBLE_COLORS_SET

COLORS_USED_SET

COLORS_USED_COUNT

FINAL_COLORS

COLORS_USED_MIN

NODE_COUNT is the set of nodes.

is the set of colors possible for a node.

is the set of colors used.

is a count of the number of colors used.

is a set containing ( $N O D E, C O L O R$ ) for

every node.

is the minimum number of colors.

is the number of nodes in the graph. 
$S N$ is the selected node.

Step 1. Creating the Initial State:
a) $N O D E_{1}:=\operatorname{NODES}[0], \quad \quad /^{*}$ take first node from $N O D E S * /$.
b) Find POSSIBLE_COLORS for the node.
c) Remove $N O D E_{1}$ from NODES.
d) COLORS_USED_MIN $=$ NODE_COUNT/* Initial set up */

\section{Step 2. Depth First Search}

a) $S N:=$ first element from $N O D E S$.

b) Choose minimum COLOR from POSSIBLE_COLORS for the node.

c) Remove $S N$ from $N O D E S$

d) if (COLOR $\left.\notin C O L O R S_{-} U S E D_{-} S E T\right)$ then COLORS_USED_COUNT++.

Add new COLOR to COLORS_USED_SET.

Add ( NODE,COLOR) to FINAL_COLORS.

e) else $\quad / *$ No Change in cost */

Add (NODE, COLOR) to FINAL_COLORS.

f) if ( COLORS_USED_COUNT $\leq$ COLORS_USED_MIN ) then

i) if $\left.\left(R E M A I N I N G \_N O D E S\right)==\emptyset\right)$

$/{ }^{*}$ Found better solution */

Store new best solution.

ii) else $\quad / *$ Still a possibility of better solution */

(continue).

g) else $\left.\quad\right|^{*}$ No chance for better solution along this path */ (cut-off, backtrack). 


\subsubsection{Pseudo code for $E X O C$}

Explanation of Variables used in the Pseudo Code of EXOC

Variable Name

Description

Variable Name

Description

Variable Name

Description

Variable Name

Description

Variable Name

Description

Variable Name

Description

:node

:Data Structure of type shown in Figure 3.8, it is a pointer to a single node in the STACK link list.

:node_order

:Pointer to an array of integers, holds the order in which nodes are selected.

:flag

:flag = NO_BEST_SOL means no best sol has

been found

:flag = BEST_SOL means best sol has been found.

:best_sol

:Integer containing the number of colors in the best solution.

:colors_used_count

:Integer count containing the number of colors used in a stack node of the tree.

:final_colors

Description 
nodes.

Explanation of Functions called by $E X O C$

Function

Synopsis

Returns

Function

Synopsis

Returns

Function

Synopsis

Returns

Function

Synopsis

Returns

Function

Synopsis

Returns

Function

Synopsis :sort_nodes.

:sorts nodes according to the number of neighbors.

:array of type integer, containing the sorted nodes.

:find_possible_choices

:Finds the possible colors for a node of the graph.

:pointer to array of type integer, containing the possible colors for the node.

:push_stack

:Pushes a stack node on to the stack.

:Pointer to new top of the stack.

:possible_choices

:Checks if there are any possible color choices for the graph node.

:TRUE if there is a possible color.

:FALSE if there is no possible color.

:stack_peek

:Copies history (shown in Figure 3.8)

onto a new data structure of type stack node.

:Pointer to the new stack node.

:exchange_colors

:Copies new colors assigned to nodes to final_colors 
and removes the old color information.

Returns

:New array of pointers of nodes and colors.

Function

:stack_pop

Synopsis

:Removes the top most node from the stack and frees the memory used for this node.

Returns :Pointer to the new top of the stack.

/* Initialize stack to NULL */

stack_top := NULL;

${ }^{*}$ Decide a node order */

node_order := sort_nodes();

$/{ }^{*}$ Select the first node */

node $:=$ node_order $[0]$;

$/^{*}$ Find the possible colors that it can be colored */

node $\rightarrow$ possible_choices $:=$ find_possible_choices () ;

$/^{*}$ Push node onto stack */

stack_top := push_stack(node);

$/$ Now we are ready to do the depth first search */

while $($ stack_top $\neq$ NULL $)\{\quad / *$ Peek at top of stack* $/$

while ( possible_choices ()$\neq 0)\{$

$/^{*}$ Peek at top of stack and copy history on to new node */

node := stack_peek();

${ }^{*}$ Find possible colors for the node selected */

node $\rightarrow$ possible_colors $:=$ find_possible_colors();

if ( flag $==$ NO_BEST_SOL $)\left\{/^{*}\right.$ We don't have a best sol yet */

if $\left(\right.$ nodes_colored $==$ node_count) $\left\{/^{*}\right.$ We found a best sol $* /$

$1^{*}$ Get the best solution */

best_sol := colors_used_count; 


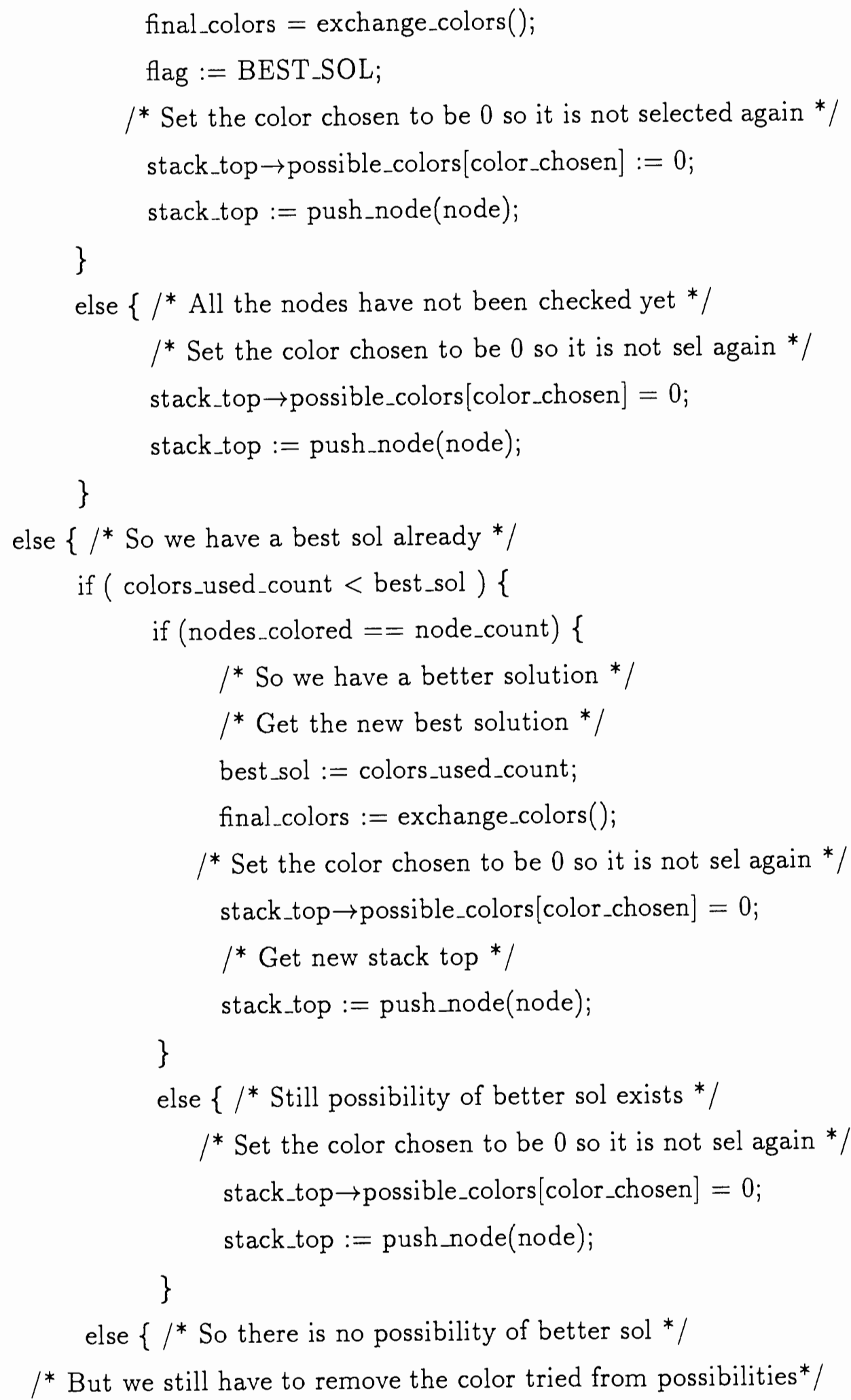


stack_top $\rightarrow$ possible_colors[color_chosen] $:=0$

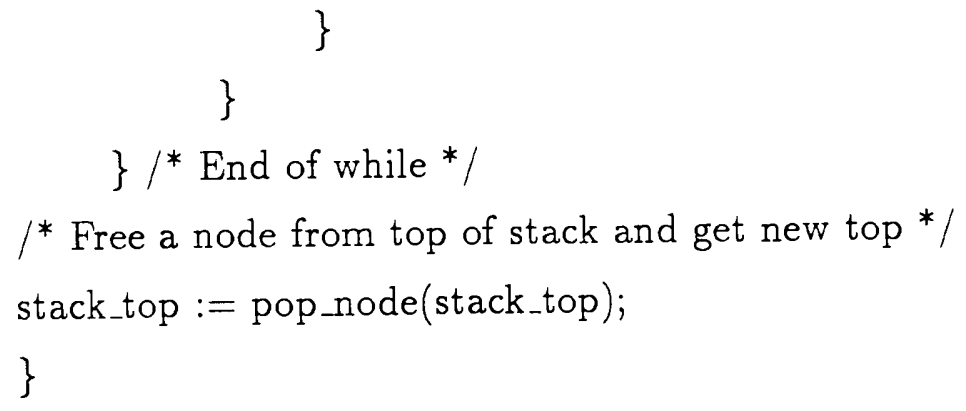

\subsubsection{Future Possible Improvements in EXOC}

1. Theorem 3.4 If two nodes having an edge between them are chosen in the incompatibility graph and given different colors, then there is no need to branch for these two nodes and the solution will be exact.

Proof 3.4 If in an incompatibility graph two nodes having an edge between them are chosen, then they have to be given different colors, and this is not going to change the number of colors in the graph because in all possible colorings of the graph these two nodes have to have different colors.

In the original Algorithm presented by Dr.Perkowski, the first stage started off with 2 nodes already being colored, and for the first node in the tree the selected node is the third node in the graph. But it was found that if these two nodes did not have an edge between them then if a bad color choice is made for these first two nodes then the algorithm will not generate the best solution. As no branching is done for the possible colors of these first two nodes the solution will not be exact if a bad color choice is made for the first two nodes. But as was proved above in Proof 3.4, if the two nodes chosen initially have an edge between them then they can be given different colors and it is not necessary to branch down the tree for any of these initial nodes, hence this is a possible future improvement in the EXOC program. In the original Algorithm presented by Dr.Perkowski, nodes were selected in 
a sequential order, but in $E X O C$, nodes are sorted according to the number of neighbors, and then chosen one by one, instead of being chosen in a sequential order.

2. EXOC can be modified to have a smarter method of choosing the nodes. Possibly the node density or other local graph node-related parameters can be used to evaluate the order, or perhaps the cycle measures or other local graph cycle-related parameters can be used to evaluate the order. When $E X O C$ is used in decomposition, depending on the type of decomposition that is being sought, the upper bound of the number of colors can be passed to EXOC. Therefore EXOC knows what the upper bound of the best solution is, before starting and EXOC will only generate a solution less expensive than the upper bound of the chromatic number if one exists. This would be a big speed up because now EXOC has the upper bound of the minimum solution even before the first pass. If a Curtis binary Decomposition is being sought, then the upper bound on the number of colors is always known. For example if there are 32 nodes in the graph, then it is known that the Bound Set had 5 variables. Now if there are 5 variables in the Bound Set then for a Curtis Decomposition to exist the number of output wires from the $\mathrm{G}$ block must be four or less than four. Now to get an output of four wires the number of colors in the graph of 32 nodes must be no more than 16 for a Curtis Decomposition to exist. Hence for this graph of 32 nodes, number 16 is the upper bound of the number of colors which can be passed to EXOC thus speeding up EXOC.

3. This algorithm can be modified to make it a Multi-Coloring. In Chapter 6 a new heuristic approach to a Multi-Coloring is presented. If EXOC was made into a Multi-Coloring, then it would be a Multi-Coloring with the least possible number of colors. Multi-Coloring will be explained in more detail in Chapter 6. 


\subsection{A New idea for an Exact Graph Coloring Program combining both $D O M$ and $E X O C$}

As was shown earlier in this Chapter, DOM generates the minimum number of colors whenever the resulting incompatibility graph after the check for dominations is a complete graph. Hence it was decided to combine DOM and EXOC together to have a new improved exact graph coloring algorithm. This new algorithm has been given the name DOMEXOC. The advantage of DOMEXOC over $E X O C$ is that it will only call the depth first with one child search of EXOC if the check for dominations did not find a complete graph, thus it will be much faster than EXOC. Also if the check for dominations finds dominations in the incompatibility graph it will remove these dominated nodes from the graph, and hence a depth first search will now be done on a new reduced graph. In order to properly present the algorithm for $D O M E X O C$ first an example is presented which shows how DOMEXOC colors a graph, and then the algorithm for DOMEXOC is presented.

\subsubsection{Example showing how DOMEXOC colors a graph with cycles}

1. Figure $3.10(a)$ shows the incompatibility graph of a function $F$. On checking for dominations in this graph it is found that Node 4 dominates node 6 and pseudo dominates Node 2. Hence Node 6 and Node 2 are removed from the graph. The function which checks for dominations now returns FALSE, cause it cannot find any more dominations in the graph, and the graph is not a complete graph.

2. The new resulting graph is shown in Figure 3.10(b). Now the "depth first with one child" strategy is applied to color this graph. The "depth first with one child" finds the exact minimum number of colors for the graph, resulting in the new graph shown in Figure 3.10(c). 

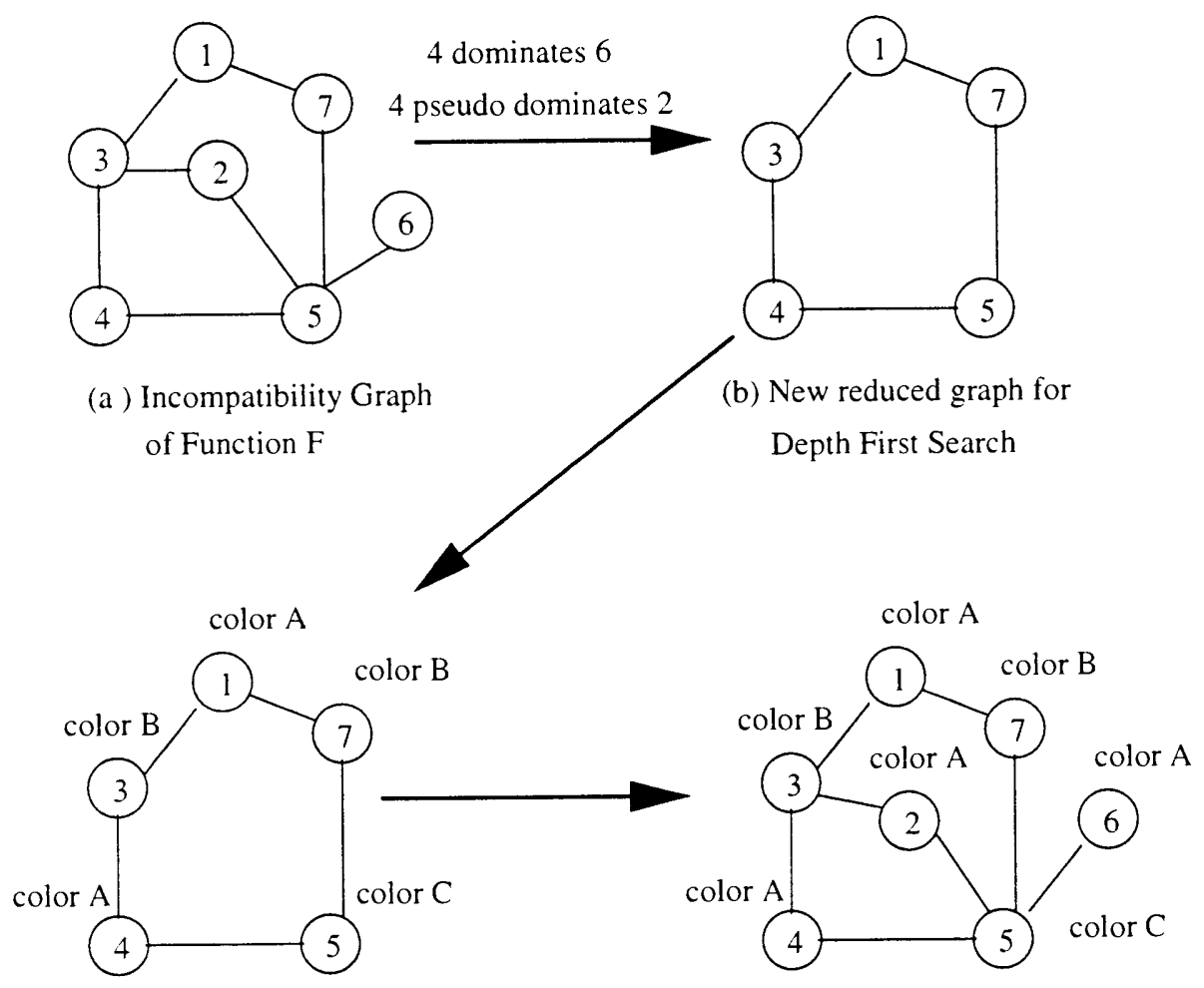

(c) Colored Graph after depth

(d) Final Colored Graph first with one child search

Figure 3.10: An Example showing how DOMEXOC colors a cyclic graph 
3. Now the dominated nodes 2 and 6 are colored with the same color that was assigned to the node 4 which is the node that dominates them. The final color assignment is:

Color $A:\{1,2,4,6\}$,

Color B : $\{3,5\}$,

Color $\mathrm{C}:\{5\}$.

4. As can be seen from the above example the "depth first with one child" has to color a graph with five nodes instead of the original graph with seven nodes.

\subsubsection{Algorithm for DOMEXOC}

The Algorithm for DOMEXOC uses the function check_dominations used by $D O M$, and the function used by EXOC to do the depth first search. Since these algorithms were explained in detail in earlier sections, they will not be repeated here. The Algorithm explained here for DOMEXOC is the top level Algorithm.

NODES : is the number of nodes in the incompatibility graph.

$C Y C_{-} N O D E S:$ is the nodes remaining after function check_dominations has removed some dominated nodes from the incompatibility graph.

Step 1. Call function check_dominations.

if $($ check_dominations $==$ TRUE $) / *$ Is Graph Complete */ go to Step 4

else

go to Step 2.

Step 2. Since check_dominations has already removed the dominated nodes, call the "depth first with one child" search with the new graph. The number of nodes in this new graph is equal to $C Y C_{-} N O D E S$. The "depth first with 
one child" search will return the colored graph.

Go to Step 3.

Step 3. if ( CYC_NODES $==$ NODES $)$

$1^{*}$ This means there were no dominations found

Hence the graph is already colored. */

exit $(0) ; / *$ Exit from program */

else /* This means some dominations were found in the graph */

${ }^{*}$ Color the dominated nodes the same color as the nodes which dominated them */

color_graph();

exit $(0) ;{ }^{*}$ Exit from program */

Step 4. If we arrived here it means that the function check_dominations found a complete graph. So just color the graph, giving the dominated nodes the same color as the nodes which dominate them.

color_graph()

exit $(0) ; / *$ Exit from program */

Since DOMEXOC combines the features of both DOM and EXOC it should be much faster than EXOC and will only use the "depth first with one child" when check_dominations returns FALSE. DOMEXOC has not been programmed yet and hence there are no results for this method. This idea has been presented here as it was felt that this could be something which could be programmed by someone in the Functional Decomposition group in the future by using both the existing programs $D O M$ and $E X O C$.

\subsection{Summary and Conclusions of Chapter 3}

After implementing these two graph coloring programs, DOM and EXOC which were presented in this Chapter, we needed to compare these two programs. In the next Chapter DOM and EXOC are compared on randomly created 
graphs. In Chapter 5 a comparison is made between the $D O M$, the greedy Clique Partitioning, and the EXOC Algorithms in Decomposition applications. 


\section{CHAPTER 4}

\section{A COMPARISON OF THE DOM AND EXOC GRAPH COLORING PROGRAMS ON RANDOM GRAPHS}

In this Chapter an evaluation is done to see how the heuristic Dominance Graph Coloring (DOM) and the Exact Graph Coloring(EXOC) perform on randomly generated graphs. In order to do the evaluation a random graph generator program was written. This random number generator is called $R A N G^{1}$. RANG accepts as its arguments the number of nodes and the percent of edges, and generates a graph. The system time is used as the seed for $R A N G$, thus ensuring that the graphs generated are totally random. In this Chapter first the DOM program was run on randomly generated graphs, and an evaluation of how well $D O M$ performs in terms of time of execution was done. Then both DOM and $E X O C$ were run on the same randomly generated graphs and their results were compared. These evaluations were done to see how DOM and EXOC perform with respect to increasing numbers of nodes with varying percent of edges, and to see how close to exact are the solutions generated by DOM on the randomly generated graphs.

\subsection{Results of running $D O M$ on Randomly generated Graphs}

The results of running $D O M$ on random graphs as a stand alone version are shown in Table 4.1. In the table " $\mathrm{T}$ " represents the user time and " $\mathrm{C}$ " represents the number of colors. These graphs have been generated with nodes from 100 to 900 with $10 \%$ to $90 \%$ of edges. These graphs were generated by the random graph generator. It was found that due to the random nature of the graphs,

\footnotetext{
${ }^{1}$ RANG can be obtained from the directory/stash/polo at Portland State University
} 


\begin{tabular}{|c|c|ccccccccc|}
\hline \multicolumn{10}{|c|}{ Nodes vs Edges } \\
\hline Edge\% & & \multicolumn{10}{|c|}{ Nodes } \\
\hline & & 100 & 200 & 300 & 400 & 500 & 600 & 700 & 800 & 900 \\
\hline $10 \%$ & $\mathrm{~T}(\mathrm{~s})$ & 0.17 & 0.61 & 1.1 & 0.98 & 1.45 & 3.69 & 2.88 & 5.2 & 5.77 \\
& $\mathrm{C}$ & 6 & 10 & 13 & 16 & 18 & 21 & 23 & 26 & 28 \\
\hline $20 \%$ & $\mathrm{~T}(\mathrm{~s})$ & 0.2 & 0.41 & 0.71 & 1.25 & 1.98 & 3.99 & 3.96 & 7.31 & 8.97 \\
& $\mathrm{C}$ & 11 & 16 & 21 & 26 & 31 & 36 & 40 & 44 & 49 \\
\hline $30 \%$ & $\mathrm{~T}(\mathrm{~s})$ & 0.2 & 0.53 & 1.04 & 1.55 & 2.63 & 4.51 & 5.25 & 9.52 & 12.78 \\
& $\mathrm{C}$ & 14 & 22 & 30 & 36 & 44 & 50 & 59 & 62 & 69 \\
\hline $40 \%$ & $\mathrm{~T}(\mathrm{~s})$ & 0.23 & 0.52 & 1.14 & 1.78 & 3.03 & 4.51 & 6.6 & 11.9 & 16.49 \\
& $\mathrm{C}$ & 16 & 28 & 37 & 47 & 57 & 64 & 74 & 80 & 91 \\
\hline $50 \%$ & $\mathrm{~T}(\mathrm{~s})$ & 0.23 & 0.64 & 1.27 & 2.07 & 3.76 & 5.51 & 7.81 & 15.14 & 20.93 \\
& $\mathrm{C}$ & 21 & 34 & 48 & 60 & 73 & 83 & 94 & 104 & 115 \\
\hline $60 \%$ & $\mathrm{~T}(\mathrm{~s})$ & 0.30 & 0.82 & 1.32 & 2.40 & 4.76 & 6.51 & 9.8 & 18.41 & 25.38 \\
& $\mathrm{C}$ & 23 & 42 & 57 & 72 & 86 & 105 & 115 & 130 & 142 \\
\hline $70 \%$ & $\mathrm{~T}(\mathrm{~s})$ & 0.28 & 0.75 & 1.53 & 2.86 & 5.04 & 7.67 & 11.35 & 21.25 & 30.92 \\
& $\mathrm{C}$ & 28 & 49 & 69 & 92 & 110 & 122 & 140 & 159 & 176 \\
\hline $80 \%$ & $\mathrm{~T}(\mathrm{~s})$ & 0.28 & 1.03 & 1.83 & 3.26 & 5.39 & 8.32 & 12.59 & 26.35 & 37.95 \\
& $\mathrm{C}$ & 36 & 61 & 88 & 113 & 135 & 156 & 178 & 201 & 223 \\
\hline $90 \%$ & $\mathrm{~T}(\mathrm{~s})$ & 0.31 & 0.93 & 2 & 3.97 & 6.21 & 9.95 & 14.93 & 31.84 & 45.49 \\
& $\mathrm{C}$ & 45 & 78 & 114 & 142 & 176 & 203 & 231 & 259 & 290 \\
\hline
\end{tabular}

Table 4.1: Nodes vs Edge Percent for DOM on randomly generated graphs

dominations are rarely found, which is the basis of DOM. Still DOM is fast, and on small graphs the results can be verified, but on large graphs the results cannot be verified.

To evaluate the efficiency of $D O M$ a graph of Nodes vs time has been plotted which is shown in Figure 4.1 for different percentage of edges. Nodes are shown along the $\mathrm{X}$ axis, and time in seconds is shown along the $\mathrm{Y}$ axis. By statistical 


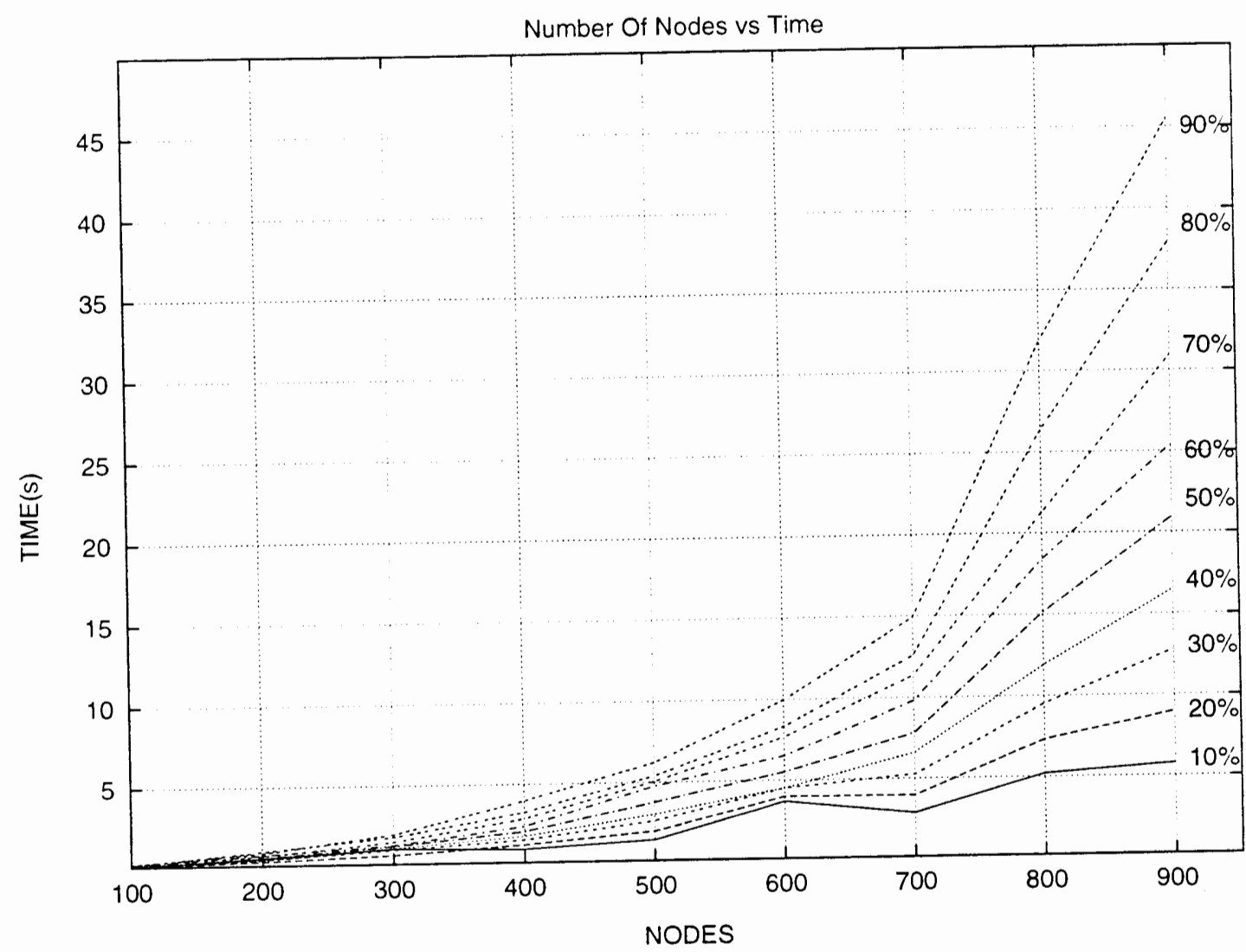

Figure 4.1: Plot of Nodes vs Time for DOM on graphs with increasing number of nodes with different percent of Edges

analysis it was found that $T \propto N^{4.3}$.

\subsection{Comparison of DOM and EXOC on Randomly generated Graphs}

In order to evaluate $D O M$ in terms of the quality of solutions generated by it, a comparison was made by running both $D O M$ and EXOC on the same graphs. The following Tables show the results of the testing.

\subsubsection{Notations used in the Tables}

E.P : Edge Percent

$\mathrm{P} \quad$ : Program

E1 : EXOC with sorted order of nodes 
E2 : EXOC with nodes in sequential order

D $\quad: D O M$

T : Time in Seconds

C : Number of Colors generated

N.B : Number of backtracks required.

1 means best solution was found in the first pass,

2 means best solution was found in the second pass

(number) Number of colors DOM was away from the best solution

\begin{tabular}{|c|c|c|c|c|c|c|c|c|c|c|c|c|c|}
\hline \multicolumn{14}{|c|}{ Edge percent from $10 \%-45 \%$ in steps of $5 \%$} \\
\hline \multirow{3}{*}{ EP } & \multirow{3}{*}{$P$} & \multicolumn{12}{|c|}{ Nodes } \\
\hline & & \multicolumn{3}{|c|}{10} & \multicolumn{3}{|c|}{20} & \multicolumn{3}{|c|}{30} & \multicolumn{3}{|c|}{40} \\
\hline & & $\mathrm{T}(\mathbf{s})$ & $\mathrm{C}$ & T.B & $T(\mathbf{s})$ & $\mathbf{C}$ & T.B & $\mathrm{T}(\mathbf{s})$ & $\mathrm{C}$ & $\overline{T . B}$ & $T(s)$ & $\mathrm{C}$ & T.B \\
\hline \multirow{3}{*}{$10 \%$} & $\overline{E 1}$ & 0 & 2 & 1 & 0 & 3 & 1 & 0 & 3 & 1 & $\overline{0}$ & 4 & 1 \\
\hline & $\mathrm{E} 2$ & 0 & 2 & 1 & 0 & 3 & 1 & 0 & 3 & 2 & 0.1 & 4 & 1 \\
\hline & $\mathrm{D}$ & 0 & 2 & & 0 & 2 & & 0 & 3 & & 0 & 4 & \\
\hline \multirow{3}{*}{$15 \%$} & E1 & 0 & 3 & 1 & 0 & 2 & 2 & 0 & 3 & 1 & 0 & 5 & 1 \\
\hline & E2 & 0 & 3 & 1 & 0 & 2 & 2 & 0 & 3 & 1 & 0 & 5 & 1 \\
\hline & D & 0 & 3 & & 0 & $3(1)$ & & 0 & 3 & & 0 & 5 & \\
\hline \multirow{3}{*}{$20 \%$} & E1 & 0 & 3 & $\overline{1}$ & 0 & 4 & 1 & 0 & $\overline{4}$ & 1 & 0 & 5 & 1 \\
\hline & E2 & 0 & 3 & 1 & 0.3 & 4 & 2 & 2.9 & 4 & 1 & 1.5 & 5 & 2 \\
\hline & $\mathrm{D}$ & 0 & 3 & & 0 & 4 & & 0 & 4 & & 0 & $6(1)$ & \\
\hline \multirow{3}{*}{$25 \%$} & $\overline{E 1}$ & 0 & 3 & 1 & 0 & 4 & 1 & 0 & 5 & 2 & 0.6 & 6 & 1 \\
\hline & $\mathrm{E} 2$ & 0 & 3 & 1 & 0.4 & 4 & 2 & 10 & 5 & 1 & 180 & 6 & 1 \\
\hline & $D$ & 0 & 3 & & 0 & 4 & & 0 & 6(1) & & 0 & $7(1)$ & \\
\hline \multirow{3}{*}{$30 \%$} & E1 & 0 & 3 & 1 & 0 & 4 & 1 & 0.7 & 5 & 1 & 10.5 & 6 & 2 \\
\hline & E2 & 81 & 3 & 1 & 2 & 4 & 2 & 40.7 & 5 & 3 & 15.9 & 6 & 2 \\
\hline & $\mathrm{D}$ & 0 & 3 & & 0 & $5(1)$ & & 0 & 5 & & 0 & $7(2)$ & \\
\hline \multirow{3}{*}{$35 \%$} & E1 & 0.1 & 3 & 1 & 0 & 4 & 2 & 3.5 & 5 & 1 & 39 & 6 & 1 \\
\hline & $\mathrm{E} 2$ & 0.2 & 3 & 1 & 205 & 4 & 2 & 79 & 5 & 3 & 249 & 6 & 3 \\
\hline & $\mathrm{D}$ & 0 & 3 & & 0 & $5(1)$ & & 0 & $6(1)$ & & 0 & $8(2)$ & \\
\hline
\end{tabular}

Table 4.2: Comparison of EXOC and DOM on randomly generated graphs with edge percent from 10 to 35

In Table 4.2 EXOC was run both with nodes in sorted order and with nodes in sequential order. In this Table, nodes were varied from 10 to 40 in steps of 10 , and edge percent was varied from $10 \%$ to $35 \%$ in steps of $5 \%$. The following is a summary of the results obtained in Table 4.2.

1. Total Number of Program Runs $=24$. 
2. The version of $E X O C$ which selected nodes after sorting them according to the number of edges(E1), found the solution in the first pass in 20 cases, and in the second pass in 4 cases. The version of EXOC which selected nodes sequentially, found the solution in the first pass in 13 cases, in the second pass in 8 cases, and in the third pass in 3 cases.

3. DOM found the best solution in 15 cases, was one color away from the best solution in 7 cases and was two colors away from the best solution in 2 cases. The cases when $D O M$ did not find the best solution are shown in bold in Table 4.2 .

\begin{tabular}{|c|c|c|c|c|c|c|c|c|c|c|}
\hline \multicolumn{11}{|c|}{ Edge percent from $40 \%-90 \%$ in steps of $10 \%$} \\
\hline \multirow{3}{*}{$\mathrm{EP}$} & \multirow{3}{*}{$P$} & \multicolumn{9}{|c|}{ Nodes } \\
\hline & & \multicolumn{3}{|c|}{10} & \multicolumn{3}{|c|}{20} & \multicolumn{3}{|c|}{30} \\
\hline & & $\mathrm{T}(\mathrm{s})$ & $\mathrm{C}$ & T.B & $\mathrm{T}(\mathbf{s})$ & $\mathrm{C}$ & T.B & $\mathrm{T}(\mathbf{s})$ & $\mathrm{C}$ & T.B \\
\hline \multirow[t]{2}{*}{$40 \%$} & E1 & 0 & 4 & 1 & 0.1 & 5 & 1 & 0.8 & 6 & 2 \\
\hline & D & 0 & 4 & & 0 & 5 & & 0 & $7(1)$ & \\
\hline \multirow[t]{2}{*}{$50 \%$} & $\overline{E 1}$ & 0 & 4 & 1 & 1.5 & 6 & 1 & 1200 & 8 & 2 \\
\hline & $\mathrm{D}$ & 0 & 4 & & 0 & 6 & & 0 & $\theta(1)$ & \\
\hline \multirow[t]{2}{*}{$60 \%$} & E1 & 0 & 5 & 1 & 6.8 & 7 & 1 & 12000 & 9 & 2 \\
\hline & $\mathrm{D}$ & 0 & 5 & & 0 & 7 & & 0 & $11(2)$ & \\
\hline \multirow[t]{2}{*}{$70 \%$} & E1 & 0 & 5 & 1 & 16.8 & 8 & 2 & 18000 & 10 & 2 \\
\hline & $\mathrm{D}$ & 0 & 5 & & 0 & $\theta(1)$ & & 0 & $12(2)$ & \\
\hline \multirow[t]{2}{*}{$80 \%$} & E1 & 0 & 5 & 1 & - & - & & - & - & \\
\hline & $\mathrm{D}$ & 0 & 5 & & 0 & 10 & & 0 & 16 & \\
\hline \multirow[t]{2}{*}{$90 \%$} & E1 & 0 & 7 & 1 & 3600 & 11 & 1 & - & 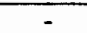 & \\
\hline & D & 0 & 7 & & 0 & 11 & & 0 & 16 & \\
\hline
\end{tabular}

Table 4.3: Comparison of EXOC and DOM on randomly generated graphs with edge percent from 40 to 90

In Table 4.3 DOM and the version of EXOC which selected nodes after sorting according to the number of edges(E1) were run, on graphs with nodes from 10 to 30 in steps of 10 and edge percent from $40 \%$ to $90 \%$ in steps of $10 \%$. The following is a summary of the results of Table 4.3 .

1. Total Number of Program Runs $=18$.

2. The version of $E X O C$ which selected nodes after sorting according to the number of edges, found the solution in the first pass in 10 cases, in the second pass in 5 cases, and EXOC was terminated in 3 cases, as EXOC took too long( time greater than $18000 \mathrm{sec}$ ). 
3. DOM found the best solution in 10 cases, was one color away from the best solution in 3 cases, and was two colors away from the best solution in 2 cases.

\subsection{Summary and Conclusions of Chapter 4}

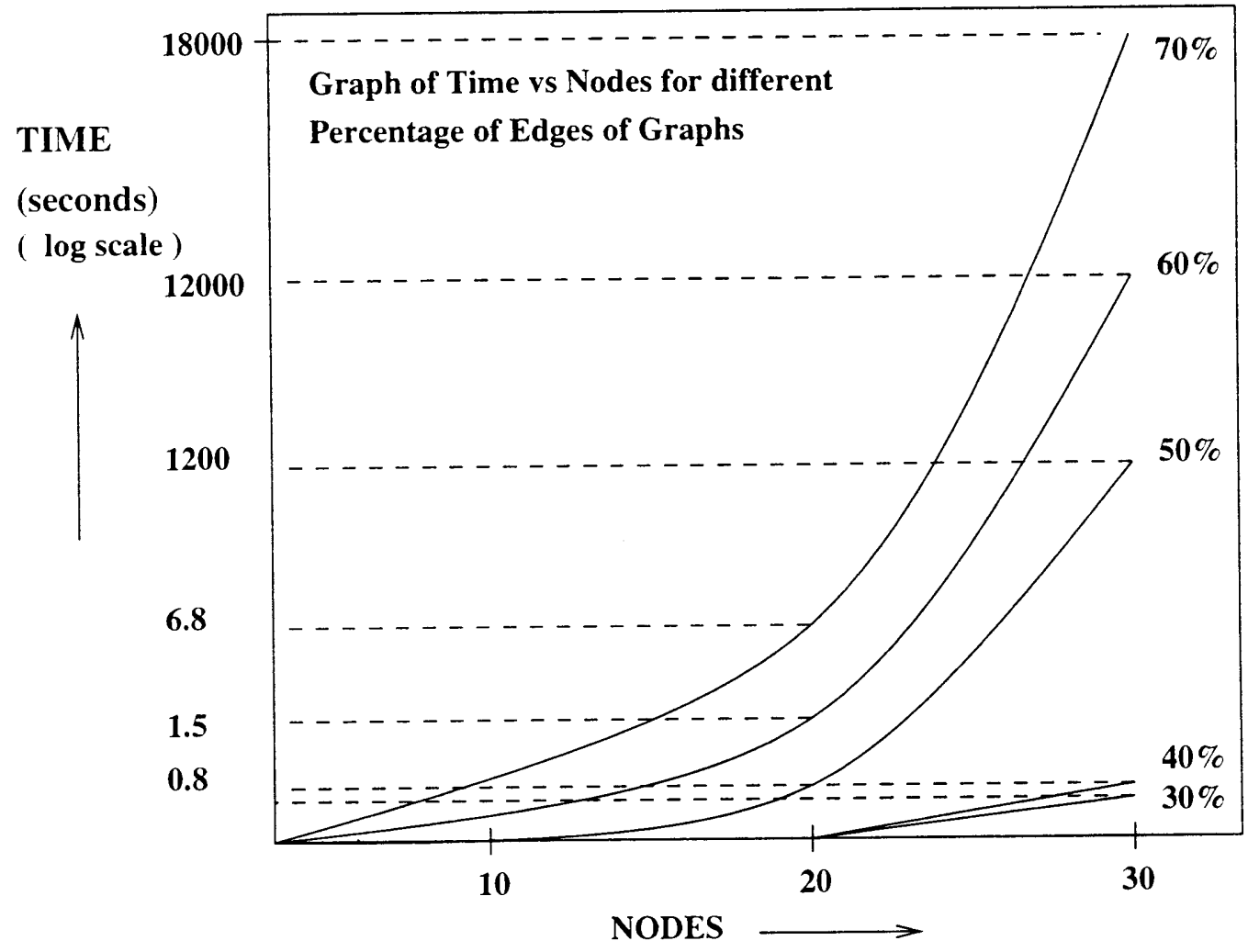

( log scale )

Figure 4.2: Graph of Nodes vs Time showing how EXOC performs on randomly generated graphs with edge percent from $30 \%$ to $70 \%$

In order to evaluate the results obtained in Table 4.2 and Table 4.3, a graph of nodes vs time for varying percent of edges was plotted for EXOC. Figure 4.2 shows the plot. As can be seen from the plot as the number of nodes increases, for graphs having percentage of edges up-to $40 \%$ the time taken by EXOC is a maximum of $0.8 \mathrm{sec}$ which is very fast. But once the percent of edges increases 


\begin{tabular}{|c|c|c|c|c|c|}
\hline \multicolumn{6}{|c|}{ Number of Program Runs $=39$} \\
\hline \multicolumn{2}{|c|}{ Times Sol Exact } & \multicolumn{3}{|c|}{ Times Sol 1 color away } & \multicolumn{2}{|c|}{ Times Sol 2 colors away } \\
\hline Number & Percent & Number & Percent & Number & Percent \\
\hline 25 & $64 \%$ & 10 & $25.6 \%$ & 4 & $10 \%$ \\
\hline
\end{tabular}

Figure 4.3: Summary of Results showing how close to exact are the solutions generated by $D O M$ on randomly generated graphs

to $50 \%$ the time EXOC takes to color a graph with 30 nodes and $50 \%$ of edges is 1200 seconds, and for a graph with 30 nodes and an edge percent of 60 , the time increases by a 1000 times and becomes 12000 secs.

Observation 4.1 When the chromatic number of a graph is high EXOC is going to be slow.

To support this observation note that when there is a high percent of edges in a graph, the number of colors in the best solution is going to be high that is the chromatic number is going to be high. Hence each node is going to have a high number of possible colors, with the number of possible colors being highest for the node which is colored first. Now if there is a high number of possible colors, and if the best solution itself is high each possible color which is smaller or equal to the best solution has to be tried, and this is can be a very large search space. These results indicate that for graphs with 30 or more nodes and more than $50 \%$ of edges, it is not so important to have a good node order, or even to have the chromatic number passed into $E X O C$, but only if the graph has a low chromatic number will the speed of $E X O C$ be faster 4.1. This suggests that EXOC should only be used for applications in which the size of the graphs are less than 40 nodes, and in the graphs having more than 30 nodes, the percentage of edges should be less than $50 \%$.

Table 4.3 shows how close to exact are the solutions generated by DOM. As can be seen $D O M$ generates the exact solution in $64 \%$ of cases, is one color away from 
the best solution in $25.6 \%$ of cases, and in $10 \%$ of cases is two colors away from the best solution. Thus this suggests that, if the application does not require an exact solution at all times, and speed is the primary criterium, then DOM will be better than EXOC.

As the next step, we are going to test DOM and EXOC in Functional Decomposition. Since in Functional Decomposition, speed is of great importance to us, we know that if the graphs generated during decomposition fall in the category where they have a high percent of edges, then, even if EXOC generates better results than the heuristic methods, it will not be a practical method, since it will be too slow. This is presented in the next Chapter. In the next Chapter we also want to answer the following questions:

1. Does generating exact results for the graphs at every step of the Decomposition, improve the quality of the decomposition achieved?

2. Are the results generated by $D O M$ for the graphs generated during the steps in Functional Decomposition for real life functions going to be better than the results generated for random graphs? 


\section{CHAPTER 5}

\section{AN EVALUATION OF THE PROBLEM OF COLUMN MULTIPLICITY IN DECOMPOSITION OF FUNCTIONS}

In this Chapter we will evaluate the importance of an Exact Graph Coloring in Curtis Decompositions. Our aim is to investigate if an Exact Graph Coloring is required in Functional Decomposition and if it leads to better results on the graphs that are created from practical function benchmarks. We used the Decomposer Multi-Valued General Universal DecomposerMVGUD written at Portland State University for the testing purpose. We instantiated three algorithms into MVGUD, a Greedy Clique Partitioning, the Dominance Graph Coloring $(D O M)$ and the Exact Graph Coloring $(E X O C)$. The decomposer was run with different numbers of variables in the Bound Set on two kinds of benchmarks: $M C N C$ benchmarks for circuits, and Machine Learning Benchmarks(from the Wright Labs Database) for data from Machine Learning, Pattern Recognition and Knowledge Discovery in Data Bases.

In the previous Chapter a comparison of DOM and EXOC was done on randomly generated graphs, for varying number of nodes and varying percentage of edges. Thus, in the previous Chapter, conclusions were reached about how well DOM and $E X O C$ will perform on the different kinds of graphs. So here too tests were done to characterize the kind of graphs that are generated in decomposition with regard to the number of nodes in the graph and the percentage of edges in the graph in order to see if the same conclusions hold for the graphs generated during Functional Decomposition. In this Chapter first some notions and definitions are presented, then a brief introduction to $M V G U D$ is presented, in which the strategy used by $M V G U D$ to perform decompositions is explained and then the 


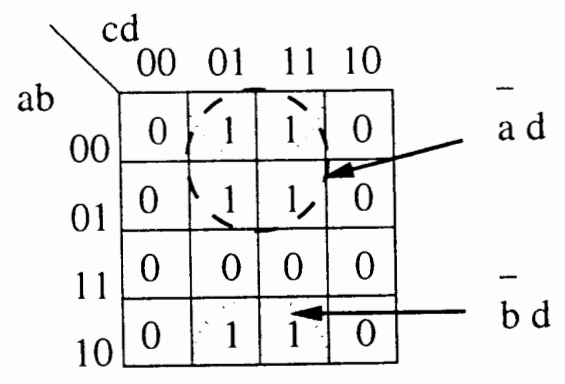

Figure 5.1: The Karnaugh map of a function showing a Vacuous Variable

evaluation of the Column Multiplicity problem in Functional Decomposition is presented.

\subsection{Some Notions and Definitions}

\subsubsection{A Vacuous Variable}

Definition 5.1 A vacuous variable in a Function $F$ is an input variable which is not needed to realize Function $F$. Removing a vacuous variable will not change the function.

Figure 5.1 shows a Karnaugh map of a function having four binary inputs and one binary output. On solving the Karnaugh map it can be seen that input variable " $c$ " does not appear in the solution, hence input variable " $c$ " is not needed to realize the function, and it can be removed.

\subsubsection{Clique Partitioning and Clique Covering}

Since $M V G U D$ has a greedy Clique Partitioning incorporated in it, here a Clique Partitioning, and the difference between a Clique Partitioning and a Clique Covering is presented. 


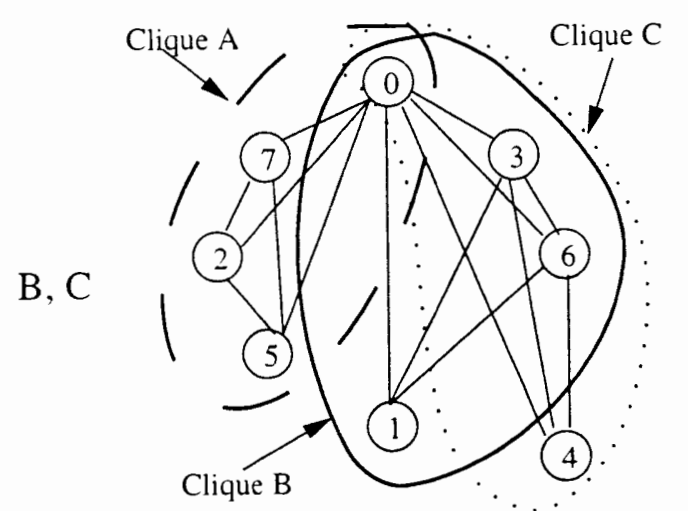

(a) Compatibility Graph with

Clique Covering.

Maximum Cliques:

Clique $\mathrm{A}\{0,2,5,7\}$

Clique $B\{0,1,3,6\}$

Clique $C\{0,3,4,6\}$

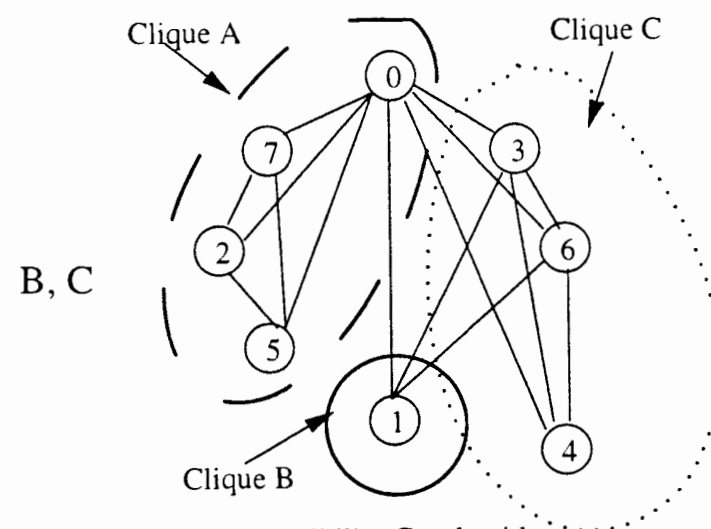

(b) Compatibility Graph with

Clique Partitioning.

Cliques:

Clique $\mathrm{A}\{0,2,5,7\}$

Clique B $\{1\}$

Clique $C\{3,4,6\}$

Figure 5.2: The Difference between Clique Covering and Clique Partitioning

\subsubsection{Clique Partitioning of a Compatibility Graph}

Definition 5.2 Clique Partitioning of a Graph $G=(V, E)$ is a partitioning of the vertex sets of $G$ into independent sets (color classes). These independent sets are called cliques.

Clique Partitioning is done on an Compatibility Graph, in Clique Partitioning there is no overlap between the Cliques. Figure 5.2(b) shows a Clique Partitioning of a graph into three cliques, as can be seen there is no overlap between the cliques.

\subsubsection{Clique Covering of a Compatibility Graph}

Definition 5.3 Clique Covering of a Graph $G=(V, E)$ is to find a set of cliques of minimum cardinality whose union covers all the vertices of the graph.

Figure 5.2(a) shows a Clique Covering of a graph. As can be seen here, there are still three cliques, but now there is an overlap between the cliques. 


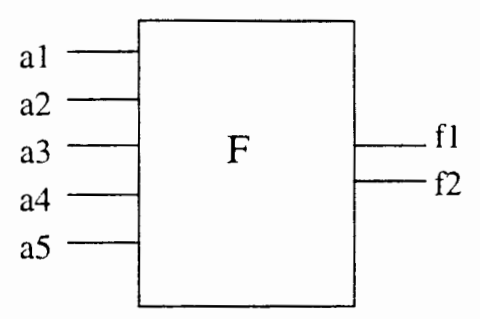

$$
\mathrm{DFC}=2^{5} * 2=64
$$

(a ) DFC Calculation in a Binary Function

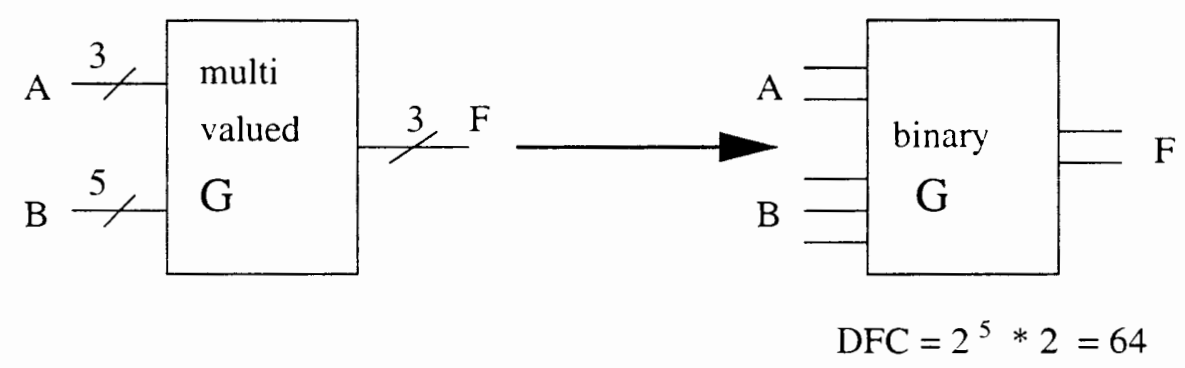

( b ) DFC Calculation in a Multi-Valued Function

Figure 5.3: Calculation of DFC in Binary and Multi-Valued Functions

\subsubsection{Decomposed Function Cardinality}

Definition 5.4 Decomposed Function Cardinality(DFC) for a binary function is a measure of a function complexity and is defined as $2^{N} * M$, where $N$ is the number of inputs and $M$ is the number of outputs of the binary function.

DFC is defined as the cost of the minimum Decomposition. DFC is a good evaluation of the Decomposition quality for Machine Learning Applications but it does not take into account the complexity of the decomposed blocks. Figure 5.3(a) shows how DFC is calculated for a Binary Function.

For multi-valued functions, DFC has not been defined as a standard yet. In order to calculate the $D F C$ of the resulting function, $M V G U D$ translates the multi valued inputs and outputs of each block in the final Decomposition into their binary equivalent ( $\log _{2}$ multi_value) and then calculates the equivalent 
binary $D F C$. In Figure 5.3(b) input variable A which is 3-valued, is converted to 2 bits, and input $B$ which is 5 -valued is converted to 3 bits. The output is converted to 2 bits. Then the formula used to calculate the binary $D F C$ is used resulting in a DFC of 64 .

\subsection{An Introduction to MVGUD}

MVGUD [26] is a multi-valued decomposition program written by Stanislaw Grygiel at Portland State University. The following algorithm explains the strategy used by $M V G U D$ to perform multi-valued decompositions. This algorithm explains one step of the decomposition process.

\subsubsection{Strategy used by $M V G U D$ to perform Decompositions}

Step 1 For an input function $F$ : Generate a set of Disjoint Partitions:

$$
P A R T_{-} S E T=\left\{\left(B_{1}, A_{1}\right),\left(B_{2}, A_{2}\right),\left(B_{i}, A_{i}\right), \cdots\left(B_{n}, A_{n}\right)\right\}
$$

where $n=8 \cdots 16, B_{i}$ is the Bound set and $A_{i}$ is the free set of Partition $i$ of the Function $F$. Size of the Bound Set is an input parameter to the program MVGUD. These partitions are generated using Wan Wei's [6] algorithms for Variable Partitioning.

Step 2 Set initial column multiplicity $\left(\mu_{\min }\right)$ to a high number.

Step 3 Choose a partition $\left(B_{i}, A_{i}\right)$ from $P A R T_{-} S E T$.

Call the clique partitioning/graph coloring program to calculate the column multiplicity $\left(\mu_{B_{i}, A_{i}}\right)$ of chosen Partition $\left\{\left(B_{i}, A_{i}\right)\right\}$.

Step 4 If $\mu_{B_{i}, A_{i}}<\mu_{\min } \quad$ then set

$\mu_{\min }=\mu_{B_{i}, A_{i}}$, go to Step 5 .

If $\mu_{B_{i}, A_{i}}=1$, break out and return 1 because this means that all the Variables in the Bound Set are vacuous. 
Step 5 If all Partitions in PART_SET have not been tried, go to Step 3 else go to Step 6.

Step 6 Now check if the resulting $\mu_{\min }$ is an acceptable Curtis Decomposition. In order to do this find the highest multi-value in the inputs of the Bound set $B_{i}=m$, and let the number of variables in the Bound set $B_{i}=n$. Here $B_{i}$ is the Bound set belonging to the PART_SET $=\left\{\left(B_{i}, A_{i}\right)\right\}$ which resulted in the best $\mu_{\text {min }}$. Now if $n>\log _{m}\left(\mu_{\min }\right)$ then it is an acceptable Curtis decomposition. This decision made by $M V G U D$ to decide if a $\mu$ is an acceptable Curtis decomposition, can be better understood by considering a Binary decomposition. If a function $F$ has $n$ binary inputs, then for a Curtis Decomposition to exist $n>\log _{2} \mu$. Hence the same reasoning is applied to a multi-valued function, only now the base of the log is the highest multivalued input of the Function. If an acceptable decomposition was found then return with the decomposition found. If it is not an acceptable Curtis Decomposition, then increase size of the bound set and go back to Step 1 .

Step 7 If the size of the bound set is one less than number of inputs and still no decomposition is found, go back to Step 1, only now generate-non disjoint partitions.

Since $M V G U D$ is a multi-valued decomposer, it has no encoding stage. Essentially $M V G U D$ looks for the Curtis Binary Decomposition criteria which was explained in Chapter 2 in evaluating if a decomposition is acceptable, but then assigns the output of the " $G$ " function one value which is equal to the $\mu_{\min }$ found. This approach results in one multi-valued output from each " $G$ " block, which can be called a multi-valued Ashenhurst Decomposition. Whether the method used by $M V G U D$ to calculate $D F C$ (explained in Section 5.1.4), is a good evaluation of the cost of the decomposed multi-valued blocks is not known, but since the $D F C$ is used for a comparison between different methods of calculating the Column Multiplicity in Decomposition, within the same decomposer, the method of 
calculation of the DFC does not matter for the purpose of evaluating algorithms for calculating column multiplicity. What matters is that the same method is used for all the algorithms that are compared.

\subsection{A Comparison of the different Strategies of finding the Column Multiplicity in Functional Decomposition}

How the different algorithms for calculating the Column Multiplicity in Functional Decomposition compare on functions from Circuit, and Machine Learning Benchmarks will be presented in this section. Here a comparison is done between $D O M, E X O C$ and the heuristic Clique Partitioning Program which we will call CLIP. The goal of this testing is to see if an Exact Graph Coloring is necessary to calculate the Column Multiplicity in Functional Decomposition, and if the $D F C$ can be improved in case that $M V G U D$ is run with $E X O C$, in comparison to when it is run with $D O M$ or with $C L I P$. The results of the testing have been divided into two parts, first the testing is done on the Circuit benchmarks, and then the testing is done on the Machine Learning Benchmarks. MVGUD was tested with two, four, and five variables in the Bound set.

\subsubsection{Notations Used in the Tables}

The following is an explanation of the Notations used in the Tables in this Chapter.

1. Benchmark : Name of the Benchmark function.

2. in : Number of inputs of the Benchmark.

3. out: Number of outputs of the Benchmark.

4. cubes: Number of cubes in the Benchmark.

5. DFC : Decomposed function cardinality of the decomposed function. 
6. Algorithm: Name of Algorithm used in MVGUD.

7. Nu of Blocks : Number of multi-valued blocks in the decomposed function.

8. NP : Number of passes, or number of times the function to calculate the column multiplicity was called.

9. TC : Total Colors, iterative sum of colors generated for each pass.

10. AC : Average Colors $=\mathrm{TC} / \mathrm{NP}$.

11. $\mathrm{T}(\mathrm{s})$ : User time in seconds.

\subsubsection{A Comparison of the different Strategies of finding the Column Multiplicity on MCNC Benchmarks}

\subsubsection{A Comparison when $M V G U D$ is run with 2 variables in the Bound Set}

Table 5.1 shows a comparison made by running $M V G U D$ with 2 variables in the Bound Set for EXOC, DOM and CLIP. Comparisons were made with respect to the DFC, the number of two-input gates in the final decomposed function, and the time taken by $M V G U D$ to decompose the function. In Table $5.12 \mathrm{i} / \mathrm{p}$ $g$ stands for the equivalent number of two input gates. This is calculated by MVGUD.

As can be seen from Table 5.1,DOM and CLIP both perform well in different cases. DOM provides a smaller DFC in 6 cases and CLIP provides a smaller DFC in 5 cases, while EXOC provides a tie with the best solution in 6 of the cases. Hence EXOC does not provide any real improvement, on the other hand it is much slower than $C L I P$ and $D O M$, which is to be expected. The reason for this kind of results is that since in this experiment there are 2 variables in the Bound Set, in most cases the size of the Incompatibility Graph at different levels of the decomposition is 4 , which is a small graph, and it is known that for small graphs both CLIP and DOM usually generate the best solution as well. Hence 


\begin{tabular}{|c|c|c|c|c|c|c|c|c|c|c|c|}
\hline \multicolumn{12}{|c|}{ 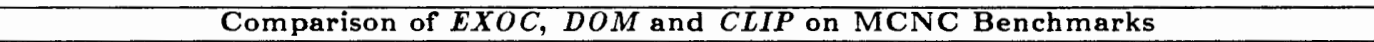 } \\
\hline Benchmark & in & out & cubes & Algorithm & $\overline{\mathrm{DFC}}$ & $\begin{array}{l}\text { nu of } \\
\text { blocks }\end{array}$ & $2 \mathrm{i} / \mathrm{pg}$ & $\mathrm{T}(\mathbf{s})$ & NP & TC & $\overline{\mathrm{AC}}$ \\
\hline \multirow[t]{3}{*}{$5 \times p 1$} & \multirow[t]{3}{*}{7} & \multirow[t]{3}{*}{10} & \multirow[t]{3}{*}{143} & EXOC & 274 & 36 & 69 & 76 & 168 & 613 & 3.6 \\
\hline & & & & $D O M$ & 274 & 36 & 69 & 10.5 & 168 & 611 & 3.63 \\
\hline & & & & $C L I P$ & 314 & 34 & 79 & 10.6 & 163 & 589 & 3.6 \\
\hline \multirow[t]{3}{*}{$9 \mathrm{sym} 1$} & \multirow[t]{3}{*}{9} & \multirow[t]{3}{*}{1} & \multirow[t]{3}{*}{158} & $E X O C$ & 112 & 4 & 28 & 103 & 38 & 146 & 3.8 \\
\hline & & & & $D O M$ & 60 & 5 & 15 & 29.7 & 38 & 146 & 3.8 \\
\hline & & & & $C L I P$ & 112 & 4 & 28 & 31.2 & 34 & 136 & 4 \\
\hline \multirow[t]{3}{*}{$\mathrm{b} 12$} & \multirow[t]{3}{*}{15} & \multirow[t]{3}{*}{9} & \multirow[t]{3}{*}{172} & $\overline{E X O C}$ & 302 & 52 & 76 & 84 & 353 & 940 & 2.66 \\
\hline & & & & $D O M$ & 302 & 52 & 76 & 12.2 & 353 & 940 & 2.66 \\
\hline & & & & $C L I P$ & 302 & 52 & 76 & 10.9 & 351 & 924 & 2.64 \\
\hline \multirow[t]{3}{*}{$\operatorname{con} 1$} & \multirow[t]{3}{*}{7} & \multirow[t]{3}{*}{2} & \multirow[t]{3}{*}{18} & $E X O C$ & 90 & 11 & 23 & 8.3 & 58 & 230 & 3.9 \\
\hline & & & & $D O M$ & 94 & 10 & 24 & 8.0 & 58 & 230 & 3.9 \\
\hline & & & & $C L I P$ & 90 & 11 & 23 & 7.9 & 55 & 204 & 3.71 \\
\hline \multirow[t]{3}{*}{ bw } & \multirow[t]{3}{*}{5} & \multirow[t]{3}{*}{28} & \multirow[t]{3}{*}{97} & $E X O C$ & 544 & $\overline{94}$ & 136 & 54 & 431 & 1446 & 3.35 \\
\hline & & & & $D O M$ & 568 & 95 & 142 & 18.2 & 430 & 1443 & 3.36 \\
\hline & & & & $C L I P$ & 532 & 95 & 133 & 18.2 & 426 & 1422 & 3.34 \\
\hline \multirow[t]{3}{*}{ ex5p } & \multirow[t]{3}{*}{8} & 63 & 214 & $E X O C$ & 2058 & 372 & 505 & 889 & 1623 & 4897 & 3 \\
\hline & & & & $D O M$ & 2058 & 373 & 515 & 160 & 1615 & 4853 & 3 \\
\hline & & & & $C L I P$ & 2018 & 372 & 505 & 183.3 & 1611 & 4765 & 2.96 \\
\hline misex 1 & 8 & 7 & $\overline{40}$ & $E X O C$ & 274 & 34 & 69 & 41.1 & 170 & 593 & 3.4 \\
\hline & & & & $D O M$ & 298 & 34 & 75 & 8.8 & 168 & 581 & 3.46 \\
\hline & & & & $C L I P$ & 274 & 34 & 69 & 8.5 & 169 & 574 & 3.4 \\
\hline rd53 & 5 & 3 & 63 & $\overline{E X O C}$ & 72 & 10 & 18 & 7.9 & 30 & 91 & 3.3 \\
\hline & & & & $D O M$ & 72 & 10 & 18 & 1.5 & 30 & 91 & 3.3 \\
\hline & & & & $C L I P$ & 72 & 10 & 18 & 1.8 & 30 & 91 & 3.3 \\
\hline rd73 & 7 & 3 & 274 & $E X O C$ & 148 & 15 & 37 & 64 & 52 & 161 & 3 \\
\hline & & & & $D O M$ & 72 & 10 & 18 & 11.0 & 52 & 161 & 3 \\
\hline & & & & $C L I P$ & 148 & 15 & 37 & 12.1 & 54 & 176 & 3.26 \\
\hline rd84 & 8 & 4 & 515 & $E X O C$ & 248 & $\overline{24}$ & 62 & 213 & 76 & 234 & 3 \\
\hline & & & & $D O M$ & 208 & 25 & 52 & 34.5 & 76 & 234 & 3 \\
\hline & & & & $C L I P$ & 248 & 24 & 62 & 30.6 & 80 & 259 & 3.24 \\
\hline sao2 & 10 & 4 & 133 & $E X O C$ & 436 & 31 & 109 & 2160 & 205 & 1051 & 5.1 \\
\hline & & & & $D O M$ & 540 & 29 & 135 & 49 & 205 & 1051 & 5.1 \\
\hline & & & & $C L I P$ & 436 & 31 & 109 & 48.6 & 191 & 857 & 4.49 \\
\hline squar5 & 5 & 8 & 56 & $\overline{E X O C}$ & 152 & 25 & $\overline{38}$ & 19.2 & 120 & 419 & $\overline{3.49}$ \\
\hline & & & & $D O M$ & 140 & 25 & 35 & 4.4 & 120 & 419 & 3.49 \\
\hline & & & & $C L I P$ & 152 & 25 & 38 & 4.5 & 121 & 426 & 3.52 \\
\hline$\overline{x o r 5}$ & 5 & 1 & 32 & EXOC & 16 & 4 & 4 & 1.4 & 6 & 12 & 2 \\
\hline & & & & $D O M$ & 16 & 4 & 4 & 0.4 & 6 & 12 & 2 \\
\hline & & & & $C L I P$ & 16 & 4 & 4 & 0.4 & 6 & 12 & 2 \\
\hline
\end{tabular}

Table 5.1: A Comparison of the results obtained by running MVGUD with 2 variables in the Bound Set on MCNC Benchmarks 
we conclude that for two variables in the Bound Set it is not worthwhile having an Exact Graph Coloring to calculate the Column Multiplicity in MVGUD, and a good heuristic algorithm to calculate the Column Multiplicity is sufficient.

\subsubsection{A Comparison when $M V G U D$ is run with 4 and 5 variables in the Bound Set}

As the next step $M V G U D$ was run with 4 variables and with 5 variables in the Bound Set. The following tables show the results.

\begin{tabular}{|c|c|c|c|c|c|c|c|c|c|c|c|}
\hline \multicolumn{12}{|c|}{ Comparison of $E X O C, D O M$ and $C L I P$ on MCNC Benchmarks } \\
\hline Benchmark & in & out & cubes & option & $\overline{D F C}$ & $\begin{array}{l}\text { nu of } \\
\text { blocks }\end{array}$ & $\begin{array}{c}\text { Av } \\
\text { Edge\% }\end{array}$ & $\begin{array}{l}\mathrm{T}(\mathrm{s}) \\
(\mathrm{sec})\end{array}$ & NP & $\overline{\mathrm{TC}}$ & $\overline{\mathrm{AC}}$ \\
\hline \multirow[t]{3}{*}{$5 \times \mathrm{p} 1$} & 7 & 10 & 143 & $E X O C$ & 344 & 17 & 62.75 & 2006 & 28 & 123 & 4.4 \\
\hline & & & & CLIP & 344 & 17 & & 29.5 & 28 & 123 & 4.4 \\
\hline & & & & $D O M$ & 344 & 17 & & 29.9 & 28 & 123 & 4.4 \\
\hline \multirow[t]{3}{*}{$9 \mathrm{sym} 1$} & 9 & 1 & 158 & $E X O C$ & 96 & 3 & - & 108 & 11 & 54 & 4.9 \\
\hline & & & & $C L I P$ & 96 & 3 & & 55.2 & 10 & 52 & 5.2 \\
\hline & & & & DOM & 64 & 3 & & 47.3 & 11 & 54 & 4.9 \\
\hline \multirow[t]{3}{*}{ b12 } & 15 & 9 & 172 & $E X O C$ & 284 & 25 & 14.54 & 87 & 130 & 389 & 3 \\
\hline & & & & $C L I P$ & 284 & 25 & & 57.1 & 132 & 387 & 2.9 \\
\hline & & & & $D O M$ & 284 & 25 & & 46.4 & 130 & 389 & 3 \\
\hline \multirow[t]{3}{*}{ bw } & 5 & 28 & 97 & $E X O C$ & 560 & 56 & 55 & 51 & $11 \overline{5}$ & 361 & 3.14 \\
\hline & & & & $C L I P$ & 560 & 56 & & 50.9 & 115 & 361 & 3.14 \\
\hline & & & & $D O M$ & 560 & 56 & & 48.7 & 115 & 361 & 3.14 \\
\hline \multirow[t]{3}{*}{$\overline{e x 5 p}$} & 8 & 63 & 214 & $E X O C$ & - & - & - & - & - & $\checkmark$ & - \\
\hline & & & & $C L I P$ & 2472 & 186 & & 565 & 8 & 35 & 4.4 \\
\hline & & & & $D O M$ & 2472 & 186 & & 516 & 8 & 35 & 4.4 \\
\hline \multirow[t]{3}{*}{ misex1 } & 8 & 7 & 40 & $E X O C$ & 400 & 19 & 60.47 & 318 & 589 & 1831 & 3.1 \\
\hline & & & & $C L I P$ & 388 & 19 & & 24.7 & 587 & 1816 & 3.1 \\
\hline & & & & $D O M$ & 400 & 19 & & 22.5 & 589 & 1831 & 3.1 \\
\hline \multirow[t]{3}{*}{ rd53 } & 5 & 3 & 63 & $E X O C$ & 80 & 6 & 63.5 & 5.4 & 8 & 26 & 3.25 \\
\hline & & & & $C L I P$ & 80 & 6 & & 4.7 & 8 & 26 & 3.25 \\
\hline & & & & $D O M$ & 80 & 6 & & 4.9 & 8 & 26 & 3.25 \\
\hline \multirow[t]{3}{*}{$\mathrm{rd} 73$} & 7 & 3 & 274 & $E X O C$ & 160 & 6 & 71.6 & 46.6 & 10 & 38 & 3.8 \\
\hline & & & & $C L I P$ & 160 & 6 & & 41.6 & 10 & 38 & 3.8 \\
\hline & & & & $D O M$ & 160 & 6 & & 47.2 & 10 & 38 & 3.8 \\
\hline \multirow[t]{3}{*}{$\mathrm{rd} 84$} & 8 & 4 & 515 & $E X O C$ & 220 & 12 & - & 189 & 23 & 75 & 3.3 \\
\hline & & & & $C L I P$ & 220 & 12 & & 127 & 24 & 80 & 3.3 \\
\hline & & & & $D O M$ & 208 & 12 & & 131 & 24 & 75 & 3.3 \\
\hline \multirow[t]{3}{*}{$\mathrm{saO2}$} & 10 & 4 & 133 & $E X O C$ & 416 & 12 & 67 & 423.8 & 52 & 337 & 6.5 \\
\hline & & & & $C L I P$ & 416 & 12 & & 124 & 52 & 337 & 6.5 \\
\hline & & & & $D O M$ & 416 & 12 & & 121 & 52 & 337 & 6.5 \\
\hline \multirow[t]{3}{*}{ squar5 } & 5 & 8 & 56 & $E X O C$ & 200 & 16 & 58.6 & 124.8 & 31 & 94 & 3 \\
\hline & & & & CLIP & 200 & 16 & & 10.2 & 31 & 94 & 3 \\
\hline & & & & $D O M$ & 200 & 16 & & 10.5 & 31 & 94 & 3 \\
\hline \multirow[t]{3}{*}{ xor5 } & 5 & 1 & 32 & $E X O C$ & 20 & 2 & 53 & 1.1 & 2 & 4 & 2 \\
\hline & & & & $C L I P$ & 20 & 2 & & 1.2 & 2 & 4 & 2 \\
\hline & & & & $D O M$ & 20 & 2 & & 1.0 & 2 & 4 & 2 \\
\hline
\end{tabular}

Table 5.2: A Comparison of results obtained by running MVGUD with 4 variables in the Bound Set on MCNC Benchmarks 


\begin{tabular}{|c|c|c|c|c|c|c|c|c|c|c|c|}
\hline \multicolumn{12}{|c|}{ Comparison of EXOC,DOM and $C L I P$ on MCNC Benchmarks } \\
\hline Benchmark & in & out & cubes & option & $\overline{\mathrm{DFC}}$ & $\begin{array}{l}\text { nu of } \\
\text { blocks }\end{array}$ & $\begin{array}{c}\text { Av } \\
\text { Edge\% }\end{array}$ & $\begin{array}{l}\mathrm{T}(\mathrm{s}) \\
(\mathrm{sec})\end{array}$ & $\mathrm{NP}$ & $\mathrm{TC}$ & $\mathrm{AC}$ \\
\hline $5 \times p 1$ & 7 & 10 & 143 & $\begin{array}{l}E X O C \\
C L I P \\
D O M\end{array}$ & $\begin{array}{l}368 \\
368 \\
368\end{array}$ & $\begin{array}{l}18 \\
18 \\
18\end{array}$ & 58.78 & $\begin{array}{c}3240 \\
64 \\
41.8\end{array}$ & $\begin{array}{l}37 \\
37 \\
37\end{array}$ & $\begin{array}{l}136 \\
136 \\
136\end{array}$ & $\begin{array}{l}3.7 \\
3.7 \\
3.7\end{array}$ \\
\hline 9 sym & 9 & 1 & 158 & $\begin{array}{c}\text { EXOC } \\
C L I P \\
D O M\end{array}$ & $\begin{array}{l}128 \\
128 \\
128\end{array}$ & $\begin{array}{l}2 \\
2 \\
2\end{array}$ & 77 & $\begin{array}{l}125 \\
54.6 \\
47.7\end{array}$ & $\begin{array}{l}5 \\
5 \\
5\end{array}$ & $\begin{array}{l}30 \\
30 \\
30\end{array}$ & $\begin{array}{l}6 \\
6 \\
6\end{array}$ \\
\hline $\mathrm{b} 12$ & 15 & 9 & 172 & $\begin{array}{c}E X O C \\
C L I P \\
D O M\end{array}$ & $\begin{array}{l}524 \\
524 \\
524\end{array}$ & $\begin{array}{l}23 \\
23 \\
23\end{array}$ & 46.1 & $\begin{array}{c}383 \\
232 \\
89\end{array}$ & $\begin{array}{c}99 \\
100 \\
98\end{array}$ & $\begin{array}{l}310 \\
307 \\
307\end{array}$ & $\begin{array}{l}3.13 \\
3.07 \\
3.13\end{array}$ \\
\hline $\mathrm{bw}$ & 5 & 28 & 97 & $\begin{array}{c}\text { EXOC } \\
C L I P \\
D O M\end{array}$ & $\begin{array}{l}896 \\
896 \\
896\end{array}$ & $\begin{array}{l}28 \\
28 \\
28\end{array}$ & 55 & $\begin{array}{l}29.1 \\
29.2 \\
29.8\end{array}$ & 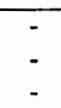 & - & $\begin{array}{l}- \\
- \\
-\end{array}$ \\
\hline ex5p & 8 & 63 & 214 & $\begin{array}{c}E X O C \\
C L I P \\
D O M\end{array}$ & $\begin{array}{c}- \\
3520 \\
3520\end{array}$ & $\begin{array}{l}124 \\
124\end{array}$ & - & $\begin{array}{c}1280.4 \\
724\end{array}$ & $\begin{array}{l}-\overline{-} \\
365 \\
365\end{array}$ & $\begin{array}{c}- \\
1258 \\
1258\end{array}$ & $\begin{array}{l}3.45 \\
3.45\end{array}$ \\
\hline misex1 & 8 & 7 & 40 & $\begin{array}{c}E X O C \\
C L I P \\
D O M\end{array}$ & $\begin{array}{l}432 \\
432 \\
432\end{array}$ & $\begin{array}{l}14 \\
14 \\
14\end{array}$ & 56 & $\begin{array}{l}4400 \\
45.7 \\
23.6\end{array}$ & $\begin{array}{l}35 \\
35 \\
35\end{array}$ & $\begin{array}{l}132 \\
132 \\
132\end{array}$ & $\begin{array}{l}3.77 \\
3.77 \\
3.77\end{array}$ \\
\hline $\operatorname{misex} 2$ & 25 & 18 & 101 & $\begin{array}{c}E X O C \\
C L I P \\
D O M\end{array}$ & $\begin{array}{c}- \\
1152 \\
1152\end{array}$ & $\begin{array}{l}- \\
43 \\
43\end{array}$ & - & $\begin{array}{c}- \\
26665 \\
27540\end{array}$ & 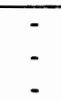 & - & 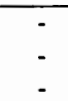 \\
\hline sqrt8 & 8 & 4 & 66 & $\begin{array}{c}E X O C \\
C L I P \\
D O M\end{array}$ & $\begin{array}{l}200 \\
200\end{array}$ & $\begin{array}{l}7 \\
7\end{array}$ & - & $\begin{array}{c}- \\
31 \\
21.3\end{array}$ & - & - & 5 \\
\hline inc & 7 & 9 & 94 & $\begin{array}{c}E X O C \\
C L I P \\
D O M\end{array}$ & $\begin{array}{l}480 \\
480 \\
480\end{array}$ & $\begin{array}{l}18 \\
18 \\
18\end{array}$ & 58.44 & $\begin{array}{c}28620 \\
69 \\
46.9\end{array}$ & - & - & - \\
\hline conl & 7 & 2 & 18 & $\begin{array}{c}E X O C \\
C L I P \\
D O M\end{array}$ & $\begin{array}{l}80 \\
80 \\
80\end{array}$ & $\begin{array}{l}4 \\
4 \\
4\end{array}$ & 71 & $\begin{array}{c}1800 \\
6.5 \\
4\end{array}$ & $\begin{array}{l}8 \\
8 \\
8\end{array}$ & $\begin{array}{l}36 \\
36 \\
36\end{array}$ & $\begin{array}{l}4.5 \\
4.5 \\
4.5\end{array}$ \\
\hline rd73 & 7 & 3 & 274 & $\begin{array}{c}E X O C \\
C L I P \\
D O M\end{array}$ & $\begin{array}{l}200 \\
200 \\
200\end{array}$ & $\begin{array}{l}6 \\
6 \\
6\end{array}$ & 70 & $\begin{array}{l}57.8 \\
51.3 \\
49.6\end{array}$ & $\begin{array}{l}10 \\
10 \\
10\end{array}$ & $\begin{array}{l}36 \\
36 \\
36\end{array}$ & $\begin{array}{l}3.6 \\
3.6 \\
3.6\end{array}$ \\
\hline $\mathrm{rd84}$ & 8 & 4 & 515 & $\begin{array}{c}E X O C \\
C L I P \\
D O M\end{array}$ & $\begin{array}{l}288 \\
288 \\
288\end{array}$ & $\begin{array}{l}8 \\
8 \\
8\end{array}$ & 62.2 & $\begin{array}{l}194 \\
158 \\
159\end{array}$ & $\begin{array}{l}14 \\
14 \\
14\end{array}$ & $\begin{array}{l}54 \\
54 \\
54\end{array}$ & $\begin{array}{l}3.86 \\
3.86 \\
3.86\end{array}$ \\
\hline sao2 & 10 & 4 & 133 & $\begin{array}{c}E X O C \\
C L I P \\
D O M\end{array}$ & $\begin{array}{l}596 \\
596 \\
596\end{array}$ & $\begin{array}{l}12 \\
12 \\
12\end{array}$ & 52 & $\begin{array}{c}3240 \\
231.4 \\
219\end{array}$ & $\begin{array}{l}41 \\
43 \\
41\end{array}$ & $\begin{array}{l}289 \\
287 \\
289\end{array}$ & $\begin{array}{c}7.05 \\
6.7 \\
7.65\end{array}$ \\
\hline squar5 & 5 & 8 & 56 & $\begin{array}{c}\text { EXOC } \\
C L I P \\
D O M\end{array}$ & $\begin{array}{l}256 \\
256 \\
256\end{array}$ & $\begin{array}{l}8 \\
8 \\
8\end{array}$ & 58.6 & $\begin{array}{l}5.6 \\
5.6 \\
5.8\end{array}$ & $\begin{array}{l}- \\
- \\
-\end{array}$ & - & $\begin{array}{l}- \\
-\end{array}$ \\
\hline xor 5 & 5 & 1 & 32 & $\begin{array}{c}E X O C \\
C L I P \\
D O M\end{array}$ & $\begin{array}{l}32 \\
32 \\
32\end{array}$ & $\begin{array}{l}1 \\
1 \\
1\end{array}$ & 53 & $\begin{array}{l}0.5 \\
0.6 \\
0.5\end{array}$ & 5 & - & 5 \\
\hline
\end{tabular}

Table 5.3: A Comparison of results obtained by running $M V G U D$ with 5 variables in the Bound Set on MCNC Benchmarks

In Tables 5.2 and $5.3 A v E d g e \%$ was calculated to see how dense or sparse the graphs generated during the decomposition are. This was calculated in the following way: For any graph with number of nodes $=n$, the total_possible_edges for 
this graph( $100 \%$ edges ) will be equal to $n *(n-1) / 2$. Hence if the number of edges in the graph is equal to $e$, then the edge_percent $=(e * 100) /$ total_possible_edges. This will give the edge_percent in a graph with $n$ nodes and $e$ edges. Since the decomposer calls the function to calculate the Column Multiplicity a number of times, the Av Edge\% was calculated by adding the edge_percent for a graph each time the function to find Column Multiplicity was called, and then dividing this total by the number of times the function to calculate the Column Multiplicity was called.

Table 5.2 shows the comparison of running $M V G U D$ with 4 variables in the Bound Set, and Table 5.3 shows the comparison of running $M V G U D$ with 5 variables in the Bound Set. Looking at the results it is seen that EXOC, DOM and CLIP generate the same results in all the cases in terms of DFC and number of CLB's. The reason for the slow times of MVGUD with EXOC can be explained as follows: When MVGUD is run with 5 variables in the Bound set, in most cases the average number of nodes in the graph is 32 and the edge percentage is always high with the highest being $77 \%$ and the lowest being $46.1 \%$ as shown in Table 5.3 . This means that the graphs generated during decomposition were nearly always (since this is an average) dense graphs. Looking back at Tables 4.2 and 4.3 we see that for these dense graphs EXOC takes a long time to find the Exact solution, hence we have such slow times for EXOC. Also from Tables 4.2 and 4.3 we see that whenever $D O M$ does not generate an exact solution, it is usually 1 or 2 colors away from the Exact solution and rarely more than that, and this being on randomly generated graphs. Now considering that there were 5 variables in the Bound Set, then the Incompatibility graph will have 32 nodes, and for a Curtis decomposition to exist, if a coloring of the graph with 16 colors or less is found then one exists. Now looking at Table 5.3 in the column for Average colors $A C$ it can be seen that the largest average color is 7.65 for the benchmark sao2 But this means that these graphs generated during decomposition, had low chromatic numbers, which were much less than 16 . So even if $D O M$ or CLIP generate a solution that is 2 or 3 colors away, the solution will be accepted as a Curtis De- 
composition because it will still be less than 16 . The same reasoning applies to Table 5.2 where a comparison is made with 4 variables in the Bound Set.

Hence we conclude that for 4 or 5 or greater number of variables in the Bound Set an Exact Graph Coloring does not produce better Curtis Decompositions, and having a good heuristic algorithm to find the Column Multiplicity or even a greedy algorithm to find the Column Multiplicity is good enough.

\subsubsection{A Summary of the Results obtained by testing $D O M, E X O C$ and $C L I P$ on MCNC Benchmarks}

Tables 5.1, 5.2, and 5.3 showed that having an Exact Method of calculating the Column Multiplicity in Functional Decomposition of circuit benchmarks is not necessary. In order to see why the results obtained for MVGUD with different algorithms for calculating the Column Multiplicity were so similar, MVGUD was tested to calculate the size of the difference in the Total number of Colors generated during the entire decomposition by EXOC, CLIP and DOM. But what was found was that, the algorithms EXOC, CLIP and DOM are at some stage of the Decomposition, choosing different Bound Sets as an acceptable decomposition. This is because they are different algorithms and so they are generating different colorings of the incompatibility graphs generated during the decomposition process. Hence this results in different " $\mathrm{G}$ " and " $\mathrm{H}$ " functions, when $M V G U D$ is run with $E X O C$, with $C L I P$, or with $D O M$. This can be seen from the Column "NP" in Tables 5.1, 5.2, and 5.3 which show that depending on which algorithm $E X O C, C L I P$ or DOM is used in $M V G U D$, the number of times the function to calculate the Column Multiplicity is called varies, which means that the Total Colors ("TC") varies, and thus cannot be compared. Hence to conclude this section, what we have proved here is that having an Exact method of calculating the Column Multiplicity for Circuit Benchmarks is not worthwhile in MVGUD for Bound Sets up-to five. Comparisons for Bound Sets greater than five has not been done, because then $E X O C$ is too slow to generate results, for the large graphs involved here, hence not practical. 


\subsection{A Comparison of the different Strategies of finding the Column Multiplicity on Machine Learning Benchmarks}

To see if the same results were obtained on Machine Learning Benchmarks, MVGUD was also tested on Machine Learning Benchmarks. These Benchmarks were obtained from the Wright Labs Database. These are completely specified functions with 8 and 12 variables in the input and one output. Since we were interested in don't-cares in the function the program FLASH(introduced in Chapter 1) was used to convert the above functions into functions with $70 \%$ of don't cares. The following Tables illustrate the results obtained. MVGUD was run in the same way as before, with 2, 4 and 5 variables in the Bound Set.

1. Tables 5.4, 5.5, and 5.6 show the results of running $M V G U D$ on Machine Learning Benchmarks(MLB). These MLB have 8 variables in the Bound Set. In these tables they have $70 \%$ of don't cares. ( cubes $=30 \% * 256$ )

2. Table 5.4 is a result of running $M V G U D$ with 2 variables in the Bound Set.

3. Table 5.5 is a result of running $M V G U D$ with 4 variables in the Bound Set.

4. Table 5.6 is a result of running $M V G U D$ with 5 variables in the Bound Set.

5. On examining these Tables it was seen that here too $E X O C$ fails to improve the quality of Decomposition. The results with all three algorithms prove to be nearly the same, with slight differences in some cases.

6. In Table 5.7, MVGUD was tested on the MLB, 12 variable functions, with the functions being completely specified, and with $70 \%$ of don't cares, and here too there is no change in the results. In Table 5.7 the functions having cubes $=1228$ are fully specified and functions with 410 cubes are with $70 \%$ don't cares.

7. Testing was not done on Bound Sets greater than 5 because for Bound Sets greater than 5, EXOC is too slow to be practical. 


\begin{tabular}{|c|c|c|c|c|c|c|c|c|c|c|}
\hline \multicolumn{11}{|c|}{ Comparison of $E X O C, D O M$ and $C L I P$ on Machine Learning Benchmarks } \\
\hline \multicolumn{2}{|c|}{ Function } & \multicolumn{9}{|c|}{ Algorithm } \\
\hline & & & $\overline{C L I P}$ & & & EXOC & & & $D O M$ & \\
\hline name & cubes & DFC & $\begin{array}{c}\text { nu of } \\
\text { blocks }\end{array}$ & $\mathrm{T}(\mathbf{s})$ & $\overline{\mathrm{DFC}}$ & $\begin{array}{l}\text { nu of } \\
\text { blocks }\end{array}$ & $\mathrm{T}(\mathrm{s})$ & $\overline{\mathrm{DFC}}$ & $\begin{array}{l}\text { nu of } \\
\text { blocks }\end{array}$ & $\mathrm{T}(\mathbf{s})$ \\
\hline addo & 77 & 28 & 5 & 12.5 & 28 & 5 & 24.7 & 28 & 5 & 12.8 \\
\hline add2 & 77 & 28 & 6 & 12.9 & 28 & 5 & 13.1 & 28 & 5 & 13.1 \\
\hline add4 & 77 & 10 & 3 & 7.3 & 10 & 3 & 7.1 & 10 & 3 & 7.6 \\
\hline and or_chain8 & 77 & 22 & 6 & 12 & 22 & 6 & 11.9 & 22 & 6 & 12.5 \\
\hline ch15fo & 77 & 56 & 6 & 20.4 & 68 & 6 & 417 & 68 & 6 & 24.6 \\
\hline ch176fo & 77 & 14 & 4 & 11.7 & 22 & 6 & 13 & 22 & 6 & 14 \\
\hline $\operatorname{ch} 177 \mathrm{fo}$ & 77 & 6 & 2 & 6.3 & 6 & 2 & 6.3 & 6 & 2 & 6.7 \\
\hline $\operatorname{ch} 22 \mathrm{fO}$ & 77 & 28 & 5 & 12 & 28 & 5 & 12.3 & 28 & 5 & 13.4 \\
\hline $\operatorname{ch} 30 f 0$ & 77 & 40 & 5 & 16.4 & 40 & 5 & 15.8 & 40 & 5 & 17.1 \\
\hline $\mathrm{ch} 47 \mathrm{fO}$ & 77 & 28 & 5 & 14.3 & 28 & 5 & 15 & 28 & 5 & 16.4 \\
\hline $\operatorname{ch} 52 f 4$ & 77 & 64 & 5 & 18.5 & 76 & 5 & 19.8 & 76 & 5 & 20.4 \\
\hline $\operatorname{ch} 70 \mathrm{f} 3$ & 77 & 28 & 6 & 14.6 & 28 & 6 & 14.6 & 28 & 6 & 15.8 \\
\hline $\operatorname{ch} 74 \mathrm{f} 1$ & 77 & 52 & 6 & 15 & 44 & 5 & 19.5 & 52 & 6 & 15.5 \\
\hline $\operatorname{ch} 83 \mathrm{f} 2$ & 77 & 64 & 5 & 19.5 & 64 & 4 & 25.7 & 64 & 4 & 22.6 \\
\hline ch8fo & 77 & 22 & 5 & 13.8 & 22 & 5 & 13.8 & 22 & 5 & 15.6 \\
\hline contains_4_one & 77 & 28 & 5 & 19.5 & 44 & 4 & 23.1 & 44 & 4 & 24.1 \\
\hline greater than & 77 & 28 & 7 & 13.3 & 28 & 7 & 13.1 & 28 & 7 & 14.6 \\
\hline intervall & 77 & 44 & 4 & 20.5 & 56 & 5 & 20.3 & 56 & 5 & 21.9 \\
\hline interval2 & 77 & 64 & 4 & 17.2 & 64 & 4 & 21.7 & 64 & 4 & 18.2 \\
\hline kdd1 & 77 & 22 & 6 & 8.9 & 16 & 5 & 8.4 & 16 & 5 & 9.7 \\
\hline $\mathrm{kdd} 2$ & 77 & 22 & 6 & 12.9 & 22 & 6 & 12.3 & 22 & 6 & 14 \\
\hline kdd3 & 77 & 14 & 4 & 7.8 & 14 & 4 & 7.2 & 14 & 4 & 8.9 \\
\hline kdd4 & 77 & 2 & 1 & 6.4 & 2 & 1 & 5.9 & 2 & 1 & 6.9 \\
\hline kdd5 & 77 & 28 & 6 & 12.3 & 28 & 6 & 13.5 & 28 & 6 & $\overline{14.4}$ \\
\hline kdd6 & 77 & 14 & 4 & 8.3 & 14 & 4 & 8.3 & 14 & 4 & 9.2 \\
\hline kdd7 & 77 & 28 & 7 & 10.5 & 28 & 5 & 14.2 & 28 & 5 & 15.4 \\
\hline kdd 8 & 77 & 14 & 4 & 7.1 & 14 & 4 & 6.5 & 14 & 4 & 7.6 \\
\hline kdd 9 & 77 & 28 & 6 & 11.7 & 28 & 7 & 11.7 & 28 & 7 & 12.9 \\
\hline majority gate & 77 & 28 & 6 & 14.5 & 40 & 5 & 16.6 & 40 & 5 & 17.2 \\
\hline modulus2 & 77 & 28 & 6 & 17 & 28 & 5 & 17.3 & 28 & 5 & 18.8 \\
\hline monkish 1 & 77 & 14 & 4 & 8.2 & 14 & 4 & 8.3 & 14 & 4 & 9 \\
\hline monkish2 & 77 & 28 & 6 & 13.9 & 28 & 6 & 13.6 & 28 & 6 & 15 \\
\hline monkish3 & 77 & 22 & 5 & 9.7 & 22 & 5 & 9.5 & 22 & 5 & 10.7 \\
\hline $\operatorname{mux} 8$ & 77 & 46 & 5 & 12.8 & 46 & 5 & 12.2 & 46 & 5 & 13.8 \\
\hline nnr1 & 77 & 64 & 5 & 18.5 & 64 & 5 & 19.7 & 64 & 5 & 19.1 \\
\hline nnr2 & 77 & 14 & 3 & 6.7 & 14 & 3 & 6.7 & 14 & 3 & 7.8 \\
\hline nnr3 & 77 & 88 & 5 & 22.6 & 68 & 4 & 33.2 & 68 & 4 & 26 \\
\hline or_and_chain 8 & 77 & 22 & 6 & 9 & 22 & 6 & 9.2 & 22 & 6 & 10.3 \\
\hline pal & 77 & 22 & 5 & 13.9 & 22 & 5 & 13.4 & 22 & 5 & 14.6 \\
\hline pal_dbl_output & 77 & 92 & 5 & 23.4 & 92 & 6 & 29.1 & 92 & 5 & 25 \\
\hline parity & 77 & 28 & 7 & 11 & 28 & 7 & 11.3 & 28 & 7 & 12.8 \\
\hline primes 8 & 77 & 68 & 5 & 23.3 & 68 & 4 & 26.4 & 68 & 4 & 24.6 \\
\hline remainder 2 & 77 & 68 & 4 & 23.9 & 68 & 4 & 29.8 & 68 & 4 & 25.1 \\
\hline rnd 1 & 77 & 152 & 5 & 23.5 & 152 & 5 & 110 & 152 & 5 & 23.2 \\
\hline rnd2 & 77 & 88 & 4 & 23 & 88 & 4 & 187 & 88 & 4 & 24.9 \\
\hline rnd3 & 77 & 88 & 5 & 20.7 & 88 & 5 & 33.8 & 88 & 4 & 26.1 \\
\hline
\end{tabular}

Table 5.4: A Comparison of results obtained by running $M V G U D$ with 2 variables in the Bound Set on 8 variable MLB 


\begin{tabular}{|c|c|c|c|c|c|c|c|c|c|c|}
\hline \multicolumn{11}{|c|}{ Comparison of $E X O C, D O M$ and $C L I P$ on Machine Learning Benchmarks } \\
\hline \multicolumn{2}{|c|}{ Function } & \multicolumn{9}{|c|}{ Algorithm } \\
\hline & & & CLIP & & & $E \overline{X O C}$ & & & $\overline{D O M}$ & \\
\hline name & cubes & DFC & $\begin{array}{l}\text { nu of } \\
\text { blocks }\end{array}$ & $\mathrm{T}(\mathrm{s})$ & DFC & $\begin{array}{l}\text { nu of } \\
\text { blocks }\end{array}$ & $\mathrm{T}(\mathrm{s})$ & DFC & $\begin{array}{l}\text { nu of } \\
\text { blocks }\end{array}$ & $T(\mathbf{s})$ \\
\hline addo & 77 & 36 & 3 & 11.8 & 36 & 3 & 9.8 & 36 & 3 & 9.7 \\
\hline add2 & 77 & 104 & 3 & 15.6 & 68 & 3 & 12.1 & 68 & $\overline{3}$ & 11.9 \\
\hline add4 & 77 & 18 & 2 & 9.5 & 18 & 2 & 8 & 18 & 2 & 8 \\
\hline and_or_chain8 & 77 & 36 & 3 & 10.6 & 36 & 3 & 9.3 & 36 & 3 & 10.1 \\
\hline $\operatorname{ch} 15 f 0$ & 77 & 104 & 3 & 11.6 & 104 & 3 & 9.4 & 104 & 3 & 11.9 \\
\hline ch176fo & 77 & 36 & 3 & 13 & 36 & 3 & 10.4 & 36 & 3 & 10.4 \\
\hline $\operatorname{ch} 177 \mathrm{fO}$ & 77 & 36 & 3 & 10.2 & 18 & 2 & 7.3 & 18 & 2 & 8.2 \\
\hline $\mathrm{ch} 22 \mathrm{fO}$ & 77 & 36 & 3 & 9.9 & $\overline{36}$ & 3 & 8.3 & 36 & 3 & 9.3 \\
\hline $\operatorname{ch} 30 f 0$ & 77 & 56 & 3 & 12.3 & 56 & 3 & 10.5 & 56 & 3 & 10.7 \\
\hline $\operatorname{ch} 47 \mathrm{fO}$ & 77 & 36 & 3 & 12.4 & 36 & 3 & 11 & 36 & 3 & 11.6 \\
\hline $\operatorname{ch} 52 \mathrm{f} 4$ & 77 & 36 & 3 & 14.1 & 56 & 3 & 11.3 & 56 & 3 & 12.5 \\
\hline $\operatorname{ch} 70 f 3$ & 77 & 36 & 3 & 14.3 & 36 & 3 & 11.4 & 36 & 3 & 10.7 \\
\hline ch.74f1 & 77 & 36 & 3 & 13.9 & 36 & 3 & 10.9 & 36 & 3 & 10.2 \\
\hline $\operatorname{ch} 83 \mathrm{f} 2$ & 77 & 56 & 3 & 14.8 & 56 & 3 & 12 & 56 & 3 & 12 \\
\hline ch8fo & 77 & 36 & 3 & 12.5 & 36 & 3 & 10.9 & 36 & 3 & 10.6 \\
\hline contains_4_one & 77 & 36 & 3 & 12.8 & 36 & 3 & 11.2 & 36 & 3 & 11.3 \\
\hline greater than & 77 & 36 & 3 & 12.1 & 36 & 3 & 9.3 & 36 & 3 & 10.1 \\
\hline interval1 & 77 & 36 & 3 & 13.3 & 36 & 3 & 11.4 & 36 & 3 & 11.1 \\
\hline interval2 & 77 & 80 & 3 & 11.9 & 80 & 3 & 10.5 & 80 & 3 & 9.6 \\
\hline $\mathrm{kdd} l$ & 77 & 12 & 2 & 10.9 & 12 & 2 & 8.8 & 12 & 2 & 8.8 \\
\hline kdd2 & 77 & 36 & 3 & 12.7 & 36 & 3 & 10 & 36 & 3 & 10.5 \\
\hline kdd3 & 77 & 36 & 3 & 11.6 & 36 & 3 & 9.7 & 36 & 3 & 10.1 \\
\hline kdd4 & 77 & 12 & 2 & 5.9 & 12 & 2 & 4.9 & 12 & 2 & 4.6 \\
\hline kdd5 & 77 & 36 & 3 & 10.8 & 36 & 3 & 10.2 & 36 & 3 & 11.2 \\
\hline kdd6 & 77 & 18 & 2 & 7.4 & 18 & 2 & 6 & 18 & 2 & 6 \\
\hline kdd7 & 77 & 36 & 3 & 10.1 & 36 & 3 & 8.1 & 36 & 3 & 8.8 \\
\hline kdd8 & 77 & 12 & 2 & 6.3 & 12 & 2 & 5.2 & 12 & 2 & 5.2 \\
\hline kdd9 & 77 & 36 & 3 & 11.1 & 36 & 3 & 9.2 & 36 & 3 & 9.5 \\
\hline majority gate & 77 & 36 & 3 & 12.5 & 36 & 3 & 10.8 & 36 & 3 & 10.6 \\
\hline modulus2 & 77 & 36 & 3 & 14 & 36 & 3 & 10.5 & 36 & 3 & 10.7 \\
\hline monkish1 & 77 & 18 & 2 & 8.7 & 18 & 2 & 7.6 & 18 & 2 & 7.8 \\
\hline monkish2 & 77 & 36 & 3 & 12.8 & 36 & 3 & 10.4 & 36 & 3 & 9.9 \\
\hline monkish3 & 77 & 36 & 3 & 11.6 & 36 & 3 & 9.9 & 36 & 3 & 10.2 \\
\hline $\operatorname{mux} 8$ & 77 & 36 & 3 & 12.7 & 36 & 3 & 11 & 36 & 3 & 10.1 \\
\hline nnrl & 77 & 36 & 3 & 11 & 36 & 3 & 8.7 & 36 & 3 & 8.8 \\
\hline nnr2 & 77 & 18 & 2 & 6.3 & 18 & 2 & 5 & 18 & 2 & 5.2 \\
\hline $\mathrm{nnr3}$ & 77 & 68 & 3 & 15.7 & 68 & 3 & 11.9 & 68 & 3 & 11.8 \\
\hline or_and_chain 8 & 77 & 36 & 3 & 10.4 & 36 & 3 & 9.3 & 36 & 3 & 9.3 \\
\hline pal & 77 & 36 & 3 & 13.4 & 36 & 3 & 10.9 & 36 & 3 & 10.6 \\
\hline parity & 77 & 36 & 3 & 10.8 & 36 & 3 & 8.9 & 36 & 3 & 9.2 \\
\hline primes8 & 77 & 68 & 3 & 13.8 & 68 & 3 & 12.1 & 68 & 3 & 11.9 \\
\hline remainder2 & 77 & 104 & 3 & 12.4 & 104 & 3 & 9.9 & 104 & 3 & 9.8 \\
\hline rnd1 & 77 & 104 & 3 & 16.1 & 104 & 3 & 236 & 104 & 3 & 11.3 \\
\hline rnd2 & 77 & 104 & 3 & 18 & 104 & 3 & 66 & 104 & 3 & 13.7 \\
\hline rnd3 & 77 & 104 & 3 & 16.3 & 104 & 3 & 660 & 104 & 3 & 12.6 \\
\hline
\end{tabular}

Table 5.5: A Comparison of results obtained by running $M V G U D$ with 4 variables in the Bound Set on 8 variable MLB 


\begin{tabular}{|c|c|c|c|c|c|c|c|c|c|c|}
\hline \multicolumn{11}{|c|}{ Comparison of $E X O C, D O M$ and $C L I P$ on Machine Learning Benchmarks } \\
\hline \multicolumn{2}{|c|}{ Function } & \multicolumn{9}{|c|}{ Algorithm } \\
\hline & & & $\overline{C L I P}$ & & & EXOC & & & $\overline{D O M}$ & \\
\hline name & cubes & $\overline{\mathrm{DFC}}$ & $\begin{array}{l}\text { nu of } \\
\text { blocks }\end{array}$ & $\mathrm{T}(\mathbf{s})$ & $\overline{\mathrm{DFC}}$ & $\begin{array}{l}\text { nu of } \\
\text { blocks }\end{array}$ & $T(\mathbf{s})$ & $\overline{\mathrm{DFC}}$ & $\begin{array}{l}\text { nu of } \\
\text { blocks }\end{array}$ & $\mathrm{T}(\mathbf{s})$ \\
\hline addo & 77 & 48 & 2 & 13.3 & 48 & 2 & 9.3 & 48 & 2 & 9.1 \\
\hline add2 & 77 & 48 & 2 & 12 & 48 & 2 & 11.3 & 48 & 2 & 10.9 \\
\hline add4 & $\overline{77}$ & 48 & 2 & 12 & 48 & 2 & 12.1 & 48 & 2 & 11.5 \\
\hline and_or_chain 8 & 77 & 48 & 2 & 21.7 & 48 & 2 & 13.4 & 48 & 2 & 11.9 \\
\hline ch15fo & 77 & 96 & 2 & 15.4 & 96 & 2 & 11.4 & 96 & 2 & 11.1 \\
\hline $\operatorname{ch} 176 \mathrm{fo}$ & 77 & 48 & 2 & 21.7 & 48 & 2 & 12.3 & 48 & 2 & 12 \\
\hline $\operatorname{ch} 177 \mathrm{fo}$ & 77 & 48 & 2 & 14.2 & 48 & 2 & 9.6 & 48 & 2 & 10.2 \\
\hline $\operatorname{ch} 22 \mathrm{fO}$ & 77 & 48 & 2 & 17.1 & 48 & 2 & 10.8 & 48 & 2 & 10.6 \\
\hline $\operatorname{ch} 30 \mathrm{fo}$ & 77 & 48 & 2 & 19.4 & 48 & 2 & 11.3 & 48 & 2 & 12 \\
\hline $\mathrm{ch} 47 \mathrm{fO}$ & 77 & 96 & 2 & 16.9 & 96 & 2 & 13.3 & 96 & 2 & 13.1 \\
\hline $\operatorname{ch} 52 f 4$ & 77 & 96 & 2 & 21.4 & 96 & 2 & 13.5 & 96 & 2 & 12.4 \\
\hline ch70f3 & 77 & 48 & 2 & 20 & 48 & 2 & 13.1 & 48 & 2 & 13.2 \\
\hline $\operatorname{ch} 74 \mathrm{f} 1$ & 77 & 48 & 2 & 19.2 & 48 & 2 & 12.7 & 48 & 2 & 11.7 \\
\hline $\operatorname{ch} 83 \mathrm{f} 2$ & 77 & 96 & 2 & 17.4 & 96 & 2 & 16.6 & 96 & 2 & 13 \\
\hline $\operatorname{ch} 8 \mathrm{fo}$ & 77 & 48 & 2 & 21.7 & 48 & 2 & 12.1 & 48 & 2 & 12.5 \\
\hline contains 4 -one & 77 & 48 & 2 & 17.1 & 48 & 2 & 11.7 & 48 & 2 & 12.2 \\
\hline greaterthan & 77 & 48 & 2 & 17.1 & 48 & 2 & 11 & 48 & 2 & 10.7 \\
\hline intervall & 77 & 48 & 2 & 17.4 & 48 & 2 & 11.5 & 48 & 2 & 10.9 \\
\hline interval2 & 77 & 96 & 2 & 16.1 & 96 & 2 & 12.1 & 96 & 2 & 14.3 \\
\hline kdd1 & 77 & 8 & 1 & 17.3 & 8 & 1 & 10.1 & 8 & 1 & 11.1 \\
\hline kdd2 & 77 & 48 & 2 & 18.4 & 48 & 2 & 10.4 & 48 & 2 & 11.2 \\
\hline kdd3 & 77 & 48 & 2 & 14.7 & 48 & 2 & 9.8 & 48 & 2 & 9.9 \\
\hline kdd4 & 77 & 8 & 1 & 8.7 & 8 & 1 & 6 & 8 & 1 & 5.4 \\
\hline kdd5 & 77 & 48 & 2 & 16.8 & 48 & 2 & 12.1 & 48 & 2 & 11.8 \\
\hline kdd6 & 77 & 48 & 2 & 21.9 & 48 & 2 & 12 & 48 & 2 & 13 \\
\hline kdd7 & 77 & 48 & 2 & 17 & 48 & 2 & 11.6 & 48 & 2 & 12.1 \\
\hline kdd8 & 77 & 48 & 2 & 18.3 & 48 & 2 & 11.5 & 48 & 2 & 12.6 \\
\hline kdd 9 & 77 & 48 & 2 & 14.2 & 48 & 2 & 10.6 & 48 & 2 & 10.2 \\
\hline majority gate & 77 & 48 & 2 & 17.1 & 48 & 2 & 12 & 48 & 2 & 10.7 \\
\hline modulus2 & 77 & 48 & 2 & 15.1 & 48 & 2 & 10.8 & 48 & 2 & 10.8 \\
\hline monkish1 & 77 & 48 & 2 & 13.9 & 48 & 2 & 9.6 & $\overline{48}$ & 2 & 9.3 \\
\hline monkish2 & 77 & 48 & 2 & 15.7 & 48 & 2 & 12.5 & 48 & 2 & 13.1 \\
\hline monkish3 & 77 & 48 & 2 & 19.2 & 48 & 2 & 11.3 & 48 & 2 & 11.2 \\
\hline $\operatorname{mux8}$ & 77 & 48 & 2 & 15 & 48 & 2 & 10.8 & 48 & 2 & 10.4 \\
\hline nnrl & 77 & 48 & 2 & 16.4 & 48 & 2 & 11.9 & 48 & 2 & 12.4 \\
\hline nnr2 & 77 & 48 & 2 & 17.7 & 48 & 2 & 11.1 & 48 & 2 & 11.5 \\
\hline nnr3 & 77 & 96 & 2 & 17.5 & 96 & 2 & 13.9 & 96 & 2 & 12.4 \\
\hline or and_chain 8 & 77 & 48 & 2 & 18.9 & 48 & 2 & 12.1 & 48 & 2 & 12 \\
\hline pal & 77 & 48 & 2 & 22.3 & 48 & 2 & 11.8 & 48 & 2 & 12.8 \\
\hline parity & 77 & 48 & 2 & 16.4 & 48 & 2 & 11.1 & 48 & 2 & 11.5 \\
\hline primes 8 & 77 & 96 & 2 & 19.4 & 96 & 2 & 13.7 & 96 & 2 & 13.3 \\
\hline remainder2 & 77 & 96 & 2 & 15.9 & 96 & 2 & 10.4 & 96 & 2 & 12 \\
\hline rnd 1 & 77 & 96 & 2 & 17.6 & 96 & 2 & 12.8 & 96 & 2 & 13.8 \\
\hline rnd2 & 77 & 96 & 2 & 18.8 & 96 & 2 & 111 & 96 & 2 & 14.2 \\
\hline Ind3 & 77 & 96 & 2 & 17.8 & 96 & 2 & 14.2 & 96 & 2 & 12.8 \\
\hline
\end{tabular}

Table 5.6: A Comparison of results obtained by running $M V G U D$ with 5 variables in the Bound Set on 8 variable MLB 


\begin{tabular}{|c|c|c|c|c|c|c|c|c|c|c|}
\hline \multicolumn{11}{|c|}{ Comparison of $E X O C, D O M$ and $C L I P$ on Machine Learning Benchmarks } \\
\hline \multicolumn{2}{|c|}{ Function } & \multicolumn{9}{|c|}{ Algorithm } \\
\hline & & & $\overline{C L I P}$ & & & $E X O C$ & & & $\overline{D O M}$ & \\
\hline name & cubes & $\overline{\mathrm{DFC}}$ & $\begin{array}{l}\text { nu of } \\
\text { blocks }\end{array}$ & $T(\mathbf{s})$ & $\overline{\mathrm{DFC}}$ & $\begin{array}{l}\text { nu of } \\
\text { blocks }\end{array}$ & $\mathrm{T}(\mathrm{s})$ & $\overline{\mathrm{DFC}}$ & $\begin{array}{l}\text { nu of } \\
\text { blocks }\end{array}$ & $T(\mathbf{s})$ \\
\hline addo & 1228 & 192 & 8 & 2100 & 192 & 8 & 2100 & 192 & 8 & 2160 \\
\hline addo 90 & 410 & 50 & 7 & 371 & 62 & 8 & 300 & 62 & 8 & 360 \\
\hline add2 & 1228 & 88 & 9 & 2040 & 88 & 9 & 2400 & 88 & 9 & 2100 \\
\hline add2 290 & 410 & 80 & 8 & 360 & 80 & 8 & 360 & 80 & 8 & 420 \\
\hline add 4 & 1228 & 42 & 7 & 1080 & 42 & 7 & 1020 & 42 & 7 & 1140 \\
\hline add $4 \_90$ & 410 & 42 & 7 & 240 & 42 & 7 & 240 & 42 & 7 & 232 \\
\hline contains_4_one & 1228 & 208 & 6 & 3600 & 208 & 6 & 7200 & 208 & 6 & 3600 \\
\hline contains_4_ones_90 & 410 & 164 & 7 & 600 & 164 & 7 & 1180 & 212 & 7 & 600 \\
\hline greater than & 1228 & 216 & 8 & 2760 & 216 & 8 & 4100 & 216 & 8 & 2820 \\
\hline greater_than_90 & 410 & 116 & 9 & 360 & 116 & 9 & 380 & 116 & 9 & 312 \\
\hline interval2 & 1228 & 60 & 7 & 3060 & 60 & 7 & 3500 & 60 & 7 & 3190 \\
\hline interval290 & 410 & 44 & 10 & 480 & 44 & 10 & 560 & 44 & 10 & 420 \\
\hline majority gate & 1228 & 208 & 6 & 3000 & 208 & 6 & 5200 & 208 & 6 & 2700 \\
\hline majority gate_90 & 410 & 260 & 8 & 720 & 260 & 8 & 980 & 260 & 8 & 720 \\
\hline pal & 1228 & 44 & 11 & 2640 & 44 & 11 & $3650-$ & 44 & 11 & 2640 \\
\hline pal_90 & 410 & 90 & 7 & 480 & 90 & 7 & 536 & 90 & 7 & 420 \\
\hline parity & 1228 & $\overline{44}$ & 11 & 1620 & 44 & 11 & 1890 & $\overline{44}$ & 11 & 1680 \\
\hline parity 90 & 410 & 44 & 11 & 360 & 44 & 11 & 364 & 44 & 11 & 378 \\
\hline substr1 & 1228 & 140 & 9 & 2880 & 140 & 9 & 3240 & 140 & 9 & 2880 \\
\hline substr1 190 & 410 & 236 & 7 & 600 & 236 & 7 & 720 & 236 & 7 & 658 \\
\hline subtraction 1 & 1228 & 120 & 8 & 3540 & 120 & 8 & 4890 & 120 & 8 & 3601 \\
\hline subtraction 190 & 410 & 360 & 7 & 600 & 360 & 7 & 730 & 360 & 7 & 600 \\
\hline subtraction3 & 1228 & 316 & 10 & 4620 & - & - & - & 316 & 10 & 3615 \\
\hline subtraction 3.90 & 410 & 312 & 9 & 600 & 312 & 9 & 612 & 312 & 9 & 600 \\
\hline
\end{tabular}

Table 5.7: A Comparison of results obtained by running $M V G U D$ with 2 variables in the Bound Set on 12 variable MLB 
8. Testing was also done to compare the total count of colors generated during the process of decomposition, but here too it was found that the number of times the algorithms for calculating the column multiplicity is called varies for the same function. Hence these Tables are not included here.

\subsubsection{A Summary of the Results Obtained from Testing on Machine Learning Benchmarks}

As can be seen from Tables 5.4, 5.5, 5.6 and 5.7 EXOC was unable to provide a better $D F C$ for the Machine Learning Benchmarks. In order to see the total numbers of colors generated by DOM, EXOC and CLIP on the same graphs, which were generated during the process of Functional Decomposition, the following experiment was performed: $M V G U D$ was made to run with all three algorithms EXOC, CLIP, and DOM calculating the Column Multiplicity, and only the results of one of them was accepted and the results from the other two was discarded. The count of the colors was kept for all three Algorithms, thus demonstrating how EXOC, CLIP, and DOM compare with respect to the total number of colors generated on the same graphs, only now these graphs have been generated from practical function Benchmarks. Table 5.8 shows the result of this comparison. Table 5.8 shows the results of running $M V G U D$ with all three algorithms, DOM, EXOC, and CLIP on the same graphs which were generated during decomposition. Table 5.9 is a summary of the results of Table 5.8. Table 5.9 shows how DOM and CLIP compare with respect to the number of times that the total number of colors generated by DOM and CLIP are the same as the total number of colors generated by EXOC, and the number of times the total colors generated by $D O M$ and $C L I P$ were not exact and by how much.

1. In Table 5.9, the row Exact stands for the case when the total numbers of colors generated by DOM and CLIP was the same as the total colors generated by EXOC. Error 1 stands for the case in which the total numbers of colors generated by $D O M$ and $C L I P$ were one color away from the total 
numbers of colors generated by EXOC, and so on, till Error 6. Corresponding to these rows, the column $N u$ gives the number of times, and column $\%$ is equal to $N u /$ TotalNumberof ProgramRuns $* 100$.

2. As can be seen from the Table 5.9,DOM performs extremely well, and CLIP does not perform so well. DOM thus proves to be a very good heuristic algorithm.

3. Table 5.10 is a total of the rows of Table 5.9 for DOM and CLIP. 


\begin{tabular}{|c|c|c|c|c|c|c|c|c|c|}
\hline \multicolumn{10}{|c|}{$\begin{array}{l}\text { Comparison of the Total Colors generated on Graphs } \\
\text { from Machine Learning } 8 \text { variable Benchmarks }\end{array}$} \\
\hline \multirow[t]{3}{*}{ Benchmark } & \multicolumn{9}{|c|}{ Total Colors } \\
\hline & \multicolumn{3}{|c|}{2 variables in Bound } & \multicolumn{3}{|c|}{4 variables in Bound } & \multicolumn{3}{|c|}{5 variables in Bound } \\
\hline & $D O M$ & $E X O C$ & CLIP & $D O M$ & $E X O C$ & CLIP & $D O M$ & $E X O C$ & $C L I P$ \\
\hline addo & 79 & 79 & 79 & 36 & 36 & 38 & 15 & 15 & 15 \\
\hline add2 & 90 & 90 & 90 & 46 & 45 & 48 & 21 & 21 & 24 \\
\hline add4 & 20 & 20 & 20 & 13 & 13 & 13 & 12 & 12 & 14 \\
\hline and_or_chain8 & 69 & 69 & 69 & 21 & 21 & 22 & 17 & 17 & 18 \\
\hline $\operatorname{ch} 15 \mathrm{fO}$ & 156 & 156 & 158 & 36 & 36 & 38 & 27 & 23 & 24 \\
\hline $\operatorname{ch} 176 \mathrm{fO}$ & 35 & 35 & 36 & 22 & 22 & 23 & 15 & 15 & 15 \\
\hline $\operatorname{ch} 177 \mathrm{fo}$ & 15 & 15 & 15 & 17 & 17 & 17 & 10 & 10 & 11 \\
\hline $\operatorname{ch} 22 \mathrm{fO}$ & 61 & 61 & 61 & 18 & 18 & 18 & 15 & 15 & 15 \\
\hline ch30fo & 126 & 126 & 126 & 33 & 33 & 33 & 22 & 22 & 23 \\
\hline $\operatorname{ch} 47 \mathrm{fO}$ & 111 & 111 & 111 & 33 & 33 & 33 & 27 & 26 & 27 \\
\hline $\operatorname{ch} 52 \mathrm{f} 4$ & 141 & 141 & 142 & 37 & 37 & 40 & 24 & 24 & 25 \\
\hline $\operatorname{ch} 70 f 3$ & 73 & 73 & 73 & 28 & 28 & 28 & 20 & 20 & 20 \\
\hline $\operatorname{ch} 74 \mathrm{f} 1$ & 105 & 105 & 105 & 26 & 26 & 29 & 25 & 25 & 25 \\
\hline $\operatorname{ch} 83 f 2$ & 123 & 123 & 123 & 44 & 44 & 45 & 28 & 28 & 29 \\
\hline $\operatorname{ch} 8 \mathrm{fo}$ & 90 & 90 & 90 & 21 & 21 & 21 & 17 & 17 & 18 \\
\hline contains_4_ones & 126 & 126 & 126 & 32 & 32 & 34 & 24 & 23 & 25 \\
\hline greater than & 100 & 100 & 100 & 35 & 35 & 35 & 20 & 20 & 21 \\
\hline interval 1 & 134 & 134 & 135 & 31 & 31 & 32 & 18 & 17 & 19 \\
\hline interva!2 & 123 & 123 & 124 & 35 & 35 & 36 & 30 & 30 & 32 \\
\hline kdd 1 & $\overline{46}$ & 46 & 46 & 19 & 19 & 19 & 12 & 12 & 12 \\
\hline kdd2 & 58 & 58 & 58 & 18 & 18 & 18 & 12 & 12 & 12 \\
\hline kdd3 & 38 & 38 & 38 & 23 & 23 & 23 & 13 & 13 & 13 \\
\hline $\mathrm{kdd} 4$ & 16 & 16 & 16 & 7 & 7 & 7 & 3 & 3 & 3 \\
\hline kdd5 & 89 & 89 & 89 & 31 & 31 & 32 & 20 & 20 & 24 \\
\hline kdd6 & 29 & 29 & 29 & 9 & 9 & 9 & 12 & 12 & 12 \\
\hline kdd7 & 59 & 59 & 59 & 24 & 24 & 25 & 21 & 20 & 21 \\
\hline kdd8 & 30 & 30 & 30 & 11 & 11 & 11 & 13 & 13 & 14 \\
\hline kdd9 & 77 & 77 & 77 & 27 & 27 & 27 & 18 & 18 & 20 \\
\hline majority gate & 94 & 94 & 94 & 32 & 32 & 32 & 21 & 21 & 23 \\
\hline modulus2 & 118 & 118 & 118 & 36 & 36 & 39 & 20 & 20 & 22 \\
\hline monkish 1 & 31 & 31 & 31 & 14 & 14 & 15 & 12 & 11 & 13 \\
\hline monkish2 & 84 & 84 & 85 & 34 & 34 & 37 & 23 & 23 & 25 \\
\hline monkish3 & 73 & 73 & 73 & 28 & 28 & 29 & 17 & 17 & 17 \\
\hline $\operatorname{mux} 8$ & 102 & 102 & 102 & 38 & 38 & 38 & 19 & 19 & 22 \\
\hline $\mathrm{nnr} 1$ & 131 & 131 & 134 & 27 & 27 & 30 & 26 & 26 & 30 \\
\hline $\mathrm{nnr2}$ & 36 & 36 & 36 & 11 & 11 & 11 & 15 & 15 & 15 \\
\hline nnr3 & 187 & 187 & 189 & 42 & 42 & 45 & 31 & 31 & 34 \\
\hline or_and_chain8 & 56 & 56 & 56 & 24 & 24 & 24 & 15 & 15 & 16 \\
\hline pal & 74 & 74 & 74 & 23 & 23 & 24 & 14 & 14 & 14 \\
\hline pal_dbl_output & 219 & 219 & 220 & 43 & 43 & 46 & 33 & 31 & 37 \\
\hline parity & 46 & 46 & 46 & 18 & 18 & 18 & 12 & 12 & 14 \\
\hline primes 8 & 124 & 124 & 124 & 36 & 36 & 37 & 27 & 27 & 28 \\
\hline remainder2 & 157 & 157 & 159 & 34 & 34 & 35 & 24 & 24 & 29 \\
\hline rnd l & 205 & 205 & 207 & 49 & 49 & 50 & 34 & 34 & 39 \\
\hline rnd2 & 215 & 215 & 216 & 54 & 54 & 56 & 37 & 37 & 42 \\
\hline rnd3 & 156 & 156 & 160 & 52 & $\overline{52}$ & 54 & 33 & 32 & 34 \\
\hline
\end{tabular}

Table 5.8: A Comparison of the Total Colors obtained by running MVGUD on Machine Learning Benchmarks 


\begin{tabular}{|c|c|c|c|c|c|c|c|c|c|c|c|c|}
\hline \multicolumn{13}{|c|}{ Summary of Results of Comparison of the Total Colors } \\
\hline \multicolumn{13}{|c|}{ Total Number of Program Runs $=46$} \\
\hline & \multicolumn{6}{|c|}{$D O M$} & \multicolumn{6}{|c|}{ CLIP } \\
\hline & \multicolumn{2}{|c|}{ Bound $=2$} & \multicolumn{2}{|c|}{ Bound $=4$} & \multicolumn{2}{|c|}{ Bound $=5$} & \multicolumn{2}{|c|}{ Bound $=2$} & \multicolumn{2}{|c|}{ Bound $=4$} & \multicolumn{2}{|c|}{ Bound $=5$} \\
\hline & $\overline{\mathrm{Nu}}$ & $\%$ & $\mathrm{Nu}$ & $\%$ & $\mathrm{Nu}$ & $\%$ & $\mathrm{Nu}$ & $\%$ & $\mathrm{Nu}$ & $\%$ & $\mathrm{Nu}$ & $\%$ \\
\hline Exact & $\overline{46}$ & 100 & 45 & 97.8 & 41 & 89.1 & 32 & 66.1 & 20 & 43.5 & 14 & 30.5 \\
\hline Error 1 & - & - & 1 & 2.1 & 3 & 6.5 & 8 & 17.4 & 13 & 28.3 & 11 & 23.9 \\
\hline Error 2 & - & - & - & - & 1 & 2.1 & 4 & 8.6 & 5 & 10.8 & 12 & 26.1 \\
\hline Error 3 & - & - & - & - & - & - & 1 & 2.1 & 8 & 17.4 & 3 & 6.5 \\
\hline Error 4 & - & - & - & - & 1 & 2.1 & 1 & 2.1 & - & - & 3 & 6.5 \\
\hline Error 5 & - & - & - & - & - & - & - & - & - & - & 2 & 4.3 \\
\hline Error 6 & - & - & - & - & - & - & - & - & - & - & 1 & 2.1 \\
\hline
\end{tabular}

Table 5.9: A Comparison of Total Colors generated by DOM, and CLIP compared with total colors generated by $E X O C$ on the same graphs for two, four and five variables in the Bound Set for Machine Learning Benchmarks

\begin{tabular}{|c|c|c|c|c|}
\hline \multicolumn{5}{|c|}{ Sum of Table 1 } \\
\hline \multicolumn{4}{|c|}{ Total Number of Program Runs $=\mathbf{1 3 8}$} \\
\cline { 2 - 5 } & \multicolumn{2}{|c|}{ DOM } & \multicolumn{2}{c|}{ CLIP } \\
\hline Total Number & Total \% & Total Number & Total \% \\
\hline Exact & 132 & 95.6 & 66 & 47.8 \\
\hline Error 1 & 4 & 2.8 & 33 & 23.9 \\
\hline Error 2 & 1 & 0.7 & 21 & 15.2 \\
\hline Error 3 & - & - & 12 & 8.7 \\
\hline Error 4 & 1 & 0.7 & 4 & 2.8 \\
\hline Error 5 & - & - & 2 & 1.4 \\
\hline Error 6 & - & - & 1 & 0.7 \\
\hline
\end{tabular}

Table 5.10: A Summary showing the Addition of the Total Colors obtained in Table 5.9 


\subsection{Some Questions and Conclusions Reached from the Results of the Testing}

We believe that to solve any problem it is important to go deep into the problem and investigate the problem carefully. Here the problem for us was Functional Decomposition. Functional Decomposition has always been a very complex problem, and all the research done till date just tries to solve the problem more efficiently without trying to reason why Functional Decomposition is such a difficult problem. Here we have investigated only the part of Functional Decomposition which involves finding the Column Multiplicity, but the results obtained in this Chapter provide a very deep insight into the Column Multiplicity part of Functional Decomposition. By the results of the testing we can definitely say that we have proved that an Exact Graph Coloring is not required to find the Column Multiplicity where Curtis Decompositions are considered. Exact Graph Colorings only take up more time and fail to produce any significant change in the results. This is true with respect to both Circuit Benchmarks and Machine Learning Benchmarks.

Also the results shown in the Summary Tables 5.9 and 5.10 raise the question that in cases where CLIP did not generate the same total numbers of colors as EXOC, why did the DFC not improve when we used EXOC? The only possible answer to this question is that the decompositions generated by CLIP were still acceptable decompositions, even if they use non minimum numbers of colors which in turn means that these graphs generated during the decomposition process must be having low chromatic numbers. This provides a very valuable insight into the kinds of graphs that are generated during the decomposition process. This tells us that the graphs generated during the decomposition process are definitely of a different nature than random graphs. Which raises another question: Why are the graphs generated during the decomposition process different from the graphs generated randomly? This question is beyond the scope of this thesis, but it is a very interesting question, because answering this question 
might provide a better insight into the Functional Decomposition process.

\subsection{What is the next step to solve the Column Multiplicity Problem}

In this Chapter the heuristic graph coloring program (DOM), the exact graph coloring program (EXOC) and the clique partitioning program (CLIP) were tested in decomposition and the results proved that an Exact Graph Coloring does not yield better Curtis Decompositions. Now we know that there are basically four methods to find the Column Multiplicity, namely: Clique Partitioning, Clique Covering, Graph Coloring and Set Covering. Out of these methods the Clique Partitioning and Graph Coloring have been investigated by us. Hence what was needed now was to try either a Clique Covering or a Set Covering to see if they can improve the quality of decompositions achieved. Clique Covering and Set Covering are both similar, a Clique Covering is done on a compatibility graph while a Set Covering is done on a table. Exactly analogous to a Clique Covering is a Multi-Coloring. The only difference being that a Multi-Coloring is done on an incompatibility graph. Hence it was needed to test one of these methods in the Decomposition process. Since we had already written two Graph Coloring programs, it was decided to write a Multi-Coloring program in order to test the Column Multiplicity problem in Functional Decomposition further. MultiColoring, and the relation between a Multi-Coloring and a Clique-Covering is explained in more detail in the next Chapter. 


\section{CHAPTER 6}

\section{A NEW APPROACH TO MULTI-COLORING USING DOMINATIONS: COLUMN COMPATIBILITY FOR FUNCTIONS AND RELATIONS}

This Chapter begins with an introduction of some basic Notions and Definitions, because before the different sections in this Chapter are introduced, it is necessary to understand these notions and definitions.

\subsection{Basic Notions and Definitions}

\subsubsection{Multi-Coloring}

Definition 6.1 A multi-coloring of a graph is assigning a node with as many colors as possible, without creating a conflict between any two nodes, meaning, the sets of colors assigned to any two adjacent nodes are disjoint.

\subsubsection{Maximum Independent Sets in an Incompatibility Graph}

Definition 6.2 A Maximum Independent Set in an incompatibility graph is a maximum set of nodes in the incompatibility graph which can be given the same color.

6.2 A Comparison showing the Equivalence of a Multi-Coloring and a Maximum Clique Covering

Figure 6.1 shows a comparison between a Maximum Clique Covering and a Multi-Coloring. Figure 6.1(a) is the incompatibility Graph with the multi-colors assigned. Figure 6.1(b) shows the same realization of the function, only now it is a 


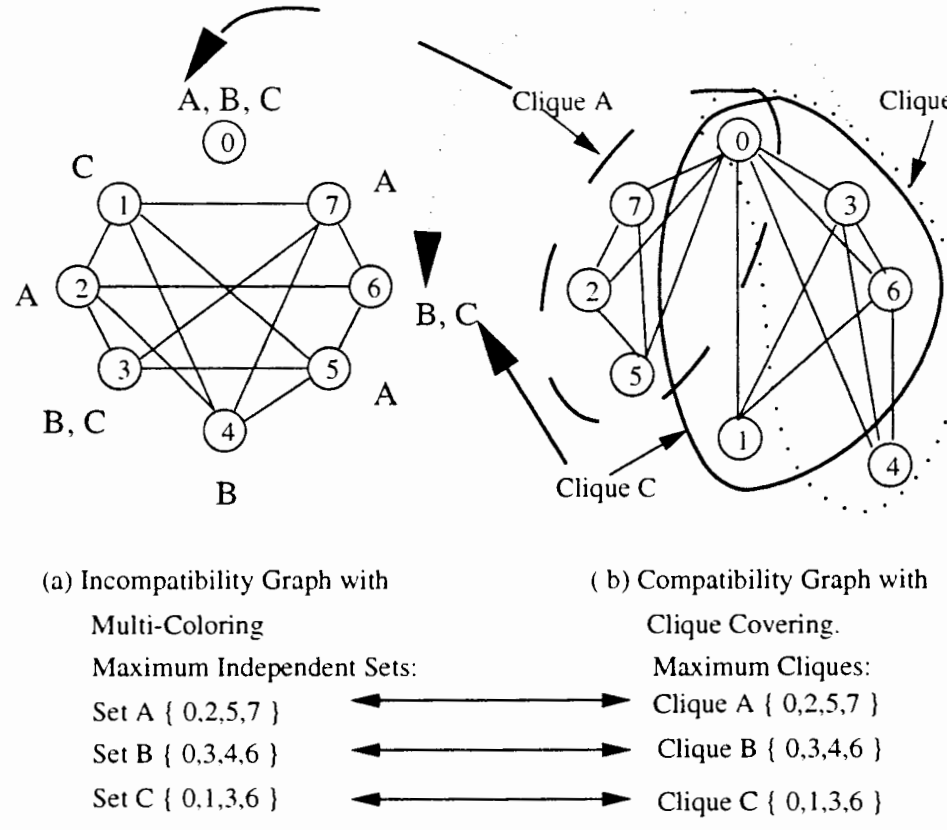

Figure 6.1: Difference between Clique Covering and Multi-Coloring

Compatibility Graph. From Figure 6.1(b) it can be seen that the Clique Covering produced 3 cliques. The same information was obtained from the multi-coloring, shown in Figure 6.1(a). Also it can be seen from Figure 6.1, that in Figure 6.1(a) the Maximum Independent Set A in the incompatibility graph is the same as Clique $\mathrm{A}$ in the compatibility graph in Figure 6.1(b). Similarly, Maximum Independent Set B is the same as Clique B, and the Maximum Independent Set C is the same as Clique $\mathrm{C}$.

\subsection{Why it was decided to use a Multi-Coloring in Decomposition}

This thesis started of with the testing of the Column Multiplicity problem in Functional Decomposition, and in Chapter 5, it was proved that having an Exact Graph Coloring to solve the problem of Column Multiplicity in Functional Decomposition does not improve the DFC. Hence this meant that maybe what is needed is a Multi-Coloring (A Multi-Coloring is functionally equivalent to a Clique Covering as shown in Section 6.2), to solve the problem of Column Multi- 
plicity in Functional Decomposition. Now as can be seen from Section 6.1.1, for a Multi-Coloring or Clique-Covering to improve the quality of decompositions achieved, the decomposer must make use of the overlapping independent sets. At the same time Dr Perkowski developed the new concept of Relations, and MVGUD was extended so that it now had the ability to decompose Relations too. This new concept of Relations provided the ability to take advantage of the overlaps obtained by the Multi-Coloring, thus with Relations the Multi-Coloring could be properly tested and evaluated. In the next Section Multi-Valued Relations and their applications will be introduced. This is the application in which we will show how the Multi-Coloring can improve the quality of the Curtis decompositions achieved. Then the details of the Multi- Coloring program are introduced. In the next Chapter an evaluation of how the Multi-Coloring performs on random graphs is presented. An Analysis of the Multi-Coloring on graphs generated during the decomposition process is also given in the next Chapter.

\subsubsection{An Introduction to Multi-Valued Relations}

Until very recently, logic synthesis focussed on efficient methods and algorithms to optimize the hardware of digital computers and other digital circuits. In the last few years an increased trend has occurred to apply the logic synthesis methods also in image processing, machine learning, knowledge discovery, database optimization, AI, image coding, automatic theorem proving and verification of software and hardware. What was found was that these Machine Learning Functions have quite different properties than Circuit Functions(MCNC). The characteristics of the Machine Learning data is:

1. Machine Learning Functions have a very high number of don't cares, typically more than $99 \%$.

Definition 6.3 A don't care is defined as a combination of argument values for which the function value is not specified. 
In the Machine Learning community don't cares are referred to as unknown.

2. Most Machine Learning Functions are Multi-Output.

3. Practical Machine Learning problems require at least 30 binary input variables but more typically, about 100 multi-valued variables. As the number of input and output variables increases there is only a small increase in the number of positive and negative samples but a dramatic increase in the number of don't cares.

4. Arguments (variables) in Machine Learning problems are naturally multiplevalued (or continuous and then discretized).

5. In the context of Machine Learning when one talks of "noise", one means a situation when there is some data that was classified correctly, but then noise causes it to change its value. Another situation is when the value of a minterm is or becomes a don't care. This would be referred to as an unknown in the Machine Learning community. This is however not a very good idea and some information is lost in the preprocessing of the data for the Machine Learning program. In [22, 23, 24, 25] a more general solution has been proposed. Let us assume that the choices for a three valued decision variable $X$ are: "a car", "a tank", and "an airplane", denoted as values $X^{0}, X^{1}$, and $X^{2}$ respectively. Then a standard don't care, I do not know, would correspond to $X^{0,1,2}$, denoted in a standard way by "-". But a decision saying "I do not know but not an airplane" would be a relation denoted by $X^{0,1}$. This is called a generalized don't care. If a function has generalized don't cares in the output then it is no longer called a function, now it is called a relation.

Table 6.1 shows a multi-valued relation with four binary inputs and one five valued output. In a function the output can only have one value, but as shown in Table 6.1 in row 0 , minterm 0000 maps to the output $f=0$ or 1 . Therefore this row specifies a generalized don't care. This says that for input minterm 0000 


\begin{tabular}{|c|cccc|c|}
\hline row_no & $\mathrm{a}$ & $\mathrm{b}$ & $\mathrm{c}$ & $\mathrm{d}$ & $\mathrm{f}$ \\
\hline 0 & 0 & 0 & 0 & 0 & 0,1 \\
1 & 0 & 1 & 0 & 0 & 1,2 \\
2 & 1 & 1 & 0 & 0 & 0 \\
3 & 0 & 0 & 0 & 1 & 0,3 \\
4 & 1 & 1 & 0 & 1 & 0,3 \\
5 & 1 & 0 & 0 & 1 & 0,4 \\
6 & 1 & 0 & 0 & 0 & 0,3 \\
7 & 0 & 0 & 1 & 1 & 1,3 \\
8 & 0 & 1 & 1 & 1 & 0,1 \\
9 & 0 & 0 & 1 & 0 & 2,3 \\
10 & 1 & 0 & 1 & 0 & 1,4 \\
11 & 0 & 1 & 1 & 0 & 2,3 \\
\hline
\end{tabular}

Table 6.1: Table showing Relations in a four binary input and one multi-output Function

the output $f$ can be a 0 or a 1 but not both. The designer has the freedom to select any one of these values, whichever simplifies the final description more. In this Table if we had a row with all five values $f^{0,1,2,3,4,5}=0,1,2,3,4,5$, then this would correspond to a classical don't care. If there is only a subset of values for example $f^{0,1}$, then this specifies a a generalized don't care. To better understand this assume that the meaning of the values of the output decision $f$ stand for: 0 - a bicycle, 1 - a motorcycle, 2 - a car, 3 - a bus, 4 - a train, and 5 - an airplane. Then the position 0,1 in the output $f$ in row 1 will stand for either a bicycle or a motorcycle, which means something is known but what is known is not precise. Here a value $0,1,2,3,4,5$ would stand for a complete unknown. This corresponds to a standard don't care for functions. More details on relations and decomposition of Multi-Valued Relations can be obtained from [22, 23, 24, 25]. 


\subsubsection{An Example showing Decomposition of Multiple-Valued Rela- tions}

In Figure 6.2 an example is shown which illustrates how to perform decompositions of Multi-Valued Relations. Figure 6.2(a) shows the Karnaugh map of a relation having four inputs and one 5 valued output. From this Karnaugh map a compatibility graph is created, where the nodes of the compatibility graph are a direct mapping of the columns of the Karnaugh map.

Definition 6.4 Two columns of a Karnaugh map of a single output Multi-Valued Relation with generalized don't cares in the output are compatible if each entry in the two columns, has at least one value in common.

Thus the compatibility graph is created. Figure $6.2(\mathrm{~b})$ shows the compatibility graph. In Figure 6.2(b) the columns in brackets near the edges between two nodes $C_{i}$ and $C_{j}$ represents the combined column $C_{i j}$. One important point to be made here is: When there are generalized don't cares in the Karnaugh map, the checking of Compatibility of columns is different than the check for incomplete tautology defined earlier. This is because if Columns $\mathrm{C} 0, \mathrm{Cl}$, and $\mathrm{C} 2$ are pairwise compatible, it does not mean that they are compatible as a clique. From the compatibility graph the Cliques $\{\mathrm{B} 0, \mathrm{~B} 1\}$ and $\{\mathrm{B} 1, \mathrm{~B} 2, \mathrm{~B} 3\}$ are obtained. The colors assigned are:

Column B0 : Color A,

Column B1 : Color A,B,

Column B2: Color B,

Column B3: Color B.

Then Column B0 is given the code $v=0$, Columns B2 and B3 are given the code $v=1$, and column $\mathrm{B} 1$ is given the code 0 and code 1 . From this the maps of relations $\mathrm{G}$ and $\mathrm{H}$ are built. Thus this explains a disjoint Curtis decomposition of a relation $F$, into $G$ and $H$ relations. 


\begin{tabular}{|c|c|c|c|c|}
\hline \multicolumn{2}{|c|}{$\mathrm{cd} \mathrm{B}^{\mathrm{B}}$} & $\mathrm{B} 1$ & B2 & B3 \\
\hline & 00 & 01 & 11 & 10 \\
\hline 00 & 0,2 & 0,3 & 1,3 & 2,3 \\
\hline 01 & 1,2 & & 0,1 & 1,3 \\
\hline 10 & 0 & 0,3 & - & - \\
\hline 11 & 0,3 & 0,4 & - & 1,4 \\
\hline
\end{tabular}

(a) Karnaugh map of Function $\mathrm{F}$

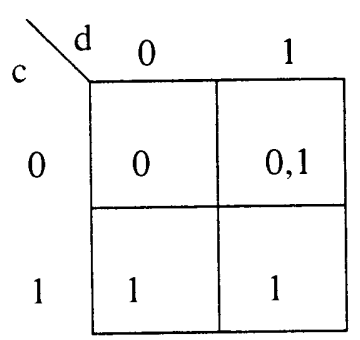

(c) G function with Relations

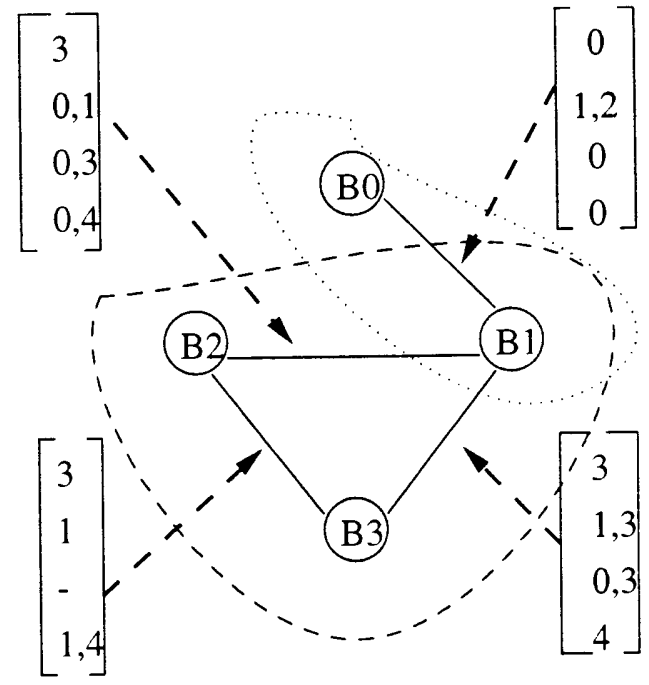

(b) Compatibility graph of Function F.

\begin{tabular}{r|l|l|}
\multicolumn{1}{c|}{$\mathrm{ab}$} & $\mathrm{g}$ & \\
00 & 0 & 1 \\
\cline { 2 - 3 } & 0 & 3 \\
\cline { 2 - 3 } & 1,2 & 1 \\
\hline 11 & 0 & 0,3 \\
\cline { 2 - 3 } 10 & 0 & 4 \\
\hline
\end{tabular}

(d) H function with Relations

Figure 6.2: An Example showing Decomposition of Multiple-Valued Relations 


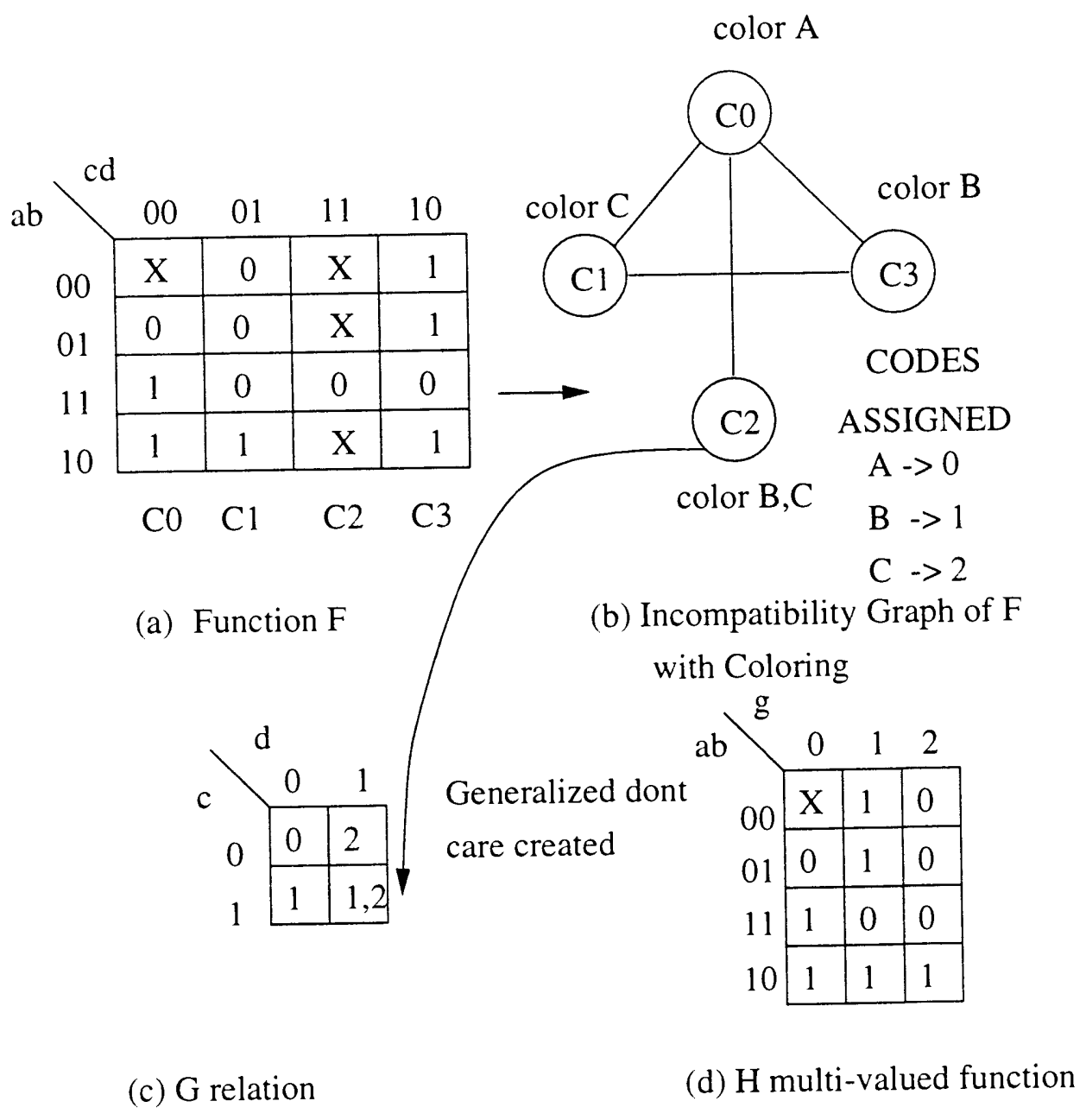

Figure 6.3: An Example showing Decomposition of a Binary Function and the Creation of Relations

\subsubsection{An Example showing Decomposition of a Binary Function and the creation of Relations}

In the example shown in Figure 6.3 it is shown how relations can be created from a Function by using a Multi-Coloring program to find the column multiplicity. Figure 6.3(a) shows a function with four inputs and one output. Figure 6.3(b) shows the incompatibility graph created from the Karnaugh map. Since this is a function, the checking of the compatibility of columns is a check for an incomplete tautology. On doing a multi-coloring of the incompatibility graph it is found that 


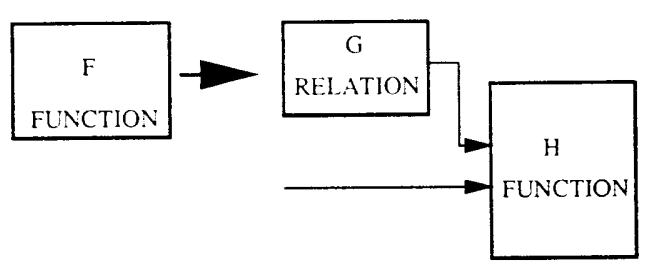

(a) Initial Function $\mathrm{F}$ (b) Initial Step of

Decomposition

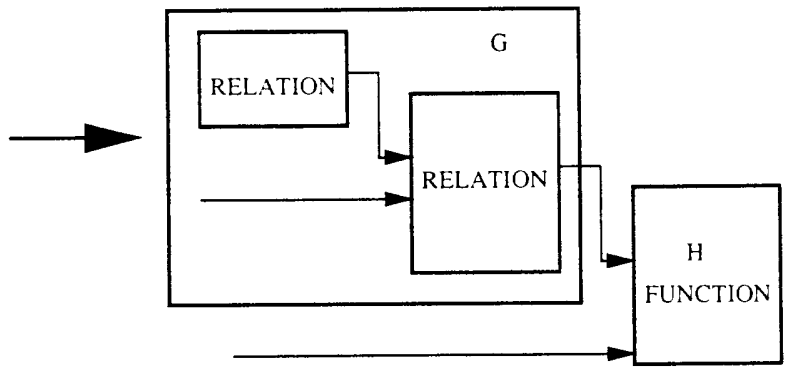

(c) Second Step of

Decomposition

Figure 6.4: Decomposing a Function and using a Multi-Coloring to produce G Relations and $\mathrm{H}$ functions

column $\mathrm{C} 2$ can have two possible colors. The final colors assigned are:

Color A: $\{\mathrm{C} 0\}$,

Color B: $\{\mathrm{C} 2, \mathrm{C} 3\}$.

Color C: $\{\mathrm{C} 1, \mathrm{C} 2\}$.

Column $\mathrm{C} 0$ is given the code $v=0$, column $\mathrm{C} 1$ is given the code $v=2$, column $\mathrm{C} 3$ is given the code $v=1$, and column $\mathrm{C} 2$ is given the codes $v=1,2$. Thus as a result of creating the $\mathrm{G}$ block, a multi-valued generalized don't care is obtained, as shown in Figure 6.3(c). But since the $\mathrm{H}$ block is formed from the original Karnaugh map from Figure 6.3(a) (which is a function), the $\mathrm{H}$ block will be a function and not a relation. But as can be seen from Figure 6.3(d) now the $\mathrm{H}$ block is a multi-valued function. Since this $\mathrm{H}$ function will be used for the next level of decomposition, in the next level too the $\mathrm{H}$ block cannot be a relation.

From the examples shown in 6.3.2 and 6.3.3 the following theorems can be formulated:

Theorem 6.1 If a function $\mathbf{F}$ is being decomposed, and if a Multi-Coloring is used to find the column multiplicity the $G$ block can be a relation but the $H$ block will always be a function.

Proof 6.1 In order to perform a Curtis Decomposition of a function, the $G$ block is created from the encodings given to the intermediate variables. Thus if 
a Multi-Coloring is used to find the column multiplicity, then a column can have more than one code, which will result in generalized don't cares in the $G$ block, thus making the block $G$ a relation. But the $H$ block is created directly from the columns of the Karnaugh map of the original function(explained in Chapter 2, Section 2.4), and since the original input is a function, the $H$ block cannot be a relation. Now as this new $H$ block is used for the next step of the decomposition process, in the next step of decomposition too the $H$ block will not be a relation. The only possible situation when the $H$ block can be a relation is if a decomposed $G$ block which is a relation is decomposed further, but this means that the input is a relation. This proves Theorem 6.1.

Figure 6.4 illustrates Theorem 6.1. Figure 6.4(a) shows a Function, which is decomposed using a Multi-Coloring to find the Column Multiplicity, resulting in a $\mathrm{G}$ relation and an $\mathrm{H}$ function in Figure 6.4(b). The $\mathrm{H}$ function in Figure 6.4(b) is decomposed next, so also in the next step of decomposition the $\mathrm{G}$ block is a relation but the $\mathrm{H}$ block is a function (as shown in Figure 6.4(c)).

Theorem 6.2 If a relation $\mathbf{F}$ is being decomposed, the $H$ block can be a relation, if a graph coloring is used then the $G$ block will be a function, but if a Multi-Coloring is used then only the $G$ block can be a relation.

Proof 6.2 Now the input is a relation. As the $H$ block is created from the columns of the original Karnaugh map (explained in Chapter 2, Section 2.4), which in this case specifies a relation, if the combination of compatible columns in the Karnaugh map leads to a column with generalized don't cares, the $H$ block will be a relation. When creating the $G$ block the original Karnaugh map is not used, instead the encodings chosen for the intermediate variables are used to create the G block(explained in Chapter 2, Section 2.4). Thus if a column does not have more than one code, then the $G$ block cannot have any generalized don't cares, and a column can have more than one code only if a Multi-Coloring or a Clique Covering is used to find the column multiplicity, thus proving Theorem 6.2. 


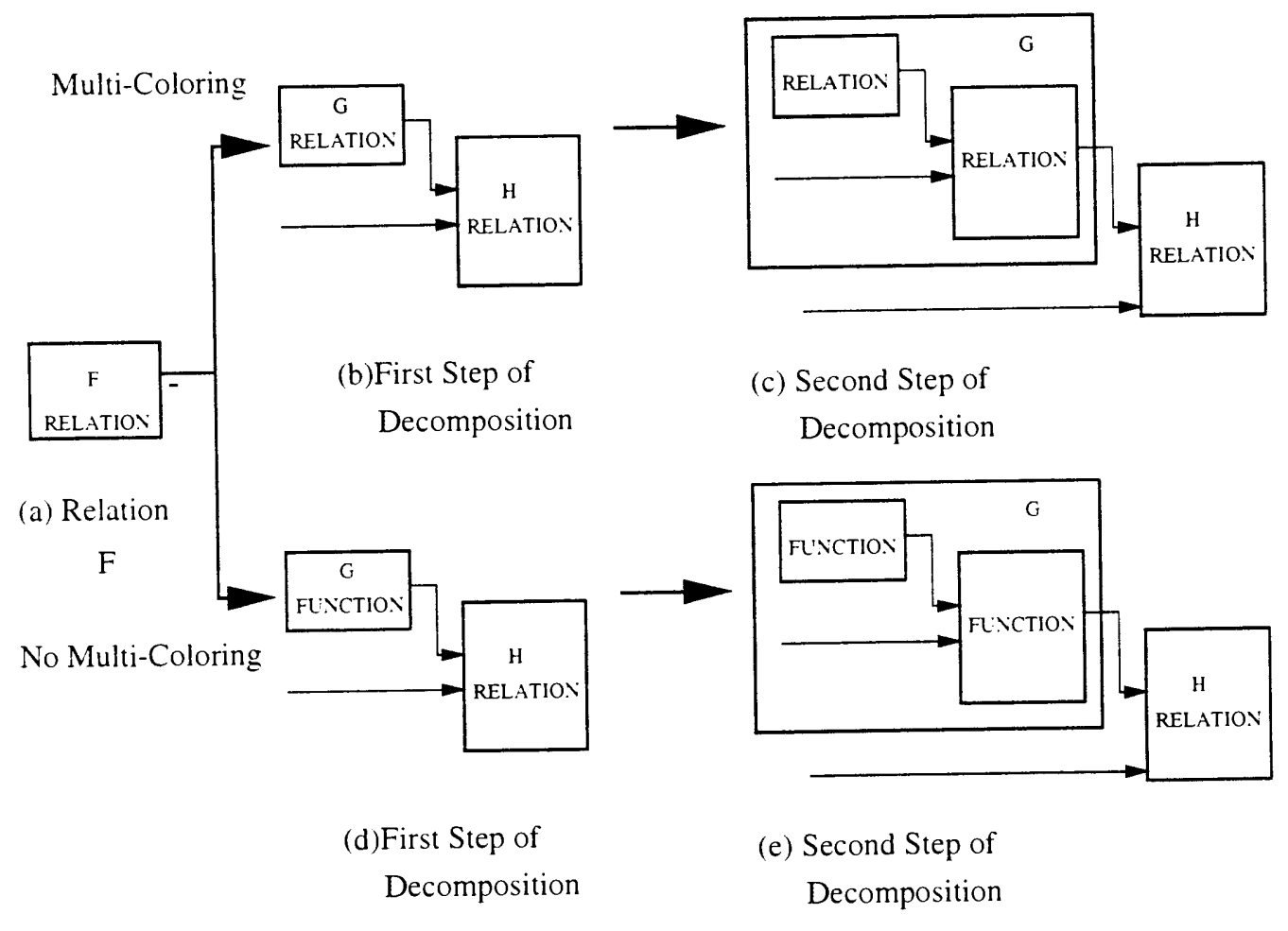

Figure 6.5: The Difference Decompositions obtained by using a Graph Coloring or a Multi-Coloring while Decomposing a Relation 
Figure 6.5(a) shows a Relation. Figure 6.5(b) and Figure 6.5(c) shows the resulting decomposition if a Multi-Coloring is used to find the column multiplicity, and Figure 6.5(d) and Figure 6.5(e) present the resulting decomposition when a graph coloring is used. As can be seen, when a Multi-Coloring is used both the $\mathrm{G}$ and $\mathrm{H}$ blocks can be relations while when using a Graph Coloring only the $\mathrm{H}$ block can be a relation.

The advantage of having relations is that now the Functional Decomposer has the freedom to select any of the values specified for a generalized don't care, whichever simplifies the final description more. Thus the more the generalized don't cares in the relation, the more is the freedom to find the decomposition of the lowest cost.

Relations occur in various applications in Machine Learning, and Knowledge Discovery from Databases. Relations also occur in the areas of multi-level logic design and non-deterministic state machines. Hence Decomposition of Relations has applications in all these fields. Also with a Multi-Coloring we have the capability of creating relations even if the input data is a function. In the next section the Multi-Coloring algorithm will be presented and then it will be seen what kind of relations it can produce.

\subsection{A New Approach to Multi-Coloring using Dominations}

Here a new approach to Multi-Coloring is presented. The algorithm uses the same principle of dominations in an incompatibility graph, which was presented in Chapter 3. This algorithm has been given the name MISDOM which stands for Maximum Independent Sets using Dominations. In all latter sections, this algorithm will be referred to by this name. In the previous Chapter it was seen that an Exact Graph Coloring is not necessary to find the Column Multiplicity in Functional Decomposition, also from the results obtained in the previous Chapter, we saw that from a total number of program runs of $138, D O M$ gave the exact 


$$
\text { Sort_List }=\begin{array}{|l|l|l|l|l|l|l|l|}
\hline 7 & 5 & 4 & 2 & 1 & 6 & 3 & 0 \\
\hline
\end{array}
$$

(0)

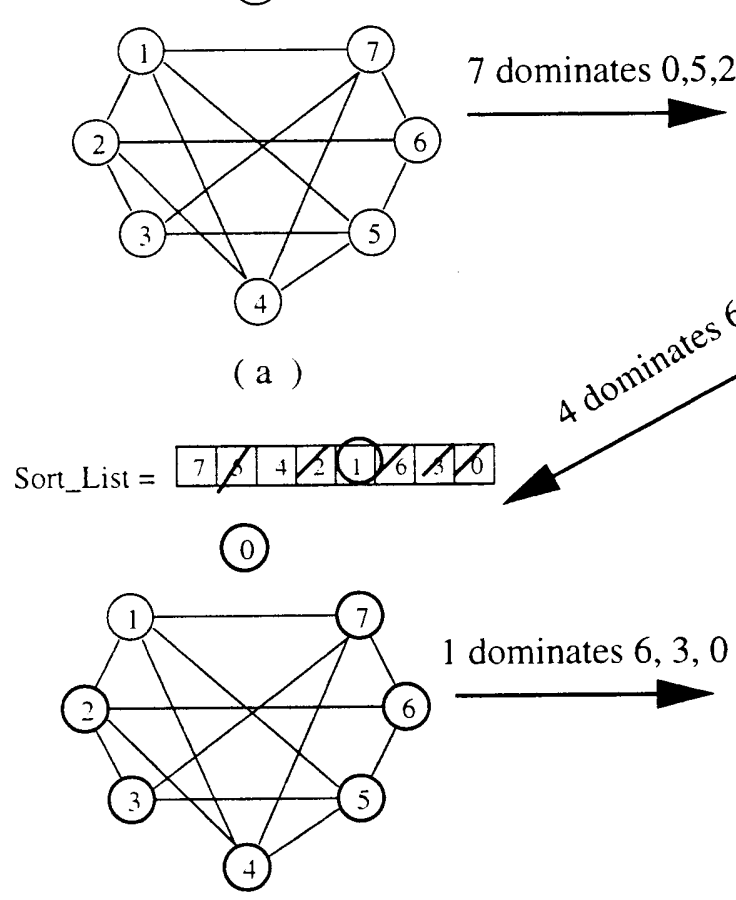

(c)
Sort_List $=$\begin{tabular}{|l|l|l|l|l|l|}
\hline $7 / 7(4)$ & 2 & 1 & 6 & 3 & 3 \\
\hline
\end{tabular}

(0)

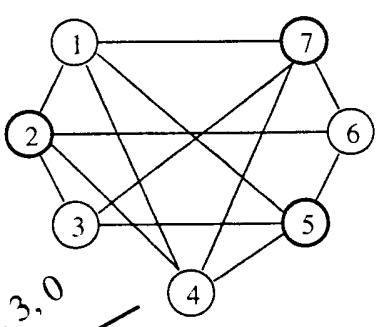

(b)

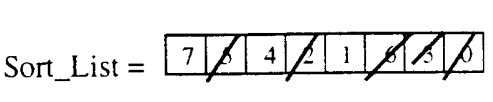

(0) $\mathrm{A}, \mathrm{B}, \mathrm{C}$

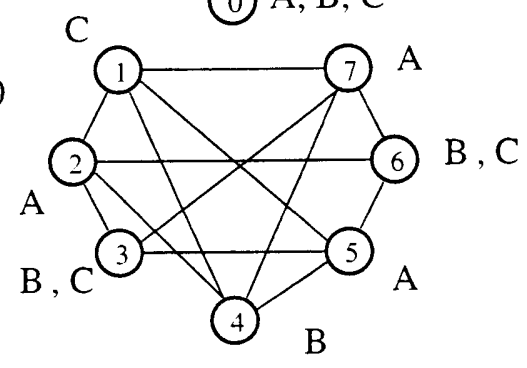

(d)

Figure 6.6: An Example showing how MISDOM colors a non cyclic Graph

solution in $95.6 \%$ of the cases, which proved that DOM is a very good heuristic. Hence we did not program a Multi-Coloring to be Exact. Instead it was decided to use an idea similar to $D O M$, thus ensuring that $M I S D O M$ will generate the same number of colors as $D O M$ on any incompatibility graph, the only difference being, $M I S D O M$ is a Multi-Coloring. To explain how MISDOM works, first an example is presented, which shows how MISDOM colors a graph. Then the Algorithm and Pseudo Code for MISDOM will be presented. 


\subsubsection{Example showing how MISDOM colors a non cyclic Graph}

Figure 6.6 shows an Example illustrating how the multi-coloring is done by MISDOM.

1. First the nodes of the incompatibility graph are sorted in a descending order of the vertex degrees, and a SORT_LIST is generated. The first element of SORT_LIST will have the node with the highest degree, and the last element of SORT_LIST will have the node with the smallest degree. The length of SORT_LIST will be equal to the number of nodes in the incompatibility graph. In Figure 6.6(a) the initial SORT_LIST and the initial Incompatibility Graph can be seen.

Select first node from SORT_LIST. Node 7 in this Example.

2. As can be seen from Figure 6.6(a) Node 7 dominates Node 0 and pseudo dominates Node 5 , and Node 2. This information is stored. Remove Node 0 , Node 5, and Node 2 from the SORT_LIST, this is shown in Figure 6.6(b). Now select the next node in SORT_LIST which is Node 4.

3. In Figure 6.6(c) it can be seen that Node 4 dominates Node 6, Node 3 and Node 0 . This information is stored, and Node 6, Node 3 and Node 0 are removed from the SORT_LIST if they have not already been removed. This is shown in Figure 6.6(c). Now take next node in SORT_LIST which is Node 1.

4. In the graph of Figure 6.6(d), Node 1 dominates Node 6, Node 3, and Node 0 . This information is stored, and Node 6, Node 3 and Node 0 are removed from the SORT_LIST if they have not already been removed. Now the end of SORT_LIST has been reached so the check for dominations terminates.

5. Now each Dominating Node is given a new color, and all the nodes it dominates are given the same color. If a node is dominated by more than one node it is given the colors of all the nodes that dominate it. 


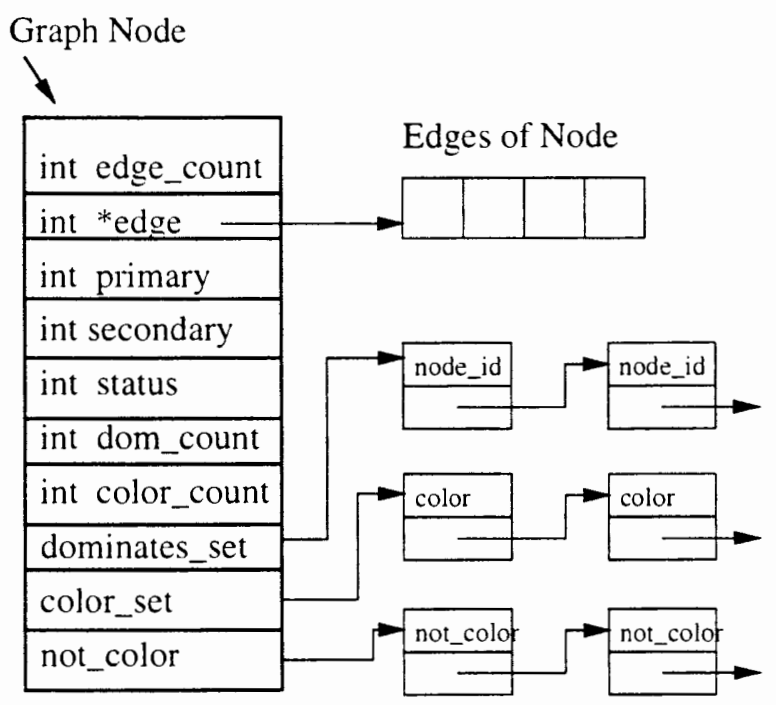

Figure 6.7: Data Structure used for a Node of the Incompatibility Graph

6. The final colored graph is shown in Figure 6.6(d). 3 colors were used to color the graph which is the minimum for this Graph. The color assignment is:

Color A $\{0,2,5,7\}$.

Color B $\{0,3,4,6\}$.

Color C $\{0,1,3,6\}$.

\subsubsection{Implementation Details for MISDOM}

Before going into the details of the Algorithm used by MISDOM a brief introduction to the data structures used by MISDOM is given below.

Figure 6.7 shows the data structure used to implement a Node in the incompatibility graph. In the graph node data structure shown in Figure 6.7, edge_count is the number of neighbors that the node has. edge is a pointer to the nodes that are the neighbors of the node. primary indicates that it is a primary node if set to "1". secondary indicates that it is a secondary node if set to "1". status is set to "1" if the node has been assigned a color, else it is set to " 0 ". dom_count is a count of the number of nodes that are dominated by 
this node. color_count is the number of colors that have been assigned to the node. dominates_set is a pointer to a link list which indicates which nodes are dominated by this node. color_set is a pointer to a link list containing all the colors that have been assigned to the node. not_color is a link list containing the colors that the node cannot be colored.

\subsubsection{Details of the Multi-Coloring Program, MISDOM}

The Algorithm for MISDOM has been divided into two parts:

1. Function check_dominations, which checks for dominations in the graph. It has two possible return values.

(i) Returns "TRUE" if graph was complete.

(ii) Returns "FALSE" if graph was cyclic, or disjoint.

2. Function graph_coloring is the top level function, which calls check_dominations.

The Algorithm for the Function graph_coloring has not been given here because it is exactly the same as the algorithm explained in Chapter 3 for DOM. The algorithm for the Function check_dominations is different and has been explained here.

\subsubsection{Algorithm for Function check_dominations}

NODES :is the set of nodes.

$N O D E_{-} C O U N T$ :is the number of nodes.

SORT_LIST :is an array of sorted nodes.

The first element in array SORT_LIST is the node with the most number of neighbors, and the last element is the node with the least number of neighbors. The length of SORT_LIST is equal to the number of nodes in the 
incompatibility graph.

1. Sort the nodes as explained in Step 1 of the example in Section 6.4.1 and generate a list SORT_LIST. Mark all nodes as being secondary initially ( set secondary in Figure 6.7 to "0").

2. Take one node from SORT_LIST, selected node, $S N=S O R T \_L I S T[\mathrm{i}]$. Mark this node as primary in the node data structure for this node.

3. Take one node from nodes ( NODE $=$ NODES[i]).

$/^{* *}$ Only check for dominations if $N O D E$ is a secondary node. All the information about the node is obtained from the data structure of the node shown in Figure 6.7. If a domination is found, the domination information is stored in the graph node of the dominating node. ${ }^{* *} /$

If ( NODE == secondary)

Check for Dominations of ( $S N, N O D E)$;

If Domination found, mark Dominated node as being dominated by $S N$. Do not remove Dominated node from graph, instead remove dominated node from SORT_LIST.

/* If node is a PRIMARY_NODE don't check it for Dominations */ else if ( NODE = primary )

go to Step 5

5. If the $S N$ node has not been checked with all the other nodes in NODES, go to Step 3, else go to Step 6.

6. If all the nodes in SORT_LIST have been checked go to Step 7, else go to Step 2.

7. Check if the nodes which are marked primary form a complete graph, if yes then return TRUE, else return FALSE. Here only the nodes which are marked primary need to be checked, because once a domination is found 
the dominated node is marked as secondary, and when a node has been marked secondary, it is never checked to see if this node dominates some other node, it is only checked to see if this node is dominated by some other node. But this is the same as removing a dominated node from the graph, hence only check the nodes which are marked as primary to see if they form a complete graph.

\subsubsection{Pseudo Code for Function check_dominations}

check_dominations()

\{

int nodejid, dominated;

int sort list; $\quad /^{*}$ Array containing the Sorted nodes */

NODE graph; $/ *$ Array of pointers to Graph Nodes */

/* A Graph Node was shown in Figure 6.7*/

/* Sort the nodes and save the order in array sort_list */

sort $\_$list $=$sort $\operatorname{nodes}()$;

$/ *$ Loop for the number of nodes */

for $(i=1 ; i \leq$ number_of_nodes; $i++)$

\{

${ }^{*}$ Take a node from the sort list */

node_id $=$ sort $\_$list $[i]$

$/ *$ If this node is a primary node do not continue */

if $($ graph[node_id] $\rightarrow$ primary $\neq 1$ )

\{

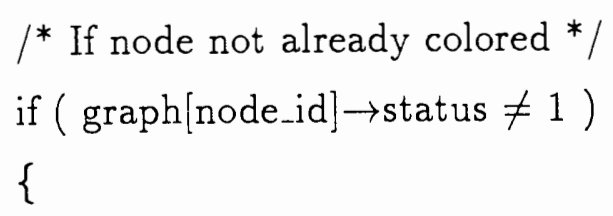

/ Since this node has been selected mark it as a primary node */ graph[node_id $] \rightarrow$ prim $=1$;

${ }^{*}$ Loop for this node with all the nodes */ 


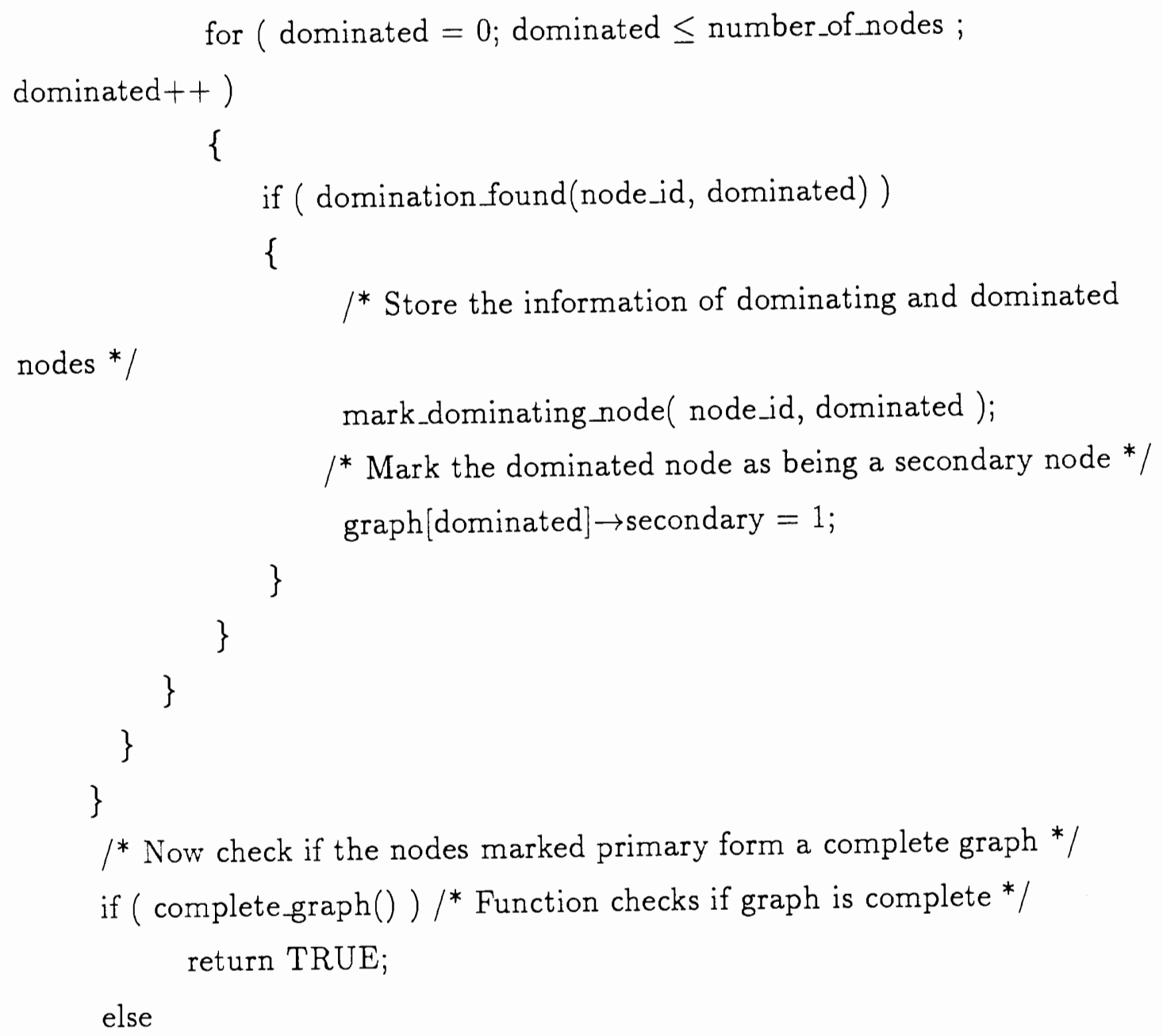

\subsubsection{Some Features of MISDOM}

MISDOM is a fast program which uses the principle of dominations to color a graph. Since this method uses the same principle of dominations explained earlier, MISDOM will not only give us a Multi-Coloring of a Graph, but will also find the exact minimum solution for all non-cyclic graphs. The algorithm for $M I S D O M$ is very similar to the algorithm for DOM presented earlier in Chapter 3. The way MISDOM handles cyclic graphs is exactly the same as the way DOM handles cyclic graphs. 
Theorem 6.3 If the incompatibility graph being colored is a non-cyclic graph then the independent sets produced by MISDOM will all be the Maximum Independent Sets. This means that each node in the incompatibility graph will be colored by the maximum number of colors that it can be colored with.

Proof 6.3 In order to check all the possible colors that a node can be colored with, the node must be checked with all the other nodes in the graph. But this is exactly what the function check_dominations does. When function check_dominations checks for dominations, it starts with the node with the most number of edges and then checks if it dominates any other nodes in the graph. Now this dominating node is not checked to see if any other node dominates it, but no node can possibly dominate this node because this node has the most number of edges. Also since the function check_dominations does not remove a dominated node from the graph once a domination is found, that dominated node is checked with all the other nodes too. Hence if the graph is a non-cyclic graph, then Theorem 6.3 will always be true.

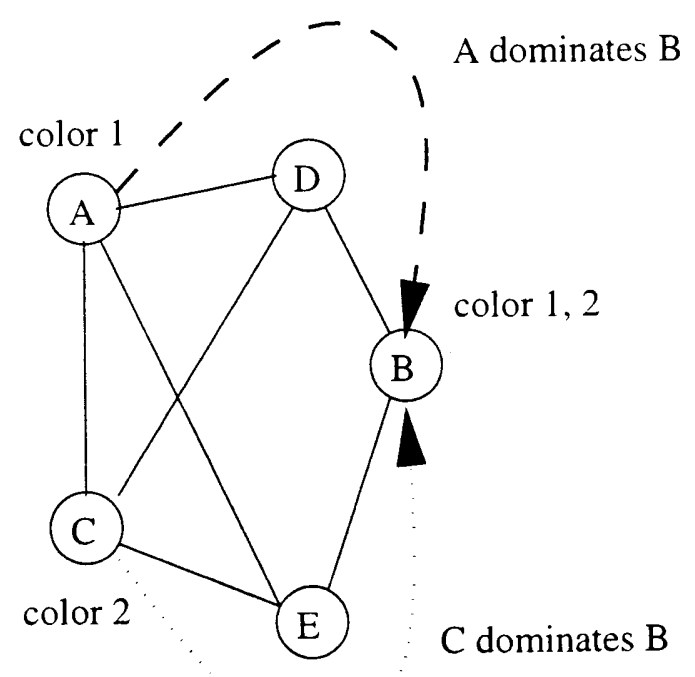

Figure 6.8: Reason why a dominated node can be colored all the colors of the nodes which dominate it 
Theorem 6.4 MISDOM will never produce Nodes with conflicting Colors when it colors a graph.

Proof 6.4 Consider the nodes " $A$ " and " $C$ " shown in Figure 6.8 which are incompatible with each other. As can be seen from Figure 6.8 node " $A$ " dominates node " $B$ ", which means that node " $B$ " does not have an edge with node " $A$ ". Since node " $C$ " also dominates node " $B$ " it means that node " $B$ " does not have an edge with node " $C$ ". Thus node " $B$ " can be colored the same colors as the colors assigned to nodes " $A$ " and node " $C$ ". Thus proving that there will never be a conflict.

The primary difference between $M I S D O M$ and DOM is that once a domination is found then the dominated node is not removed from the incompatibility graph. This is because only if it is found that these dominated nodes are dominated by some other node, can a Multi-Coloring of the incompatibility graph be found.

\subsection{Summary and Conclusions of Chapter 6}

In this Chapter the concept of Relations, and decomposition of relations was presented, and it was also shown how a Multi-Coloring can improve the quality of decompositions achieved. As the next step we actually have to test the MultiColoring in order to see if it really produces relations, and of what kind, in the decomposition of benchmark functions. This is presented in the next Chapter. 


\section{CHAPTER 7}

\section{AN EVALUATION OF THE MULTI-COLORING PROGRAM}

In this Chapter the testing of the Multi-Coloring is done in order to verify if the Multi-Coloring can produce relations from functions and thus improve the quality of the decompositions achieved This strategy has been taken among other reasons because we have no examples of real-life relations, and we did not want to test on randomly generated data only. In this Chapter first the Multi-Coloring is tested on random graphs and then the Multi-Coloring is tested on graphs generated from real life functions. Testing is done on both MCNC, and Machine Learning benchmarks. When testing the Multi-Coloring in decomposition it was realized that a new method of evaluating the cost function was required. Hence in this Chapter a new approach to calculate the cost function of a decomposed function or relation is presented.

\subsection{An Evaluation of Running MISDOM on randomly generated graphs}

In this section MISDOM is tested on randomly generated graphs. These graphs were generated with nodes from 10 to 100 and with edge percent from $10 \%$ to $90 \%$.

Table 7.1 shows how MISDOM performs on randomly generated graphs. In Table 7.1 the following notations are used:

$\mathrm{C}$ stands for the number of colors found.

NMOS stands for Number of nodes in more than one set. When MISDOM is used to calculate the column multiplicity in decomposition, NMOS will indicate the number of minterms in the $G$ block which correspond to generalized don't cares. 


\begin{tabular}{|c|c|c|c|c|c|c|c|c|c|c|c|c|}
\hline \multicolumn{13}{|c|}{ Nodes vs Edges } \\
\hline \multirow[t]{2}{*}{ Edge\% } & & \multicolumn{10}{|c|}{ Nodes } & \multirow[t]{2}{*}{ AVPCEF } \\
\hline & & 10 & 20 & 30 & 40 & 50 & 60 & 70 & 80 & 90 & 100 & \\
\hline \multirow[t]{3}{*}{$10 \%$} & $\mathrm{C}$ & 2 & 3 & 4 & 4 & 5 & 6 & 7 & 6 & 7 & 8 & \multirow{3}{*}{44.2} \\
\hline & NMOS & 5 & 11 & 15 & 14 & 20 & 32 & 31 & 29 & 40 & 46 & \\
\hline & $\mathrm{MCON}$ & 2 & 3 & 5 & 5 & 4 & 4 & 5 & 4 & 4 & 4 & \\
\hline \multirow[t]{3}{*}{$20 \%$} & $\mathrm{C}$ & 2 & 5 & 5 & 7 & 7 & 8 & 8 & 10 & 9 & 11 & \multirow{3}{*}{35.5} \\
\hline & NMOS & 0 & 9 & 11 & 20 & 17 & 21 & 27 & 31 & 24 & 35 & \\
\hline & $\mathrm{MCON}$ & 0 & 5 & 4 & 10 & 4 & 4 & 4 & 4 & 4 & 5 & \\
\hline \multirow[t]{3}{*}{$30 \%$} & $\bar{C}$ & 3 & 5 & 6 & 7 & 9 & 10 & 11 & 11 & 14 & 14 & \multirow{3}{*}{29.6} \\
\hline & NMOS & 3 & 9 & 7 & 11 & 17 & 22 & 21 & 14 & 30 & 29 & \\
\hline & $\mathrm{MCON}$ & 2 & 4 & 3 & 3 & 4 & 3 & 4 & 4 & 5 & 5 & \\
\hline \multirow[t]{3}{*}{$40 \%$} & $\bar{C}$ & 3 & 6 & 9 & 8 & 10 & 13 & 15 & 16 & 16 & 18 & \multirow{3}{*}{28.7} \\
\hline & NMOS & 1 & 7 & 12 & 8 & 12 & 19 & 22 & 27 & 22 & 28 & \\
\hline & MCON & 2 & 3 & 4 & 5 & 3 & 3 & 5 & 5 & 4 & 4 & \\
\hline \multirow[t]{3}{*}{$50 \%$} & $\mathrm{C}$ & 4 & 7 & 9 & 11 & 12 & 16 & 16 & 20 & 21 & 21 & \multirow{3}{*}{28.7} \\
\hline & NMOS & 3 & 7 & 8 & 10 & 13 & 19 & 18 & 25 & 29 & 26 & \\
\hline & $\mathrm{MCON}$ & 2 & 3 & 4 & 3 & 4 & 4 & 5 & 5 & 5 & 3 & \\
\hline \multirow[t]{3}{*}{$60 \%$} & $\mathrm{C}$ & 5 & 9 & 11 & 13 & 15 & 17 & 20 & 21 & 22 & 26 & \multirow{3}{*}{23.45} \\
\hline & NMOS & 2 & 6 & 8 & 8 & 13 & 14 & 15 & 21 & 16 & 26 & \\
\hline & MCON & 3 & 4 & 3 & 3 & 3 & 3 & 4 & 3 & 3 & 4 & \\
\hline \multirow[t]{3}{*}{$70 \%$} & $\mathrm{C}$ & 6 & 9 & 13 & 16 & 19 & 18 & 23 & 28 & 28 & 31 & \multirow{3}{*}{22} \\
\hline & NMOS & 1 & 6 & 6 & 9 & 12 & 10 & 19 & 20 & 18 & 20 & \\
\hline & $\mathrm{MCON}$ & 3 & 2 & 3 & 5 & 4 & 3 & 3 & 4 & 4 & 4 & \\
\hline \multirow[t]{3}{*}{$80 \%$} & $\mathrm{C}$ & 6 & 9 & 15 & 17 & 21 & 24 & 25 & 31 & 34 & 37 & \multirow{3}{*}{16.7} \\
\hline & NMOS & 1 & 4 & 7 & 5 & 8 & 12 & 8 & 16 & 15 & 16 & \\
\hline & $\mathrm{MCON}$ & 2 & 2 & 3 & 3 & 4 & 3 & 4 & 4 & 4 & 4 & \\
\hline \multirow[t]{3}{*}{$90 \%$} & $\mathrm{C}$ & 7 & 11 & 16 & 21 & 27 & 31 & 32 & 38 & 41 & 48 & \multirow{3}{*}{13} \\
\hline & NMOS & 0 & 1 & 2 & 5 & 9 & 12 & 7 & 12 & 9 & 17 & \\
\hline & MCON & 0 & 2 & 2 & 2 & 3 & 3 & 2 & 3 & 4 & 4 & \\
\hline
\end{tabular}

Table 7.1: An Evaluation of running MISDOM on Randomly generated graphs

MCON stands for the maximum colors assigned to one node. When MISDOM is used to calculate the column multiplicity in Functional Decomposition, MCON will indicate the input cube or input minterm which has the largest number of values in the output.

Two trends can be observed from this Table. On moving along a row of the Table 7.1, that is for increasing nodes for a constant edge percent, NMOS increases, 
and on moving along a column of the Table 7.1, that is for increasing edge per-

cent for a constant number of nodes, NMOS decreases. In the last column of Table 7.1 AVPCEP stands for the average percent for constant edge percent. This is obtained by totaling all the NMOS in one row and dividing it by the total of the number of nodes. Hence this indicates what is the average percent of NMOS for a given percent of edges in the graph. From this Table it can be seen that MISDOM generates Maximum Independent sets (explained in Chapter 6 Section 6.1.2) with a large number of overlapping minterms. This is very desirable from our perspective because we want to create more generalized don't cares in the $\mathrm{G}$ functions. How generalized don't cares can improve the quality of achieved decompositions was introduced in Chapter 6.

As the next step it was required to test $M I S D O M$ on graphs generated during the process of Functional Decomposition to see if it can generate large overlaps in the Maximum Independent Sets in the graphs that are generated during Functional Decomposition, and thus produce a higher number of generalized don't cares.

\subsection{A New Approach to calculate the cost function of a Decomposed Function or Relation}

In Chapter $5 D F C$ was introduced for binary functions and for multi-valued functions. The $D F C$ cost function has been proved to be a good evaluation of the cost function for binary functions in Machine Learning, but $D F C$ does not provide a good evaluation for multi-valued functions and for relations. Also $D F C$ does not take into account the circuit complexity of the decomposed function. By circuit complexity we mean how easy or how difficult it would be to realize this circuit in terms of gates. It was decided therefore to develop a new cost function which would not only consider the generalized don't cares while evaluating the cost, but would also take into account the circuit complexity of the decomposed function. Hence there are two factors being considered in the cost function: 


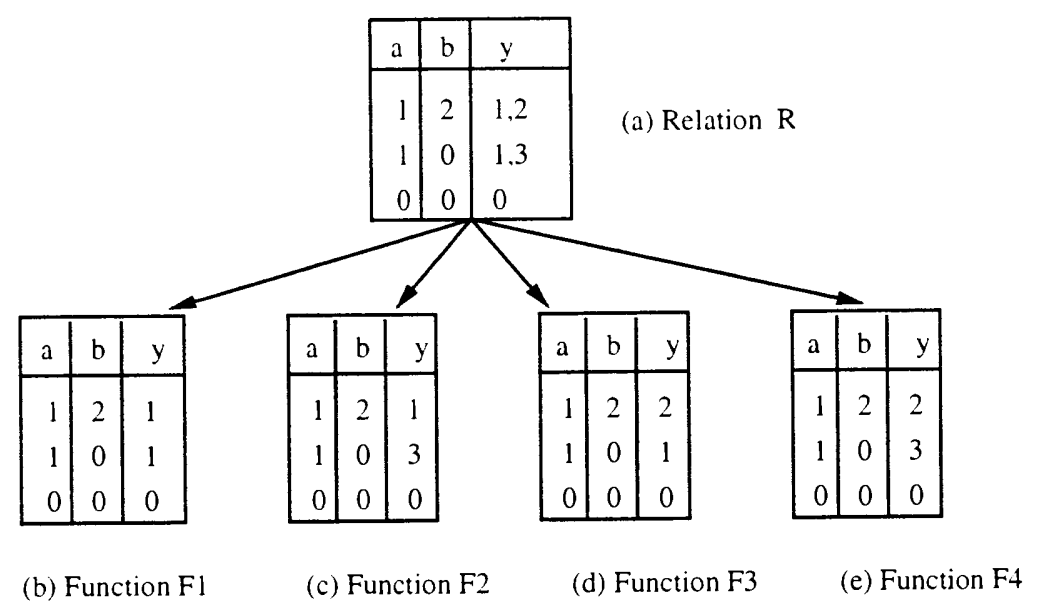

Figure 7.1: Different classes of Functions possible from a Relation

1. The first factor calculates the total number of functions obtained by selecting different don't cares of the relation. This cost function is called NOFP, which stands for number of functions possible.

2. The second factor taken into account is the simplicity of the Karnaugh map of the function or relation. This cost function has been given the name COSC, which stands for cost of simplest circuit.

The Cost Functions NOFP, and COSC are explained in the next two Sections with the help of examples.

\subsubsection{Calculating NOFP for a Relation and a Function}

NOFP takes into account the number of combinations obtained by the introduction of generalized don't cares in a function. If, for example, a generalized don't care in a relation has two possible values, it means that there are now two possible functions, one for each of the values of the generalized don't cares. Hence NOFP calculates the number of all possible combinations of classes of functions that can be obtained from a relation, by making choices in the generalized don't cares in the relation. Each class of function obtained from a 


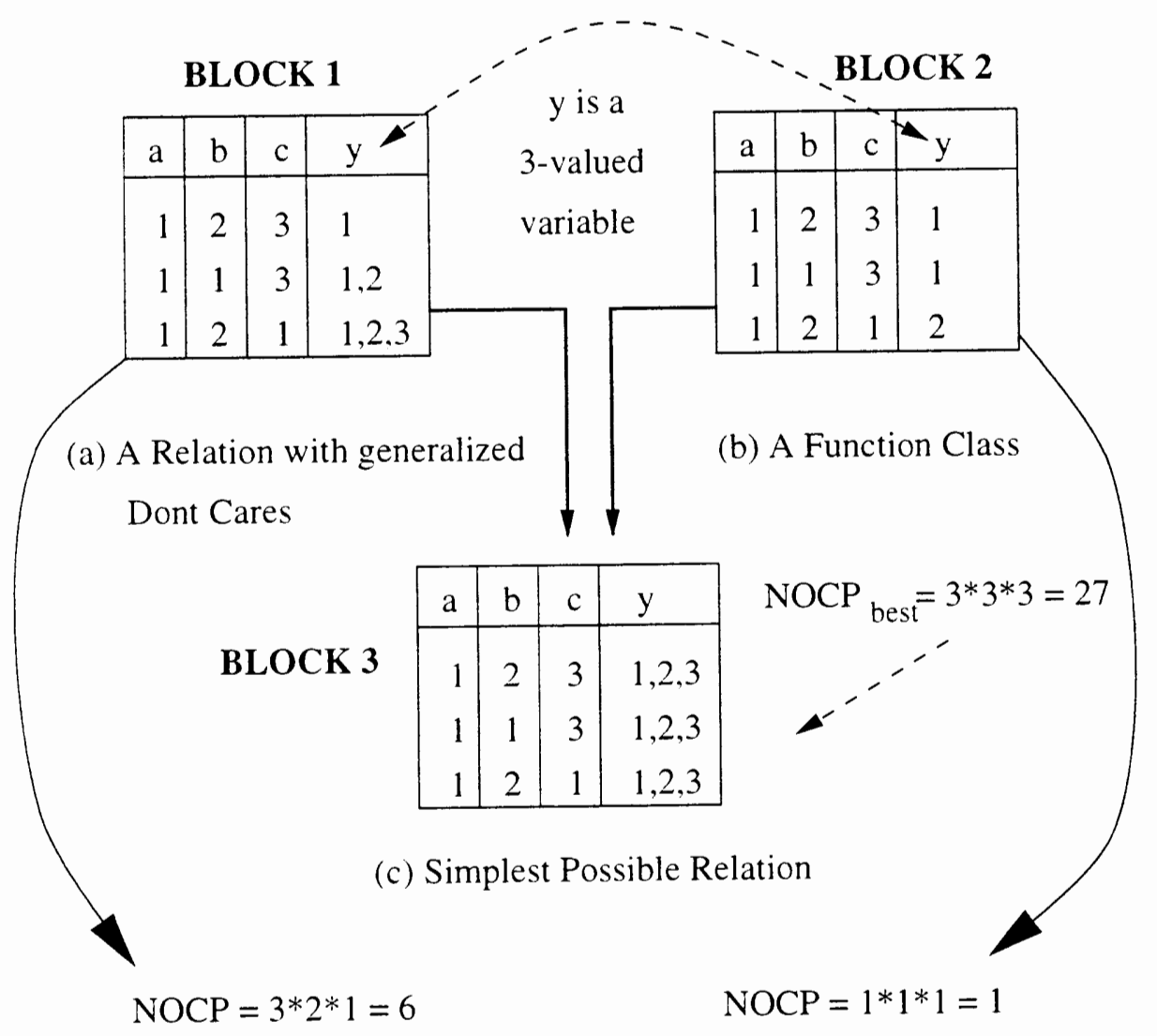

Figure 7.2: A Comparison of the calculation of NOFP for a Relation and for a Function 
Relation will have the same inputs but different outputs. Figure 7.1(a) shows a relation with two inputs and one output. Figure 7.1(b), 7.1(c), 7.1(d) and 7.1(e) show the four possible functions obtained from the relation in Figure 7.1(a). As can be seen each of these functions is a subset of the original relation. Figure 7.2 shows how NOFP is calculated. Figure 7.2(a) shows a relation having three threevalued inputs, and one three-valued output. Figure 7.2(b) shows a function which has three three-valued inputs and one three-valued output. As can be seen, the function and the relation both have the same inputs, the function has only one possible output, while the relation has generalized don't cares in the output.

Definition 7.1 For a block with number of multi-values in the output $=\mathbf{n m v}$, and number of rows in the block = rows, the maximum number of combinations of maps possible for this block (NOF $\left.P_{\text {best }}\right)$ is defined as NOF $P_{\text {best }}=(n m v)^{\text {rows }}$

The block labeled BLOCK 3 in Figure 7.2(c) shows NOFP $P_{\text {best }}$ for the blocks labeled BLOCK 1 and BLOCK 2 in Figure 7.2(a) and Figure 7.2(b), respectively. Now for BLOCK 1 the number of possible combinations is 6 , hence NOFP for the relation shown in Figure 7.2(a) is 6, and NOFP for the function shown in Figure 7.2(b) is 1 . For a function NOFP will always be equal to one. The higher the value of NOFP, the better the block.

\subsubsection{Calculating $C O S C$ for a Relation and a Function}

COSC calculates what is the cost of implementing a decomposed function or relation. COSC considers two factors in finding out the cost of the function or relation:

The first factor considered is the number of overlaps in the output, where two minterms overlap if they have a common output. But this does not really illustrate how simple it would be to realize the function or relation. Figure 7.3(a) shows a function with 8 minterms having the same output one, hence it has 8 overlaps, and Figure 7.3(b) shows a function having 4 minterms having the same output one, hence it has 4 overlaps. Now, as can be seen from Figure 7.3, even 


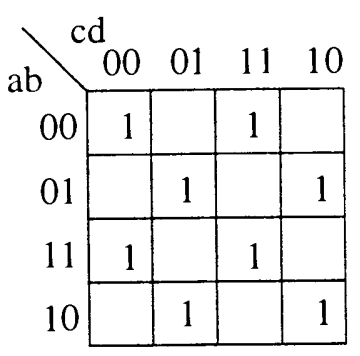

(a) Function with 8 repetitions.

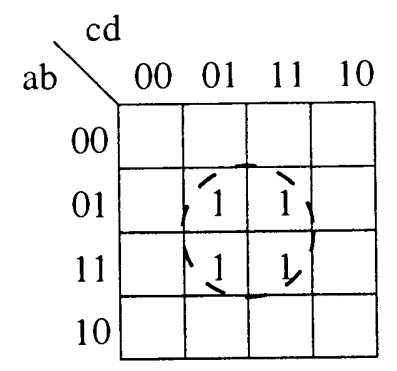

(b) Function with 4 repetitions

Figure 7.3: A Comparison showing that more repetions of common variables in not necessarily better

though the function in Figure 7.3(a) has more overlaps than the function in Figure 7.3(b), the function in Figure 7.3(b) would be simpler to realize in terms of gates than the function shown in Figure 7.3(a).

In Figure 7.3 it was shown that more overlaps is not necessarily a good thing. What is needed, is to check if for every set of two cubes that overlap the Hamming distance of these two cubes is equal to one? This is the second factor considered while calculating COSC. Figure 7.4 shows how COSC can be calculated for a relation. Figure 7.4(a) shows a Table of a relation $F$ having three inputs and one output. Figure 7.4(b) shows the Karnaugh map of $F$. Now in order to calculate $C O S C$ for $F$, a compatibility graph is created. In the compatibility graph each node represents a row of the Table of the relation $F$. In this compatibility graph any two nodes $N 1$ and $N 2$ have an edge between them if nodes $N 1$ and $N 2$ have a Hamming distance of one, and if they have a common output. Thus since minterm "1101" and minterm "1111" have a Hamming distance of one, and since they both have a common output which is a "2", they have an edge. The edges weight shown in the compatibility graph correspond to the output(or outputs) common to the nodes having this edge. In order to calculate $C O S C$, each minterm in the relation is checked with all the other minterms in the relation and if they can have an edge then the cost COSC is incremented by two(because two minterms 


\begin{tabular}{|c|c|c|c|c|}
\hline $\mathrm{a}$ & $\mathrm{b}$ & $\mathrm{c}$ & $\mathrm{d}$ & $\mathrm{y}$ \\
\hline 1 & 1 & 0 & 1 & 1,2 \\
1 & 1 & 1 & 1 & 2 \\
1 & 0 & 0 & 1 & 1,3 \\
0 & 1 & 0 & 1 & $1,2,3$ \\
1 & 0 & 0 & 0 & 2,3 \\
1 & 0 & 1 & - & 3 \\
0 & 0 & 1 & 1 & 2 \\
0 & 0 & 0 & - & 0 \\
0 & 0 & 1 & 0 & 0 \\
0 & 1 & 0 & 0 & 0 \\
0 & 1 & 1 & 1 & 0 \\
1 & 1 & 0 & 0 & 0 \\
1 & 1 & 1 & 0 & 0 \\
\hline
\end{tabular}

\begin{tabular}{|c|c|c|c|c|}
\hline$a b$ & 00 & 01 & 11 & 10 \\
\hline 00 & 0 & 0 & 2 & 0 \\
\hline 01 & 0 & $1,2,3$ & 0 & $1,2,3$ \\
\hline 11 & 0 & 1,2 & 2 & 0 \\
\hline & 2.3 & $\overline{1}, 3$ & -3 & $-\overline{3}$, \\
\hline
\end{tabular}

(b) Karnaugh Map of Relation F

(a) Table of Relation $\mathrm{F}$

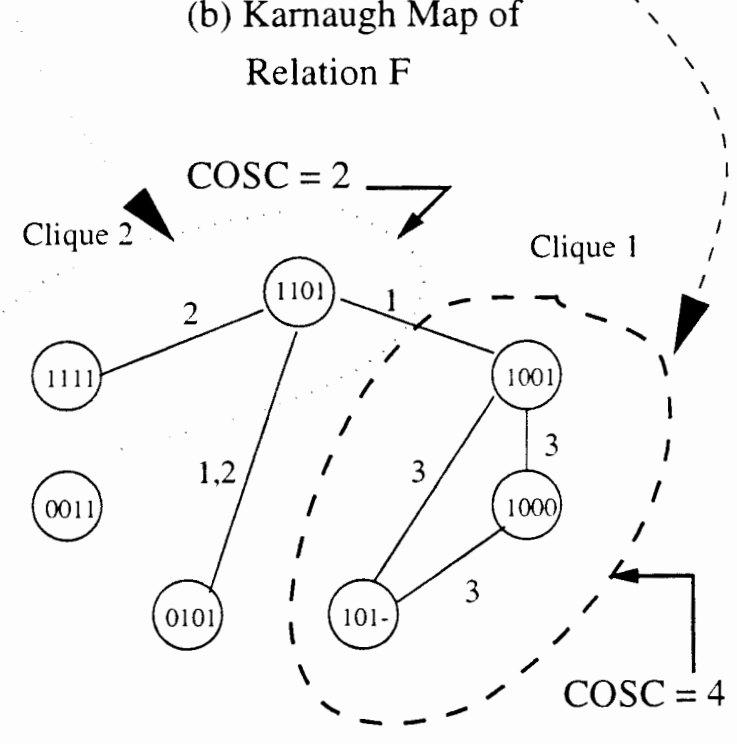

(c) Compatibility graph of Relation $\mathrm{F}$

Figure 7.4: Example showing how $C O S C$ is calculated 
are being considered). Thus COSC reflects the number of minterms which can be combined with other minterms. For Clique 1 shown in Figure 7.4(c) the cost is two since two minterms are being combined. For Clique 2 shown in Figure 7.4(c) the cost is four since there are four possible combinations of the minterms. In the calculation of COSC all the cliques are considered while calculating the cost, including the overlapping cliques.

\subsection{An Evaluation of Running MISDOM on Graphs generated during Decomposition of Functions and Relations}

In this section a comparison is done to see if $M I S D O M$ can improve the quality of decompositions achieved. The cost factors NOFP and COSC will be used to evaluate the quality of the decomposed functions. A comparison is done on functions from Circuit, and Machine Learning Benchmarks. In order to do the comparison it was needed to compare a Graph Coloring and a Multi-Coloring. For this experiment $D O M$ was chosen as the Graph Coloring program, and the Multi-Coloring program is MISDOM. The results of the testing has been divided into two parts, first the testing is done on the Circuit benchmarks, and then the testing is done on the Machine Learning Benchmarks. MVGUD was tested with two, four, and five variables in the Bound set. The following points help to understand the values of NOFP and COSC in all the Tables shown.

1. While analyzing these Tables, it should be remembered, that the higher the values of $N O F P$ and $C O S C$, the better is the decomposition.

2. The values shown for NOFP and COSC in the Tables are the sums of the values of NOFP and COSC for each block in the decomposed functions or relations.

3. If the value of $N O F P$ is equal to one, it means that there were no generalized don't cares in the output for the decomposed function or relation. When 


\begin{tabular}{|l|c|c|c|c|c|c|c|}
\hline \multicolumn{4}{|c|}{ Function } & \multicolumn{4}{|c|}{ Algorithm } \\
\hline & & & & \multicolumn{2}{|c|}{ DOM } & \multicolumn{2}{c|}{ MISDOM } \\
\hline name & in & out & cubes & NOFP & COSC & NOFP & COSC \\
\hline 5xp1 & 7 & 10 & 143 & 1 & 250 & 2 & 224 \\
\hline 9sym1 & 9 & 1 & 158 & 1 & 62 & 1 & 1372 \\
\hline b12 & 15 & 9 & 172 & 1 & 280 & 1 & 248 \\
\hline con1 & 7 & 2 & 18 & 1 & 96 & 1 & 70 \\
\hline bw & 5 & 28 & 97 & 1 & 618 & 25 & 718 \\
\hline ex5p & 8 & 63 & 214 & 1 & 2488 & 2 & 3272 \\
\hline misex1 & 8 & 7 & 40 & 1 & 214 & 1 & 334 \\
\hline rd53 & 5 & 3 & 63 & 1 & 46 & 1 & 48 \\
\hline rd73 & 7 & 3 & 274 & 1 & 52 & 1 & 78 \\
\hline rd84 & 8 & 4 & 515 & 1 & 106 & 1 & 170 \\
\hline sao2 & 10 & 4 & 133 & 1 & 512 & 1 & 526 \\
\hline squar5 & 5 & 8 & 56 & 1 & 166 & 1 & 172 \\
\hline xor5 & 5 & 1 & 32 & 1 & 1 & 1 & 1 \\
\hline
\end{tabular}

Table 7.2: $M V G U D$ run with 2 variables in the bound set on MCNC Benchmarks

$D O M$ is used in $M V G U D$ to find the column multiplicity, $N O F P$ will always be equal to one.

4. If the value of $C O S C$ is equal to one it means that there were no cubes or minterms in the decomposed blocks of the function that could be combined. Thus if the value of $C O S C$ is one for a function it implies that this function would be very costly in terms of gates to implement.

\subsubsection{A Comparison of MISDOM and DOM on MCNC Benchmarks}

Table 7.2 shows the results of running $M V G U D$ with two variables in the bound set, Table 7.3 shows the results of running MVGUD with four variables in the bound set, and Table 7.4 shows the results of running MVGUD with five variables in the bound set. Looking at the values of NOFP in Table 7.2 it can be seen that MISDOM finds a NOFP better than one for only three benchmarks. For a total number of program runs of fourteen, DOM has a better $C O S C$ in three cases, while MISDOM is better in eleven cases. DOM and MISDOM will generate 


\begin{tabular}{|l|c|c|c|c|c|c|c|}
\hline \multicolumn{4}{|c|}{ Function } & \multicolumn{4}{c|}{ Algorithm } \\
\hline & & & & \multicolumn{3}{|c|}{ DOM } & MISDOM \\
\hline name & in & out & cubes & NOFP & COSC & NOFP & COSC \\
\hline 5xp1 & 7 & 10 & 143 & 1 & 914 & 1 & 532 \\
\hline 9sym1 & 9 & 1 & 158 & 1 & 188 & 1 & 572 \\
\hline b12 & 15 & 9 & 172 & 1 & 784 & 1 & 658 \\
\hline con1 & 7 & 2 & 18 & 1 & 80 & 1 & 80 \\
\hline bw & 5 & 28 & 97 & 1 & 1162 & 14 & 1314 \\
\hline ex5p & 8 & 63 & 214 & 1 & 7228 & 1 & 8782 \\
\hline misex1 & 8 & 7 & 40 & 1 & 794 & 1 & 940 \\
\hline rd53 & 5 & 3 & 63 & 1 & 60 & 1 & 70 \\
\hline rd73 & 7 & 3 & 274 & 1 & 616 & 1 & 596 \\
\hline rd84 & 8 & 4 & 515 & 1 & 214 & 1 & 360 \\
\hline sao2 & 10 & 4 & 133 & 1 & 4726 & 1 & 2364 \\
\hline squar5 & 5 & 8 & 56 & 1 & 354 & 1 & 360 \\
\hline xor5 & 5 & 1 & 32 & 1 & 1 & 1 & 1 \\
\hline
\end{tabular}

Table 7.3: $M V G U D$ run with 4 variables in the bound set on MCNC Benchmarks

\begin{tabular}{|l|c|c|c|c|c|c|c|}
\hline \multicolumn{4}{|c|}{ Function } & \multicolumn{4}{c|}{ Algorithm } \\
\hline & & & & \multicolumn{2}{c|}{ DOM } & \multicolumn{2}{c|}{ MISDOM } \\
\hline name & in & out & cubes & NOFP & COSC & NOFP & COSC \\
\hline 5xp1 & 7 & 10 & 143 & 1 & 822 & 1 & 804 \\
\hline 9sym1 & 9 & 1 & 158 & 1 & 1842 & 1 & 2422 \\
\hline b12 & 15 & 9 & 172 & 1 & 1574 & 1 & 1502 \\
\hline con1 & 7 & 2 & 18 & 1 & 200 & 1 & 200 \\
\hline bw & 5 & 28 & 97 & 1 & 2236 & 1 & 2236 \\
\hline ex5p & 8 & 63 & 214 & 1 & 19832 & 1 & 21010 \\
\hline misex1 & 8 & 7 & 40 & 1 & 896 & 1 & 864 \\
\hline rd53 & 5 & 3 & 63 & 1 & 1850 & 1 & 1850 \\
\hline rd73 & 7 & 3 & 274 & 1 & 190 & 1 & 228 \\
\hline rd84 & 8 & 4 & 515 & 1 & 928 & 1 & 1120 \\
\hline sao2 & 10 & 4 & 133 & 1 & 3016 & 1 & 3248 \\
\hline squar5 & 5 & 8 & 56 & 1 & 782 & 1 & 782 \\
\hline xor5 & 5 & 1 & 32 & 1 & 1 & 1 & 1 \\
\hline
\end{tabular}

Table 7.4: MVGUD run with 5 variables in the bound set on MCNC Benchmarks 
the same number of colors for the graphs, but due to the different strategies employed in the two programs, for the same incompatibility graph $D O M$ might give the nodes different colors than MISDOM, so the columns of the Karnaugh map of the function, corresponding to the nodes of the incompatibility graph will have different codes assigned to them. Hence due to this the Karnaugh map of the decomposed function can be different for when DOM is used or when MISDOM is used. Thus it is possible for DOM to have a better COSC than MISDOM, but in most cases $C O S C$ for $M I S D O M$ will be better.

Looking at the values of NOFP for MISDOM in Tables 7.3 and 7.3 , it can be seen that MISDOM fails to introduce generalized don't cares in most cases. The reason why $M I S D O M$ fails to introduce generalized don't cares in most cases for $M C N C$ benchmarks is because these benchmarks have very few don't cares, and the graphs generated during decomposition for these benchmarks are dense graphs, hence in most cases the nodes have only one possible color.

\subsubsection{Summary of the results of testing MISDOM on MCNC bench- marks}

The primary advantage, that we want to gain by using a Multi-Coloring in Decomposition is the introduction of generalized don't cares. Hence from the results of testing on the $M C N C$ benchmarks it can be concluded that the Multi-Coloring will not improve the quality of decompositions achieved on circuit benchmarks. Now it was required to see if the results differ on Machine Learning Benchmarks. The results of testing on Machine Learning Benchmarks is presented in the next section. 


\subsubsection{A Comparison of MISDOM and DOM on Machine Learning Benchmarks}

Two experiments were performed here: In Experiment 1 the number of variables in the Bound Set were increased and DOM and MISDOM were compared, in this experiment the number of don't cares in the Machine Learning Functions were kept constant. In Experiment 2 the number of don't cares in the functions were increased and then DOM and MISDOM were compared by running MVGUD with different variables in the Bound Set.

\subsubsection{Experiment 1: Running $M V G U D$ on 8 variable Machine Learn- ing Benchmarks with $70 \%$ don't cares}

In this Experiment MVGUD was run with MISDOM and DOM on the 8 variable Machine Learning Benchmarks, and the number of don't cares in these Benchmarks was $70 \%$ and the number of variables in the Bound Set was increased. Tables 7.5, 7.6, 7.7, and 7.8 show the results of running MVGUD with two, four, five, and six variables in the Bound Set respectively. Here a big difference is observed when compared to the results obtained on MCNC benchmarks. As can be seen from these Tables, MISDOM is able to find overlaps in the graphs generated during decomposition and is thus able to introduce generalized don't cares in the decomposed functions. Also it is observed from these Tables that as the size of the Bound Set increases the number of generalized don't cares introduced also increases. Also from these Tables it is observed that whenever NOFP is high, the COSC for the benchmark is also very good, which is obvious because now there are more possible combinations, and thus the decomposed functions can be much simpler. 


\begin{tabular}{|c|c|c|c|c|c|c|c|}
\hline \multicolumn{8}{|c|}{ Comparison of Multi-Coloring and Coloring on Machine Learning Benchmark } \\
\hline \multicolumn{4}{|c|}{ Function } & \multicolumn{4}{|c|}{ Algorithm } \\
\hline & & & & & & & $\overline{O M}$ \\
\hline name & in & out & cubes & NOFP & $\operatorname{COSC}$ & NOFP & $\operatorname{COSC}$ \\
\hline addo & 8 & 1 & 77 & 1 & 36 & 1 & 46 \\
\hline add2 & 8 & 1 & 77 & 1 & 28 & 2 & 42 \\
\hline add4 & 8 & 1 & 77 & 1 & 4 & 1 & 4 \\
\hline and_or_chain8 & 8 & 1 & 77 & 1 & 20 & 1 & 28 \\
\hline $\operatorname{ch} 15 f 0$ & 8 & 1 & 77 & 1 & 52 & 32 & 100 \\
\hline $\operatorname{ch} 176 \mathrm{fo}$ & 8 & 1 & 77 & 1 & 16 & 1 & 16 \\
\hline ch177fo & 8 & 1 & 77 & 1 & 42 & 1 & 40 \\
\hline $\operatorname{ch} 22 \mathrm{fo}$ & 8 & 1 & 77 & 1 & $\overline{44}$ & 10 & 74 \\
\hline $\operatorname{ch} 30 \mathrm{fO}$ & 8 & 1 & 77 & 1 & 38 & 4 & 50 \\
\hline $\operatorname{ch} 47 \mathrm{fO}$ & 8 & 1 & 77 & 1 & 48 & 219 & 78 \\
\hline $\operatorname{ch} 52 \mathrm{f} 4$ & 8 & 1 & 77 & 1 & 24 & 2 & 34 \\
\hline $\operatorname{ch} 70 \mathrm{f3}$ & 8 & 1 & 77 & 1 & 34 & 4 & 74 \\
\hline $\operatorname{ch} 74 \mathrm{f1}$ & 8 & 1 & 77 & 1 & $\overline{48}$ & 22 & 98 \\
\hline $\operatorname{ch} 83 \mathrm{f} 2$ & 8 & 1 & 77 & 1 & 20 & 4 & 32 \\
\hline ch8fo & 8 & 1 & 77 & 1 & 44 & 10 & 82 \\
\hline contains_4_ones & 8 & 1 & 77 & 1 & 36 & 6 & 40 \\
\hline greater_than & 8 & 1 & 77 & 1 & 76 & $\overline{19}$ & 226 \\
\hline interval1 & 8 & 1 & 77 & 1 & 60 & 10 & 80 \\
\hline interval2 & 8 & 1 & 77 & 1 & 12 & 1 & 28 \\
\hline $\mathrm{kddl}$ & 8 & 1 & 77 & 1 & 20 & 1 & 28 \\
\hline kdd2 & 8 & 1 & 77 & 1 & 12 & 1 & 12 \\
\hline kdd3 & 8 & 1 & 77 & 1 & 1 & 1 & 1 \\
\hline kdd4 & 8 & 1 & 77 & 1 & 34 & 2 & 64 \\
\hline kdd5 & 8 & 1 & 77 & 1 & 12 & 1 & 12 \\
\hline kdd6 & 8 & 1 & 77 & 1 & 44 & 2 & 88 \\
\hline $\mathrm{kdd} 7$ & 8 & 1 & 77 & 1 & 8 & 1 & 8 \\
\hline kdd8 & 8 & 1 & 77 & 1 & 20 & 1 & 40 \\
\hline kdd9 & 8 & 1 & 77 & 1 & 38 & 1 & 38 \\
\hline majority gate & 8 & 1 & 77 & 1 & 48 & 6 & 60 \\
\hline modulus2 & 8 & 1 & 77 & 1 & 44 & 6 & 56 \\
\hline monkish1 & 8 & 1 & 77 & 1 & 8 & 1 & 8 \\
\hline monkish2 & 8 & 1 & 77 & 1 & 18 & 1 & 18 \\
\hline monkish3 & 8 & 1 & 77 & 1 & 22 & 2 & 48 \\
\hline $\operatorname{mux} 8$ & 8 & 1 & 77 & 1 & 30 & 2 & 40 \\
\hline nnr1 & 8 & 1 & 77 & 1 & 46 & 8 & 68 \\
\hline nกr2 & 8 & 1 & 77 & 1 & 14 & 1 & 14 \\
\hline$n n r^{3}$ & 8 & 1 & 77 & 1 & 68 & 276 & 110 \\
\hline or_and_chain 8 & 8 & 1 & 77 & 1 & 20 & 1 & 30 \\
\hline pal & 8 & 1 & 77 & 1 & 28 & 1 & 46 \\
\hline pal_dbl_output & 8 & 1 & 77 & 1 & 62 & 78 & 138 \\
\hline parity & 8 & 1 & 77 & 1 & 1 & 1 & 1 \\
\hline primes 8 & 8 & 1 & 77 & 1 & 1 & 1 & 1 \\
\hline remainder 2 & 8 & 1 & 77 & 1 & 86 & 10 & 78 \\
\hline rnd 1 & 8 & 1 & 77 & 1 & 100 & 4 & 126 \\
\hline rnd2 & 8 & 1 & 77 & 1 & 52 & 160 & 160 \\
\hline rnd3 & 8 & 1 & 77 & 1 & 56 & 48 & 128 \\
\hline
\end{tabular}

Table 7.5: $M V G U D$ run with 2 variables in the bound set on 8 variable Machine Learning Benchmarks with $70 \%$ don't cares 


\begin{tabular}{|c|c|c|c|c|c|c|c|}
\hline \multicolumn{8}{|c|}{ Comparison of Multi-Coloring and Coloring on Machine Learning Benchmarks } \\
\hline \multicolumn{4}{|c|}{ Function } & \multicolumn{4}{|c|}{ Algorithm } \\
\hline & & & & \multicolumn{2}{|c|}{$D O M$} & \multicolumn{2}{|c|}{$M I S D O M$} \\
\hline name & in & $\overline{\text { out }}$ & cubes & NOFP & $\overline{C O S C}$ & NOFP & $\operatorname{COSC}$ \\
\hline addo & 8 & 1 & 77 & 1 & 1 & 4 & 102 \\
\hline add2 & 8 & 1 & 77 & 1 & 82 & 280 & 140 \\
\hline add 4 & 8 & 1 & 77 & 1 & 32 & 1 & 32 \\
\hline and_or_chain8 & 8 & 1 & 77 & 1 & 64 & 1 & 108 \\
\hline ch15fo & 8 & 1 & 77 & 1 & $\overline{66}$ & 80 & 138 \\
\hline $\operatorname{ch} 176 \mathrm{fO}$ & 8 & 1 & 77 & 1 & 84 & 1 & 88 \\
\hline $\operatorname{ch} 177 \mathrm{fO}$ & 8 & 1 & 77 & 1 & 32 & 1 & 106 \\
\hline $\operatorname{ch} 22 \mathrm{fo}$ & 8 & 1 & 77 & 1 & 82 & 1 & 82 \\
\hline $\operatorname{ch} 30 \mathrm{fo}$ & 8 & 1 & 77 & 1 & 76 & 24 & 134 \\
\hline $\operatorname{ch} 47 \mathrm{fO}$ & 8 & 1 & 77 & 1 & 72 & 66 & 112 \\
\hline $\operatorname{ch} 52 f 4$ & 8 & 1 & 77 & 1 & 66 & 8 & 112 \\
\hline $\operatorname{ch} 70 \mathrm{f} 3$ & 8 & 1 & 77 & 1 & 102 & 64 & 126 \\
\hline $\operatorname{ch} 83 \mathrm{f} 2$ & 8 & 1 & 77 & 1 & 60 & 40 & 96 \\
\hline $\operatorname{ch} 8 \mathrm{fO}$ & 8 & 1 & 77 & 1 & 88 & 1 & 100 \\
\hline contains_4_ones & 8 & 1 & 77 & 1 & 40 & 34 & 70 \\
\hline greater_than & 8 & 1 & 77 & 1 & 72 & 258 & 136 \\
\hline interval1 & 8 & 1 & 77 & 1 & 80 & 20 & 120 \\
\hline interval2 & 8 & 1 & 77 & 1 & 92 & 16 & 332 \\
\hline kdd1 & 8 & 1 & 77 & 1 & 18 & 1 & 18 \\
\hline kdd2 & 8 & 1 & 77 & 1 & 96 & 1 & 96 \\
\hline kdd3 & 8 & 1 & 77 & 1 & 78 & 1 & 100 \\
\hline kdd4 & 8 & 1 & 77 & 1 & 20 & 1 & 20 \\
\hline kdd5 & 8 & 1 & 77 & 1 & 88 & 128 & 146 \\
\hline kdd6 & 8 & 1 & 77 & 1 & 56 & 1 & 56 \\
\hline kdd7 & 8 & 1 & 77 & 1 & 80 & 1 & 86 \\
\hline kdd8 & 8 & 1 & 77 & 1 & 16 & 1 & 16 \\
\hline kdd9 & 8 & 1 & 77 & 1 & 76 & 1 & 94 \\
\hline majority gate & 8 & 1 & 77 & 1 & 62 & 68 & 116 \\
\hline modulus2 & 8 & 1 & 77 & 1 & 68 & 34 & 134 \\
\hline monkish1 & 8 & 1 & 77 & 1 & 32 & 1 & 32 \\
\hline monkish2 & 8 & 1 & 77 & 1 & 46 & 8 & $\overline{72}$ \\
\hline monkish3 & 8 & 1 & 77 & 1 & 80 & $\overline{1}$ & 106 \\
\hline $\operatorname{mux} 8$ & 8 & 1 & 77 & 1 & 92 & 136 & 148 \\
\hline nnrl & 8 & 1 & 77 & 1 & 76 & 1 & 76 \\
\hline nnr2 & 8 & 1 & 77 & 1 & 40 & 1 & 40 \\
\hline nnr3 & 8 & 1 & 77 & 1 & $\overline{96}$ & 332 & 160 \\
\hline or_and_chain8 & 8 & 1 & 77 & 1 & 104 & 1 & 104 \\
\hline pal & 8 & 1 & 77 & 1 & 80 & 1 & 102 \\
\hline pal_dbl_output & 8 & 1 & 77 & 1 & 80 & 4114 & 162 \\
\hline parity & 8 & 1 & 77 & 1 & 1 & 1 & 1 \\
\hline primes 8 & 8 & 1 & 77 & 1 & 1 & 1 & 1 \\
\hline remainder2 & 8 & 1 & 77 & 1 & 60 & 304 & 140 \\
\hline rnd1 & 8 & 1 & $\overline{77}$ & 1 & 66 & 2064 & 160 \\
\hline rnd2 & 8 & 1 & $\overline{77}$ & 1 & 80 & 8204 & 146 \\
\hline rnd3 & 8 & 1 & 77 & 1 & 92 & 262176 & 210 \\
\hline
\end{tabular}

Table 7.6: $M V G U D$ run with 4 variables in the bound set on 8 variable Machine Learning Benchmarks with $70 \%$ don't cares 


\begin{tabular}{|c|c|c|c|c|c|c|c|}
\hline \multicolumn{8}{|c|}{ Comparison of Multi-Coloring and Coloring on Machine Learning Benchmarks } \\
\hline & & & & & & $M I S D$ & \\
\hline name & in & out & cubes & $\overline{N O F P}$ & $\overline{C O S C}$ & NOFP & $\operatorname{COSC}$ \\
\hline addo & 8 & 1 & 77 & 1 & 110 & $\overline{64}$ & 176 \\
\hline add2 & 8 & 1 & 77 & 1 & 88 & 1 & 88 \\
\hline add4 & 8 & 1 & 77 & 1 & 132 & 1 & 132 \\
\hline and_or_chain8 & 8 & 1 & 77 & 1 & 140 & 1 & 258 \\
\hline $\operatorname{ch} 15 \mathrm{fO}$ & 8 & 1 & 77 & 1 & 126 & 13824 & 352 \\
\hline $\operatorname{ch} 176 \mathrm{fo}$ & 8 & 1 & 77 & 1 & 128 & 1 & 210 \\
\hline ch177fo & 8 & 1 & 77 & 1 & 126 & 1 & 138 \\
\hline $\operatorname{ch} 22 \mathrm{fO}$ & 8 & 1 & 77 & 1 & 134 & 1 & 150 \\
\hline ch30fo & 8 & 1 & 77 & 1 & 128 & 1024 & 278 \\
\hline ch47fo & 8 & 1 & 77 & 1 & 128 & 128 & 316 \\
\hline ch52f4 & 8 & 1 & 77 & 1 & 136 & 18432 & 610 \\
\hline ch70f3 & 8 & 1 & 77 & 1 & 112 & 8192 & 454 \\
\hline $\operatorname{ch} 74 \mathrm{f} 1$ & 8 & 1 & 77 & 1 & 112 & 373248 & 436 \\
\hline $\operatorname{ch} 83 \mathrm{f} 2$ & 8 & 1 & 77 & 1 & 118 & 32768 & 316 \\
\hline ch8fo & 8 & 1 & 77 & 1 & 72 & 256 & 210 \\
\hline contains_4_ones & 8 & 1 & 77 & 1 & 92 & 1 & 192 \\
\hline greater_than & 8 & 1 & 77 & 1 & 116 & 1024 & 246 \\
\hline interval 1 & 8 & 1 & 77 & 1 & 142 & 62208 & 390 \\
\hline interval2 & 8 & 1 & 77 & 1 & 14 & 1 & 14 \\
\hline kdd 1 & 8 & 1 & 77 & 1 & 142 & 1 & 204 \\
\hline kdd2 & 8 & 1 & 77 & 1 & 146 & 1 & 146 \\
\hline kdd3 & 8 & 1 & 77 & 1 & 16 & 1 & 16 \\
\hline kdd 4 & 8 & 1 & 77 & 1 & 142 & 16 & 332 \\
\hline kdd5 & 8 & 1 & 77 & 1 & 122 & 1 & 198 \\
\hline $\mathrm{kdd} 6$ & 8 & 1 & 77 & 1 & 142 & 16 & 212 \\
\hline kdd7 & 8 & 1 & 77 & 1 & 128 & 1 & 224 \\
\hline kdd8 & 8 & 1 & 77 & 1 & 134 & 64 & 304 \\
\hline kdd9 & 8 & 1 & 77 & 1 & 152 & 8192 & 326 \\
\hline majority gate & 8 & 1 & 77 & 1 & $\overline{94}$ & 256 & $\overline{342}$ \\
\hline modulus2 & 8 & 1 & 77 & 1 & 124 & 512 & 392 \\
\hline monkish1 & 8 & 1 & 77 & 1 & 132 & 1 & 132 \\
\hline monkish2 & 8 & 1 & 77 & 1 & 94 & 4096 & 236 \\
\hline monkish3 & 8 & 1 & 77 & 1 & 110 & 1 & 222 \\
\hline mux8 & 8 & 1 & 77 & 1 & 144 & 64 & 230 \\
\hline nnr1 & 8 & 1 & 77 & 1 & 132 & 64 & 238 \\
\hline nnr2 & 8 & 1 & 77 & 1 & 150 & 1 & 150 \\
\hline nnr3 & 8 & 1 & 77 & 1 & 156 & 1492992 & 644 \\
\hline or_and_chain 8 & 8 & 1 & 77 & 1 & 134 & 1 & 232 \\
\hline pal & 8 & 1 & 77 & 1 & 112 & 1 & 250 \\
\hline pal_dbl_output & 8 & 1 & 77 & 1 & 118 & 124416 & 380 \\
\hline parity & 8 & 1 & 77 & 1 & 1 & 1 & 1 \\
\hline primes 8 & 8 & 1 & 77 & 1 & 1 & 1 & 1 \\
\hline remainder 2 & 8 & 1 & 77 & 1 & 98 & 746496 & 318 \\
\hline rnd1 & 8 & 1 & 77 & 1 & 130 & 995328 & 460 \\
\hline rnd2 & 8 & 1 & 77 & 1 & 124 & 106168320 & 452 \\
\hline $\operatorname{rnd} 3$ & 8 & 1 & $\overline{77}$ & 1 & 146 & 165888 & 426 \\
\hline
\end{tabular}

Table 7.7: MVGUD run with 5 variables in the bound set on 8 variable Machine Learning Benchmarks with $70 \%$ don't cares 


\begin{tabular}{|l|c|c|c|c|c|c|c|}
\hline \multicolumn{2}{|c|}{ Comparison of Multi-Coloring and Coloring on Machine Learning Benchmarks } \\
\hline \multicolumn{2}{|c|}{ Function } & \multicolumn{4}{c|}{ Algorith m } \\
\hline & in & out & cubes & NOFP & COSC & NOFP & COSC \\
\hline name & 8 & 1 & 77 & 1 & 174 & 1 & 174 \\
\hline add0 & 8 & 1 & 77 & 1 & 80 & 1 & 80 \\
\hline add2 & 8 & 1 & 77 & 1 & 168 & 1 & 168 \\
\hline add4 & 8 & 1 & 77 & 1 & 202 & 1 & 202 \\
\hline and_or_chain8 & 8 & 1 & 77 & 1 & 126 & 1 & 126 \\
\hline ch15f0 & 8 & 1 & 77 & 1 & 162 & 2097152 & 232 \\
\hline ch176fo & 8 & 1 & 77 & 1 & 162 & 1 & 162 \\
\hline ch177f0 & 8 & 1 & 77 & 1 & 118 & 67108864 & 240 \\
\hline ch22f0 & 8 & 1 & 77 & 1 & 120 & 32768 & 208 \\
\hline ch30f0 & 8 & 1 & 77 & 1 & 76 & 18874368 & 250 \\
\hline ch47f0 & 8 & 1 & 77 & 1 & 94 & 1729626112 & 322 \\
\hline ch52f4 & 8 & 1 & 77 & 1 & 144 & 256 & 218 \\
\hline ch74f1 & 8 & 1 & 77 & 1 & 104 & 2031390720 & 290 \\
\hline ch83f2 & 8 & 1 & 77 & 1 & 164 & 131072 & 206 \\
\hline ch8f0 & 8 & 1 & 77 & 1 & 60 & 131072 & 136 \\
\hline contains_4_ones & 8 & & & & & & 1 \\
\hline greater_than & 8 & 1 & 77 & 1 & 144 & 8192 & 186 \\
\hline interval1 & 8 & 1 & 77 & 1 & 96 & 1820327936 & 282 \\
\hline interval2 & 8 & 1 & 77 & 1 & 124 & 1586446336 & 286 \\
\hline kdd1 & 8 & 1 & 77 & 1 & 170 & 1 & 170 \\
\hline kdd2 & 8 & 1 & 77 & 1 & 220 & 1 & 220 \\
\hline kdd3 & 8 & 1 & 77 & 1 & 164 & 1 & 164 \\
\hline kdd4 & 8 & 1 & 77 & 1 & 4 & 1 & 4 \\
\hline kdd5 & 8 & 1 & 77 & 1 & 186 & 1 & 186 \\
\hline kdd6 & 8 & 1 & 77 & 1 & 204 & 1 & 204 \\
\hline kdd7 & 8 & 1 & 77 & 1 & 146 & 32 & 164 \\
\hline kdd8 & 8 & 1 & 77 & 1 & 156 & 524288 & 228 \\
\hline kdd9 & 8 & 1 & 77 & 1 & 98 & 63700992 & 236 \\
\hline kdd10 & 8 & 1 & 77 & 1 & 132 & 1 & 132 \\
\hline majority_gate & 8 & 1 & 77 & 1 & 108 & 1787822080 & 266 \\
\hline modulus2 & 8 & 1 & 77 & 1 & 112 & 1146617856 & 280 \\
\hline monkish1 & 8 & 1 & 77 & 1 & 172 & 1 & 172 \\
\hline monkish2 & 8 & 1 & 77 & 1 & 94 & 1820327936 & 280 \\
\hline monkish3 & 8 & 1 & 77 & 1 & 188 & 1 & 188 \\
\hline
\end{tabular}

Table 7.8: MVGUD run with 6 variables in the bound set on 8 variable Machine Learning Benchmarks with $70 \%$ don't cares 
7.3.2.2 Experiment 2: Running $M V G U D$ on 8 variable Machine Learning Benchmarks with $90 \%$ don't cares

In this Experiment, the don't cares in the eight variable Machine Learning Benchmarks were increased to $90 \%$ and MVGUD was tested on these Benchmarks with two, four, five, and six variables in the Bound set.

Tables 7.9, 7.10, 7.11, and 7.12 show the results for testing MVGUD with two, four, five, and six variables in the Bound set respectively. On comparing these Tables with the Tables from Experiment 1 it is seen that as the number of don't cares increases, NOFP also increases. 


\begin{tabular}{|c|c|c|c|c|c|c|c|}
\hline \multicolumn{8}{|c|}{ Comparison of Multi-Coloring and Coloring on Machine Learning Benchmark } \\
\hline & & & & & & & $\overline{O M}$ \\
\hline name & in & out & cubes & NOFP & $\operatorname{COSC}$ & NOFP & $\operatorname{COSC}$ \\
\hline addo & 8 & 1 & 77 & 1 & 24 & 2 & 26 \\
\hline add2 & 8 & 1 & 77 & 1 & 18 & 5 & 28 \\
\hline add4 & 8 & 1 & 77 & 1 & 1 & 1 & 1 \\
\hline and_or_chain8 & 8 & 1 & 77 & 1 & 12 & 1 & 12 \\
\hline ch15fo & 8 & 1 & 77 & 1 & 44 & 14 & 82 \\
\hline $\mathrm{ch} 176 \mathrm{fo}$ & 8 & 1 & 77 & 1 & 16 & 1 & 16 \\
\hline $\operatorname{ch} 177 \mathrm{fO}$ & 8 & 1 & 77 & 1 & 1 & 1 & 1 \\
\hline $\operatorname{ch} 22 \mathrm{fO}$ & 8 & 1 & 77 & 1 & 14 & 1 & 14 \\
\hline $\operatorname{ch} 30 \mathrm{fo}$ & 8 & 1 & 77 & 1 & 12 & 1 & 12 \\
\hline ch47fo & 8 & 1 & 77 & 1 & 18 & 4 & 26 \\
\hline $\operatorname{ch} 52 \mathrm{f} 4$ & 8 & 1 & 77 & 1 & 28 & 4 & 42 \\
\hline $\operatorname{ch} 74 \mathrm{f} 1$ & 8 & 1 & 77 & 1 & 16 & 2 & 18 \\
\hline $\operatorname{ch} 83 \mathrm{f} 2$ & 8 & 1 & 77 & 1 & 26 & 10 & 66 \\
\hline ch8fo & 8 & 1 & 77 & 1 & 1 & 1 & 1 \\
\hline contains_4_ones & 8 & 1 & 77 & 1 & 14 & 6 & 44 \\
\hline greater_than & 8 & 1 & 77 & 1 & 20 & 1 & 20 \\
\hline interval1 & 8 & 1 & 77 & 1 & 30 & 10 & 108 \\
\hline interval2 & 8 & 1 & 77 & 1 & 28 & 30 & 96 \\
\hline$\overline{k d d 1}$ & 8 & 1 & 77 & 1 & 16 & 4 & 32 \\
\hline kdd2 & 8 & 1 & 77 & 1 & 12 & 2 & 20 \\
\hline kdd3 & 8 & 1 & 77 & 1 & 16 & 1 & 16 \\
\hline kdd4 & 8 & 1 & 77 & 1 & 4 & 1 & 4 \\
\hline kdd5 & 8 & 1 & 77 & 1 & 20 & 1 & 20 \\
\hline kdd6 & 8 & 1 & 77 & 1 & 12 & 2 & 20 \\
\hline kdd7 & 8 & 1 & 77 & 1 & 16 & 2 & 24 \\
\hline kdd8 & 8 & 1 & 77 & 1 & 18 & 1 & 18 \\
\hline kdd9 & 8 & 1 & 77 & 1 & 14 & 4 & 22 \\
\hline kdd10 & 8 & 1 & 77 & 1 & 30 & 2 & 24 \\
\hline majority gate & 8 & 1 & 77 & 1 & 28 & 8 & 58 \\
\hline modulus2 & 8 & 1 & 77 & 1 & 18 & 1 & 18 \\
\hline monkish 1 & 8 & 1 & 77 & 1 & 1 & 1 & 1 \\
\hline monkish2 & 8 & 1 & 77 & 1 & 20 & 2 & 38 \\
\hline monkish3 & 8 & 1 & 77 & 1 & 18 & 2 & 22 \\
\hline
\end{tabular}

Table 7.9: $M V G U D$ run with 2 variables in the bound set on 8 variable Machine Learning Benchmarks with $90 \%$ don't cares 


\begin{tabular}{|c|c|c|c|c|c|c|c|}
\hline \multicolumn{8}{|c|}{$\begin{array}{c}\text { Comparison of Multi-Coloring and Coloring on Machine Learning Benchmark } \\
\text { Function }\end{array}$} \\
\hline & \multicolumn{2}{|c|}{$D O M$} & \multicolumn{2}{|c|}{$\overline{M I S D O M}$} \\
\hline name & in & out & cubes & NOFP & $\operatorname{COSC}$ & NOFP & COSC \\
\hline addo & 8 & 1 & 77 & 1 & 44 & 24 & $6 \overline{2}$ \\
\hline add2 & 8 & 1 & 77 & 1 & 26 & 6 & 56 \\
\hline$\overline{\mathrm{add} 4}$ & 8 & 1 & $7 \overline{7}$ & 1 & 46 & 8 & 56 \\
\hline and_or_chain8 & 8 & 1 & 77 & 1 & 34 & $\overline{1}$ & 34 \\
\hline $\operatorname{ch} 15 \mathrm{fo}$ & 8 & 1 & 77 & 1 & 42 & 72 & 76 \\
\hline ch176fo & 8 & 1 & 77 & 1 & 36 & 8 & 62 \\
\hline $\operatorname{chi77f0}$ & 8 & 1 & 77 & 1 & 60 & 48 & 82 \\
\hline $\operatorname{ch} 22 \mathrm{fo}$ & 8 & 1 & 77 & 1 & 28 & 1 & 28 \\
\hline $\operatorname{ch} 30 \mathrm{fo}$ & 8 & 1 & 77 & 1 & 58 & 132 & 90 \\
\hline $\operatorname{ch} 47 f 0$ & 8 & 1 & 77 & 1 & 38 & 32 & 66 \\
\hline $\operatorname{ch} 52 \mathrm{f} 4$ & 8 & 1 & 77 & 1 & 46 & 24 & 82 \\
\hline $\operatorname{ch} 74 f 1$ & 8 & 1 & 77 & 1 & 48 & 256 & 80 \\
\hline $\operatorname{ch} 83 \mathrm{f} 2$ & 8 & 1 & 77 & 1 & 54 & 36 & 92 \\
\hline$\overline{\operatorname{ch} 8 f 0}$ & 8 & 1 & 77 & 1 & 1 & 1 & 1 \\
\hline contains_4_ones & 8 & 1 & 77 & 1 & 28 & 136 & 82 \\
\hline greater_than & 8 & 1 & 77 & 1 & 64 & 1 & 64 \\
\hline interval1 & 8 & 1 & 77 & 1 & 28 & 40 & 68 \\
\hline interval2 & 8 & 1 & 77 & 1 & 36 & 48 & 76 \\
\hline kdd1 & 8 & 1 & 77 & 1 & 26 & 1 & 26 \\
\hline kdd2 & 8 & 1 & 77 & 1 & 20 & 1 & 20 \\
\hline kdd3 & 8 & 1 & 77 & 1 & 56 & 8 & 76 \\
\hline kdd4 & 8 & 1 & 77 & 1 & 1 & 1 & 1 \\
\hline kdd5 & 8 & 1 & 77 & 1 & 56 & 34 & 82 \\
\hline$\overline{\mathrm{kdd} 6}$ & 8 & 1 & 77 & 1 & 42 & 1 & 42 \\
\hline kdd? & 8 & 1 & 77 & 1 & 30 & 34 & 56 \\
\hline kdd8 & 8 & 1 & 77 & 1 & 10 & 1 & 10 \\
\hline kdd9 & 8 & 1 & 77 & 1 & 40 & 24 & 72 \\
\hline kdd 10 & 8 & 1 & 77 & 1 & 44 & 40 & 92 \\
\hline majority gate & 8 & 1 & 77 & 1 & 38 & 24 & 70 \\
\hline modulus2 & 8 & 1 & 77 & 1 & $\overline{52}$ & 18 & 70 \\
\hline monkish I & 8 & 1 & 77 & 1 & 1 & 1 & 1 \\
\hline monkish2 & 8 & 1 & 77 & 1 & 38 & 36 & 70 \\
\hline monkish3 & 8 & 1 & 77 & 1 & 54 & 72 & 98 \\
\hline
\end{tabular}

Table 7.10: $M V G U D$ run with 4 variables in the bound set on 8 variable Machine Learning Benchmarks with $90 \%$ don't cares 


\begin{tabular}{|l|c|c|c|c|c|c|c|}
\hline \multicolumn{2}{|c|}{ Comparison of Multi-Coloring and Coloring on Machine Learning Benchmarks } \\
\hline \multicolumn{2}{|c|}{ Function } & \multicolumn{4}{c|}{ Algorith m } \\
\hline name & in & out & cubes & NOFP & COSC & NOFP & COSC \\
\hline add0 & 8 & 1 & 77 & 1 & 56 & 64 & 100 \\
\hline add2 & 8 & 1 & 77 & 1 & 46 & 2 & 52 \\
\hline add4 & 8 & 1 & 77 & 1 & 50 & 4 & 62 \\
\hline and_or_chain8 & 8 & 1 & 77 & 1 & 36 & 8 & 82 \\
\hline ch15f0 & 8 & 1 & 77 & 1 & 32 & 2 & 44 \\
\hline ch176f0 & 8 & 1 & 77 & 1 & 42 & 1 & 42 \\
\hline ch177f0 & 8 & 1 & 77 & 1 & 56 & 16 & 70 \\
\hline ch22f0 & 8 & 1 & 77 & 1 & 12 & 1 & 12 \\
\hline ch30f0 & 8 & 1 & 77 & 1 & 46 & 64 & 90 \\
\hline ch47f0 & 8 & 1 & 77 & 1 & 34 & 8 & 72 \\
\hline ch52f4 & 8 & 1 & 77 & 1 & 64 & 64 & 90 \\
\hline ch74f1 & 8 & 1 & 77 & 1 & 56 & 4096 & 70 \\
\hline ch83f2 & 8 & 1 & 77 & 1 & 52 & 32 & 80 \\
\hline ch8f0 & 8 & 1 & 77 & 1 & 1 & 1 & 1 \\
\hline contains_4_ones & 8 & 1 & 77 & 1 & 42 & 4 & 54 \\
\hline greater_than & 8 & 1 & 77 & 1 & 54 & 1 & 64 \\
\hline interval1 & 8 & 1 & 77 & 1 & 44 & 256 & 98 \\
\hline interval2 & 8 & 1 & 77 & 1 & 36 & 4 & 72 \\
\hline kdd1 & 8 & 1 & 77 & 1 & 14 & 1 & 14 \\
\hline kdd2 & 8 & 1 & 77 & 1 & 18 & 1 & 18 \\
\hline kdd3 & 8 & 1 & 77 & 1 & 52 & 16 & 78 \\
\hline kdd4 & 8 & 1 & 77 & 1 & 16 & 1 & 16 \\
\hline kdd5 & 8 & 1 & 77 & 1 & 52 & 2 & 76 \\
\hline kdd6 & 8 & 1 & 77 & 1 & 56 & 2048 & 94 \\
\hline kdd7 & 8 & 1 & 77 & 1 & 42 & 8 & 66 \\
\hline kdd8 & 8 & 1 & 77 & 1 & 52 & 4 & 82 \\
\hline kdd9 & 8 & 1 & 77 & 1 & 40 & 16 & 70 \\
\hline kdd10 & 8 & 1 & 77 & 1 & 56 & 4 & 68 \\
\hline majority_gate & 8 & 1 & 77 & 1 & 38 & 4 & 76 \\
\hline modulus2 & 8 & 1 & 77 & 1 & 42 & 8 & 62 \\
\hline monkish1 & 8 & 1 & 77 & 1 & 1 & 1 & 1 \\
\hline monkish2 & 8 & 1 & 77 & 1 & 60 & 512 & 88 \\
\hline monkish3 & 8 & 1 & 77 & 1 & 58 & 4096 & 110 \\
\hline
\end{tabular}

Table 7.11: $M V G U D$ run with 5 variables in the bound set on 8 variable Machine Learning Benchmarks with $90 \%$ don't cares 


\begin{tabular}{|c|c|c|c|c|c|c|c|}
\hline \multicolumn{8}{|c|}{ Comparison of Multi-Coloring and Coloring on Machine Learning Benchmarks } \\
\hline & & & & & & & $\overline{O M}$ \\
\hline name & in & out & cubes & NOFP & $\overline{C O S C}$ & NOFP & $\operatorname{COSC}$ \\
\hline addo & 8 & 1 & 77 & 1 & 56 & 512 & 60 \\
\hline add2 & 8 & 1 & 77 & 1 & 42 & 1 & 42 \\
\hline $\operatorname{add} 4$ & 8 & 1 & 77 & 1 & 50 & 1 & 50 \\
\hline and_or_chain8 & 8 & 1 & 77 & 1 & 44 & 1024 & 70 \\
\hline ch15fo & 8 & 1 & 77 & 1 & 30 & 4 & 34 \\
\hline $\operatorname{ch} 176 \mathrm{fo}$ & 8 & 1 & 77 & 1 & 44 & 1 & 44 \\
\hline $\operatorname{ch} 177 \mathrm{fO}$ & 8 & 1 & 77 & 1 & 42 & 1 & 42 \\
\hline ch22fo & 8 & 1 & 77 & 1 & 38 & 64 & 60 \\
\hline $\operatorname{ch} 30 f 0$ & 8 & 1 & 77 & 1 & 52 & 64 & 54 \\
\hline ch47fo & 8 & 1 & 77 & 1 & 26 & 32 & 44 \\
\hline $\operatorname{ch} 52 f 4$ & 8 & 1 & 77 & 1 & 48 & 256 & 62 \\
\hline $\operatorname{ch} 74 \mathrm{f} 1$ & 8 & 1 & 77 & 1 & 50 & 256 & 74 \\
\hline ch83f2 & 8 & 1 & 77 & 1 & 42 & 1 & 40 \\
\hline ch8fo & 8 & 1 & 77 & 1 & 1 & 1 & 1 \\
\hline contains_4_ones & 8 & 1 & 77 & 1 & 28 & 8 & 38 \\
\hline greater_than & 8 & 1 & 77 & 1 & 56 & 1 & 56 \\
\hline intervall & 8 & 1 & 77 & 1 & 24 & 8 & 32 \\
\hline interval2 & 8 & 1 & 77 & 1 & 46 & 16 & 60 \\
\hline kdd1 & 8 & 1 & 77 & 1 & 30 & 256 & 54 \\
\hline kdd2 & 8 & 1 & 77 & 1 & 44 & 2048 & 56 \\
\hline kdd3 & 8 & 1 & 77 & 1 & 36 & 16 & 52 \\
\hline kdd4 & 8 & 1 & 77 & 1 & 4 & 1 & 4 \\
\hline kdd5 & 8 & 1 & 77 & 1 & 40 & 8 & 68 \\
\hline kdd6 & 8 & 1 & 77 & 1 & 36 & 16384 & 64 \\
\hline kdd7 & 8 & 1 & 77 & 1 & 64 & 1 & 64 \\
\hline kdd8 & 8 & 1 & 77 & 1 & 50 & 8 & 64 \\
\hline kdd9 & 8 & 1 & 77 & 1 & 32 & 62208 & 90 \\
\hline kdd10 & 8 & 1 & 77 & 1 & 42 & 1 & 42 \\
\hline majority gate & 8 & 1 & 77 & 1 & 32 & 128 & 66 \\
\hline modulus2 & 8 & 1 & 77 & 1 & 30 & 8 & 46 \\
\hline monkish 1 & 8 & 1 & 77 & 1 & 1 & 1 & 1 \\
\hline monkish2 & 8 & 1 & 77 & 1 & 38 & 1 & 38 \\
\hline monkish3 & 8 & 1 & 77 & 1 & 28 & 512 & 56 \\
\hline
\end{tabular}

Table 7.12: $M V G U D$ run with 6 variables in the bound set on 8 variable Machine Learning Benchmarks with $90 \%$ don't cares 


\subsubsection{A Summary of the results of testing MISDOM on Machine Learning Benchmarks}

The results obtained from the testing of MISDOM on Machine Learning Benchmarks have proved that Multi-Colorings or Clique-Coverings will nearly always produce better decompositions for Machine Learning Functions. Two example decomposed functions are shown here to show how the $G$ functions and the $\mathrm{H}$ functions differ depending on whether a Multi-Coloring is used or a Graph Coloring is used.

Table 7.13 shows the $\mathrm{G}$ function and Table 7.14 shows the $\mathrm{H}$ function which were obtained by running $M V G U D$ on the Machine Learning benchmark psu_ch $47 f 0$ with five variables in the bound set. Tables 7.15 and 7.16 show the resulting G and $\mathrm{H}$ Tables respectively, for $M V G U D$ run on the Machine Learning Function psu_rnd3 with five variables in the bound set. These Tables show the actual output file from $M V G U D$. In these Tables the first column represents the decomposed function for MVGUD run with MISDOM, and the second column represents the decomposed function for $M V G U D$ run with $D O M$. The output files are in the mublif format. In this format:

.type: Stands for the type of file.

.i: Stands for the number of inputs.

.o: Stands for the number of outputs.

.ilb: Stands for the names of the inputs.

.ob: Stands for the names of the outputs.

.imv: Represents the maximum multi-value of each input.

.omv: Represents the maximum multi-value of each output.

.p: Stands for the number of cubes in the block. From the G Tables 7.13 and 7.15 the generalized don't cares can be seen in the output for when $M V G U D$ is run with MISDOM. 


\begin{tabular}{|c|c|c|c|c|c|c|c|c|c|c|c|}
\hline \multicolumn{6}{|c|}{$M V G U D$ run with $M I S D O M$} & \multicolumn{6}{|c|}{$M V G U D$ run with $D O M$} \\
\hline \multicolumn{6}{|c|}{$\begin{array}{l}. \text { type } \mathrm{mv} \\
. \mathrm{i} 5 \\
.01 \\
. \mathrm{ilb} \mathrm{a} 0 \mathrm{a} 1 \mathrm{a} 2 \mathrm{a} 4 \mathrm{a} 5 \\
. \text { ob s2.0 } \\
. \mathrm{imv} 222222 \\
.0 \mathrm{mv} 4 \\
. \mathrm{p} 26\end{array}$} & \multicolumn{6}{|c|}{ 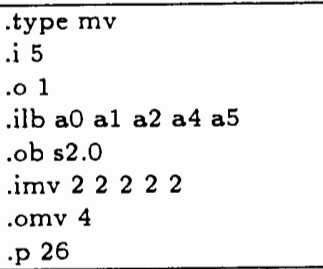 } \\
\hline 0 & 1 & 0 & 1 & 0 & 1 & 0 & & 0 & 1 & & 0 \\
\hline 0 & 1 & 0 & 1 & 1 & 3 & 0 & 1 & 0 & 1 & 1 & 3 \\
\hline 0 & 1 & 0 & 0 & 0 & 3 & 0 & 1 & 0 & 0 & 0 & 3 \\
\hline 0 & 1 & 1 & 1 & 0 & 2 & 0 & 1 & 1 & 1 & 0 & 1 \\
\hline 0 & 1 & 1 & 1 & 1 & 0,3 & 0 & 1 & 1 & 1 & 1 & 2 \\
\hline 0 & 1 & 1 & 0 & 0 & 3 & 0 & 1 & 1 & 0 & 0 & 3 \\
\hline 0 & 1 & 1 & 0 & 1 & 0,3 & 0 & 1 & 1 & 0 & 1 & 2 \\
\hline 0 & 0 & 0 & 1 & 0 & - & 0 & 0 & 0 & 1 & 0 & 0 \\
\hline 0 & 0 & 0 & 1 & 1 & 2 & 0 & 0 & 0 & 1 & 1 & 1 \\
\hline 0 & 0 & 0 & 0 & 0 & 3 & 0 & 0 & 0 & 0 & 0 & 3 \\
\hline 0 & 0 & 0 & 0 & 1 & 2,3 & 0 & 0 & 0 & 0 & 1 & 1 \\
\hline 0 & 0 & 1 & 1 & 0 & 0 & 0 & 0 & 1 & 1 & 0 & 2 \\
\hline 0 & 0 & 1 & 1 & 1 & 1,2 & 0 & 0 & 1 & 1 & 1 & 0 \\
\hline 0 & 0 & 1 & 0 & 0 & 3 & 0 & 0 & 1 & 0 & 0 & 3 \\
\hline 0 & 0 & 1 & 0 & 1 & 3 & 0 & 0 & 1 & 0 & 1 & 3 \\
\hline 1 & 1 & 0 & 1 & 0 & 3 & 1 & 1 & 0 & 1 & 0 & 3 \\
\hline 1 & 1 & 0 & 0 & 1 & 3 & 1 & 1 & 0 & 0 & 1 & 3 \\
\hline 1 & 1 & 1 & 1 & 0 & 3 & 1 & 1 & 1 & 1 & 0 & 3 \\
\hline 1 & 1 & 1 & 1 & 1 & 0,3 & 1 & 1 & 1 & 1 & 1 & 2 \\
\hline 1 & 0 & 0 & 1 & 1 & 3 & 1 & 0 & 0 & 1 & 1 & 3 \\
\hline 1 & 0 & 0 & 0 & 0 & 2,3 & 1 & 0 & 0 & 0 & 0 & 1 \\
\hline 1 & 0 & 0 & 0 & 1 & 1 & 1 & 0 & 0 & 0 & 1 & 0 \\
\hline 1 & 0 & 1 & 1 & 0 & 3 & 1 & 0 & 1 & 1 & 0 & 3 \\
\hline 1 & 0 & 1 & 1 & 1 & 3 & 1 & 0 & 1 & 1 & 1 & 3 \\
\hline 1 & 0 & 1 & 0 & 0 & 0 & 1 & 0 & 1 & 0 & 0 & 2 \\
\hline $\begin{array}{l}1 \\
\text {.er }\end{array}$ & & 1 & 0 & 1 & 1,2 & $\begin{array}{l}1 \\
\text {.er }\end{array}$ & & 1 & 0 & 1 & 0 \\
\hline
\end{tabular}

Table 7.13: Resulting G Function for $M V G U D$ run on Benchmark psu_ch $47 f 0$ 


\begin{tabular}{|c|c|c|c|c|c|c|c|c|c|}
\hline \multicolumn{5}{|c|}{ 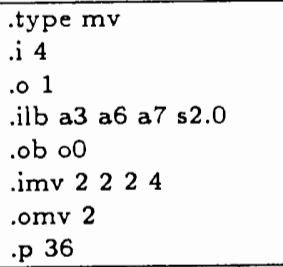 } & \multicolumn{5}{|c|}{$\begin{array}{l}\text {.type mv } \\
\text {.i } 4 \\
. \circ 1 \\
. \text { ilb a } 3 \text { a } 6 \text { a } 7 \text { s } 2.0 \\
.0 b \text { o0 } \\
\text {. imv } 22224 \\
. o m v 2 \\
. p 36\end{array}$} \\
\hline 1 & 0 & 0 & 0,3 & 0 & 0 & & 0 & 2 & 0 \\
\hline 0 & 0 & 0 & 2 & 1 & 0 & 0 & 0 & 1 & 1 \\
\hline 0 & 1 & 1 & 1 & 1 & 0 & 0 & 0 & 0 & 1 \\
\hline 1 & 0 & 1 & 0,3 & 0 & 0 & 0 & 1 & 2 & 0 \\
\hline 0 & 1 & 0 & 1,2 & 0 & 1 & 0 & 1 & 2 & 0 \\
\hline 0 & 1 & 0 & 0,3 & 0 & 1 & 1 & 0 & 1 & 0 \\
\hline 0 & 1 & 0 & 2 & 0 & 1 & 0 & 0 & 2 & 0 \\
\hline 0 & 0 & 1 & 0,3 & 0 & 0 & 1 & 0 & 0 & 0 \\
\hline 0 & 1 & 1 & 2,3 & 0 & 0 & 1 & 1 & 0 & 1 \\
\hline 1 & 0 & 1 & 3 & 0 & 1 & 1 & 1 & 3 & 0 \\
\hline 1 & 0 & 1 & 0 & 0 & 1 & 0 & 0 & 1 & 1 \\
\hline 1 & 1 & 0 & 2,3 & 0 & 1 & 0 & 1 & 3 & 0 \\
\hline 1 & 1 & 1 & - & 0 & 1 & 0 & 1 & 1 & 0 \\
\hline 0 & 1 & 1 & 0 & 1 & 1 & 0 & 0 & 3 & 0 \\
\hline 1 & 1 & 1 & 2,3 & 0 & 0 & 0 & 0 & 2 & 0 \\
\hline 0 & 0 & 0 & 1 & 1 & 0 & 1 & 0 & 1 & 0 \\
\hline 1 & 0 & 0 & 2 & 1 & 0 & 1 & 1 & 2 & 1 \\
\hline 1 & 1 & 1 & 3 & 0 & 0 & 1 & 1 & 1 & 0 \\
\hline 1 & 0 & 1 & 2,3 & 0 & 1 & 1 & 1 & 0 & 0 \\
\hline 1 & 0 & 0 & 3 & 0 & 0 & 1 & 1 & 3 & 0 \\
\hline 0 & 0 & 0 & 0 & 0 & 1 & 1 & 0 & 3 & 0 \\
\hline 0 & 1 & 1 & 2 & 0 & 1 & 0 & 1 & 0 & 1 \\
\hline 0 & 0 & 0 & 0,3 & 0 & 1 & 0 & 0 & 0 & 1 \\
\hline 0 & 0 & 1 & 1 & 1 & 0 & 0 & 1 & 0 & 1 \\
\hline 1 & 1 & 1 & 1,2 & 0 & 0 & 0 & 1 & 1 & 1 \\
\hline 0 & 1 & 1 & 3 & 0 & 0 & 1 & 0 & 3 & 0 \\
\hline 0 & 0 & 1 & 1,2 & 1 & 1 & 1 & 1 & 1 & 0 \\
\hline 1 & 0 & 1 & 1 & 1 & 0 & 0 & 1 & 3 & 0 \\
\hline 1 & 0 & 0 & 1,2 & 1 & 0 & 0 & 0 & 3 & 0 \\
\hline 1 & 1 & 0 & 3 & 0 & 1 & 1 & 0 & 2 & 1 \\
\hline 0 & 0 & 1 & 2 & 1 & & & & & \\
\hline 0 & 1 & 0 & 3 & 0 & & & & & \\
\hline 1 & 1 & 1 & 2 & 0 & & & & & \\
\hline 0 & 0 & 1 & 3 & 0 & & & & & \\
\hline 0 & 0 & 0 & 3 & 0 & & & & & \\
\hline & & 0 & 0 & 1 & & & & & \\
\hline
\end{tabular}

Table 7.14: Resulting H Function for MVGUD run on Benchmark psu_ch $47 f 0$ 


\begin{tabular}{|c|c|c|c|c|c|c|c|c|c|c|c|}
\hline \multicolumn{6}{|c|}{$M V G U D$ run with $M I S D O M$} & \multicolumn{6}{|c|}{$M V G U D$ run with $D O M$} \\
\hline \multicolumn{6}{|c|}{ 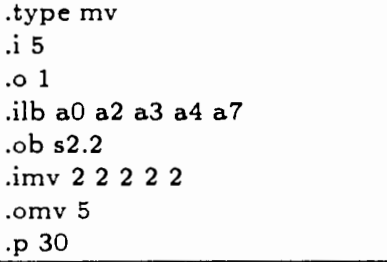 } & \multicolumn{6}{|c|}{$\begin{array}{l}\text {.type } \mathrm{mv} \\
. \text { i } 5 \\
.01 \\
. \text { ilb a0 a2 a3 a4 a7 } \\
. \text { ob s2.2 } \\
. \text { imv } 222222 \\
. \text { omv } 5 \\
. \mathrm{p} 31\end{array}$} \\
\hline 0 & 1 & 0 & 1 & 0 & 4 & 0 & 1 & 0 & 1 & & 1 \\
\hline 0 & 1 & 0 & 1 & 1 & 3 & 0 & 1 & 0 & 1 & 1 & 0 \\
\hline 0 & 1 & 0 & 0 & 0 & 4 & 0 & 1 & 0 & 0 & 0 & 1 \\
\hline 0 & 1 & 0 & 0 & 1 & 4 & 0 & 1 & 0 & 0 & 1 & 1 \\
\hline 0 & 1 & 1 & 1 & 0 & 2 & 0 & 1 & 1 & 1 & 0 & 4 \\
\hline 0 & 1 & 1 & 1 & 1 & 3 & 0 & 1 & 1 & 1 & 1 & 0 \\
\hline 0 & 1 & 1 & 0 & 0 & 3 & 0 & 1 & 1 & 0 & 0 & 0 \\
\hline 0 & 1 & 1 & 0 & 1 & $0,2,4$ & 0 & 1 & 1 & 0 & 1 & 3 \\
\hline 0 & 0 & 0 & 1 & 0 & 3 & 0 & 0 & 0 & 1 & 0 & 2 \\
\hline 0 & 0 & 0 & 1 & 1 & 3 & 0 & 0 & 0 & 1 & 1 & 2 \\
\hline 0 & 0 & 0 & 0 & 0 & $0,2,4$ & 0 & 0 & 0 & 0 & 0 & 0 \\
\hline 0 & 0 & 0 & 0 & 1 & 2 & 0 & 0 & 0 & 0 & 1 & 2 \\
\hline 0 & 0 & 1 & 1 & 0 & $0,1,2$ & 0 & 0 & 1 & 1 & 0 & 1 \\
\hline 0 & 0 & 1 & 1 & 1 & $0,1,2$ & 0 & 0 & 1 & 1 & 1 & 3 \\
\hline 0 & 0 & 1 & 0 & 0 & 0,1 & 0 & 0 & 1 & 0 & 0 & 0 \\
\hline 0 & 0 & 1 & 0 & 1 & 1,3 & 0 & 0 & 1 & 0 & 1 & 3 \\
\hline 1 & 1 & 0 & 1 & 0 & 0 & 1 & 1 & 0 & 1 & 0 & 1 \\
\hline 1 & 1 & 0 & 1 & 1 & 0 & 1 & 1 & 0 & 1 & 1 & 1 \\
\hline 1 & 1 & 0 & 0 & 0 & $0,2,4$ & 1 & 1 & 0 & 0 & 0 & 2 \\
\hline 1 & 1 & 0 & 0 & 1 & 3,4 & 1 & 1 & 0 & 0 & 1 & 2 \\
\hline 1 & 1 & 1 & 1 & 0 & 4 & 1 & 1 & 1 & 1 & 1 & 0 \\
\hline 1 & 1 & 1 & 1 & 1 & 1,2 & 1 & 1 & 1 & 0 & 0 & 4 \\
\hline 1 & 1 & 1 & 0 & 0 & 3 & 1 & 1 & 1 & 0 & 1 & 1 \\
\hline 1 & 1 & 1 & 0 & 1 & 0,4 & 1 & 0 & 0 & 1 & 0 & 1 \\
\hline 1 & 0 & 0 & 1 & 1 & 1 & 1 & 0 & 0 & 1 & 1 & 1 \\
\hline 1 & 0 & 0 & 0 & 0 & 1 & 1 & 0 & 0 & 0 & 0 & 1 \\
\hline 1 & 0 & 0 & 0 & 1 & 0 & 1 & 0 & 0 & 0 & 1 & 3 \\
\hline 1 & 0 & 1 & 1 & 0 & 1,3 & 1 & 0 & 1 & 1 & 0 & 3 \\
\hline 1 & 0 & 1 & 0 & 0 & 3,4 & 1 & 0 & 1 & 1 & 1 & 3 \\
\hline & 0 & 1 & 0 & 1 & 0,2 & 1 & 0 & 1 & 0 & 0 & 0 \\
\hline \multicolumn{6}{|c|}{.end } & & .end & 1 & 0 & 1 & 0 \\
\hline
\end{tabular}

Table 7.15: Resulting G Function for MVGUD run on Benchmark psu_rnd3 


\begin{tabular}{|c|c|c|c|c|c|c|c|c|c|}
\hline \multicolumn{5}{|c|}{$\begin{array}{l}\text { type mv } \\
. \text { i } 4 \\
.01 \\
. i l b \text { a } 1 \text { a } 5 \text { a } 6 \text { s } 2.2 \\
.0 b \text { o } \\
. i m v 2225 \\
. o m v 2 \\
. p 51\end{array}$} & \multicolumn{5}{|c|}{$\begin{array}{l}. \text { type } \mathrm{mv} \\
.14 \\
.01 \\
. \text {.ilb a4 a6 a } 7 \mathrm{~s} 2.0 \\
.0 \mathrm{~b} \text { o0 } \\
. \text { imv } 2225 \\
.0 \mathrm{mv} 2 \\
. \mathrm{p} 51\end{array}$} \\
\hline 0 & 0 & 1 & $0,1,2$ & 1 & 1 & & 1 & 3 & 1 \\
\hline 0 & 0 & 1 & 1,2 & 1 & 0 & 0 & 1 & 0 & 0 \\
\hline 1 & 1 & 1 & 0 & 1 & 1 & 0 & 0 & 2 & 1 \\
\hline 0 & 0 & 0 & 1,3 & 1 & 1 & 1 & 1 & 1 & 0 \\
\hline 1 & 0 & 1 & 4 & 1 & 1 & 0 & 1 & 0 & 0 \\
\hline 1 & 0 & 0 & 1,3 & 1 & 0 & 0 & 1 & 2 & 1 \\
\hline 1 & 1 & 0 & 4 & 1 & 0 & 1 & 1 & 0 & 0 \\
\hline 1 & 1 & 0 & 0 & 1 & 0 & 1 & 0 & 4 & 0 \\
\hline 1 & 0 & 1 & 1 & 1 & 1 & 1 & 1 & 2 & 1 \\
\hline 0 & 1 & 0 & 0 & 0 & 1 & 1 & 0 & 1 & 1 \\
\hline 1 & 0 & 0 & 0,1 & 1 & 1 & 1 & 0 & 4 & 0 \\
\hline 0 & 1 & 0 & $0,2,4$ & 0 & 0 & 0 & 0 & 1 & 1 \\
\hline 1 & 1 & 1 & 3 & 0 & 0 & 1 & 0 & 1 & 0 \\
\hline 0 & 0 & 1 & 2 & 1 & 1 & 0 & 0 & 1 & 1 \\
\hline 1 & 0 & 1 & 2 & 1 & 0 & 0 & 0 & 4 & 1 \\
\hline 1 & 1 & 1 & 4 & 0 & 0 & 1 & 1 & 2 & 1 \\
\hline 1 & 1 & 0 & 2 & 0 & 0 & 1 & 1 & 1 & 1 \\
\hline 1 & 1 & 0 & 3 & 1 & 1 & 0 & 0 & 4 & 0 \\
\hline 1 & 1 & 0 & 1,2 & 0 & 0 & 0 & 1 & 3 & 0 \\
\hline 0 & 1 & 1 & 2 & 1 & 0 & 0 & 0 & 2 & 0 \\
\hline 1 & 0 & 0 & 4 & 0 & 0 & 0 & 0 & 3 & 1 \\
\hline 0 & 1 & 0 & 1,3 & 1 & 1 & 0 & 1 & 3 & 1 \\
\hline 0 & 1 & 1 & 3 & 1 & 1 & 1 & 1 & 0 & 0 \\
\hline 0 & 1 & 0 & 4 & 0 & 0 & 1 & 0 & 2 & 1 \\
\hline 1 & 0 & 1 & 0 & 0 & 1 & 0 & 1 & 1 & 1 \\
\hline 0 & 0 & 0 & 3 & 1 & 1 & 0 & 0 & 0 & 0 \\
\hline 0 & 0 & 0 & 4 & 0 & 0 & 1 & 1 & 3 & 0 \\
\hline 1 & 0 & 0 & 1 & 1 & 1 & 1 & 0 & 3 & 0 \\
\hline 0 & 0 & 1 & 0,2 & 1 & 0 & 0 & 1 & 1 & 0 \\
\hline 0 & 1 & 1 & 1,3 & 1 & 1 & 0 & 0 & 3 & 0 \\
\hline 0 & 0 & 1 & 3,4 & 0 & 1 & 1 & 0 & 0 & 1 \\
\hline 1 & 0 & 0 & 2 & 0 & 1 & 0 & 1 & 2 & 1 \\
\hline 0 & 1 & 1 & 0 & 0 & 0 & 1 & 0 & 0 & 0 \\
\hline 1 & 1 & 0 & 1 & 0 & 1 & 1 & 1 & 4 & 0 \\
\hline 1 & 1 & 1 & 0,2 & 1 & 1 & 0 & 1 & 4 & 1 \\
\hline 0 & 1 & 0 & 1 & 1 & 0 & 0 & 0 & 0 & 0 \\
\hline 0 & 0 & 0 & 0 & 0 & 0 & 1 & 0 & 3 & 0 \\
\hline 0 & 0 & 1 & 0,1 & 1 & & & & & \\
\hline 1 & 0 & 0 & 3 & 1 & & & & & \\
\hline 0 & 1 & 1 & 0,4 & 0 & & & & & \\
\hline 0 & 1 & 1 & 4 & 0 & & & & & \\
\hline 1 & 1 & 1 & $0,1,2$ & 1 & & & & & \\
\hline 0 & 1 & 0 & 0,2 & 0 & & & & & \\
\hline 0 & 1 & 0 & 2 & 0 & & & & & \\
\hline 0 & 0 & 1 & 0 & 1 & & & & & \\
\hline 0 & 1 & 0 & 3 & 1 & & & & & \\
\hline 0 & 0 & 1 & 4 & 0 & & & & & \\
\hline 1 & 0 & 1 & 3 & 0 & & & & & \\
\hline 1 & 0 & 0 & 0 & 1 & & & & & \\
\hline 0 & 0 & 0 & $0,2,4$ & 0 & & & & & \\
\hline $\begin{array}{l}0 \\
\text { e }\end{array}$ & & 1 & 3 & 0 & & & & & \\
\hline
\end{tabular}

Table 7.16: Resulting H Function for MVGUD run on Benchmark psu_rnd3 


\subsection{Summary and some Conclusions based on the results obtained in Chapter 7}

The results obtained in this Chapter have provided some deeper insights into the decomposition process. The first very important conclusion is that MultiColorings or Clique-Coverings will nearly always produce better decompositions for Machine Learning Functions. Also the Multi-Coloring has proved that Decomposition of Relations is a very powerful concept, and why the introduction of generalized don't cares can improve the quality of the decompositions achieved. Whenever there are generalized don't cares in the output of a Relation, the simplest circuit can be selected, and implemented. While in the case of a function, once the decomposition is done there is no further choice that can be made. Hence while decomposing a function, generalized don't cares must be introduced, and that is what the Multi-Coloring is doing.

From Tables 7.5, 7.6 7.7 and 7.8 it was seen that as the number of variables in the Bound Set increases the number of generalized don't cares introduced in the decomposed blocks of the function increases. This can be seen from Figure 7.5 and Figure 7.6. In Figures 7.5 and 7.6 the number of variables in the Bound Set are increased along the $\mathrm{X}$-axis, and the $\mathrm{Y}$-axis displays the average NOFP or $C O S C$. In order to get the average NOFP, for a constant number of variables in the Bound Set, the NOFP was added and then divided by the total number of program runs. Average COSC was calculated in the same way. Figure 7.5 is for when MVGUD was run on the Machine Learning Benchmarks with $70 \%$ of don't-cares, and Figure 7.6 is for when MVGUD was run on the Machine Learning Benchmarks with $90 \%$ of don't-cares. From these graphs the following observations can be made:

Observation 7.1 As the number of variables in the Bound Set increases for a constant number of don't cares, NOFP increases exponentially.

Observation 7.2 As NOFP increases, COSC increases. 


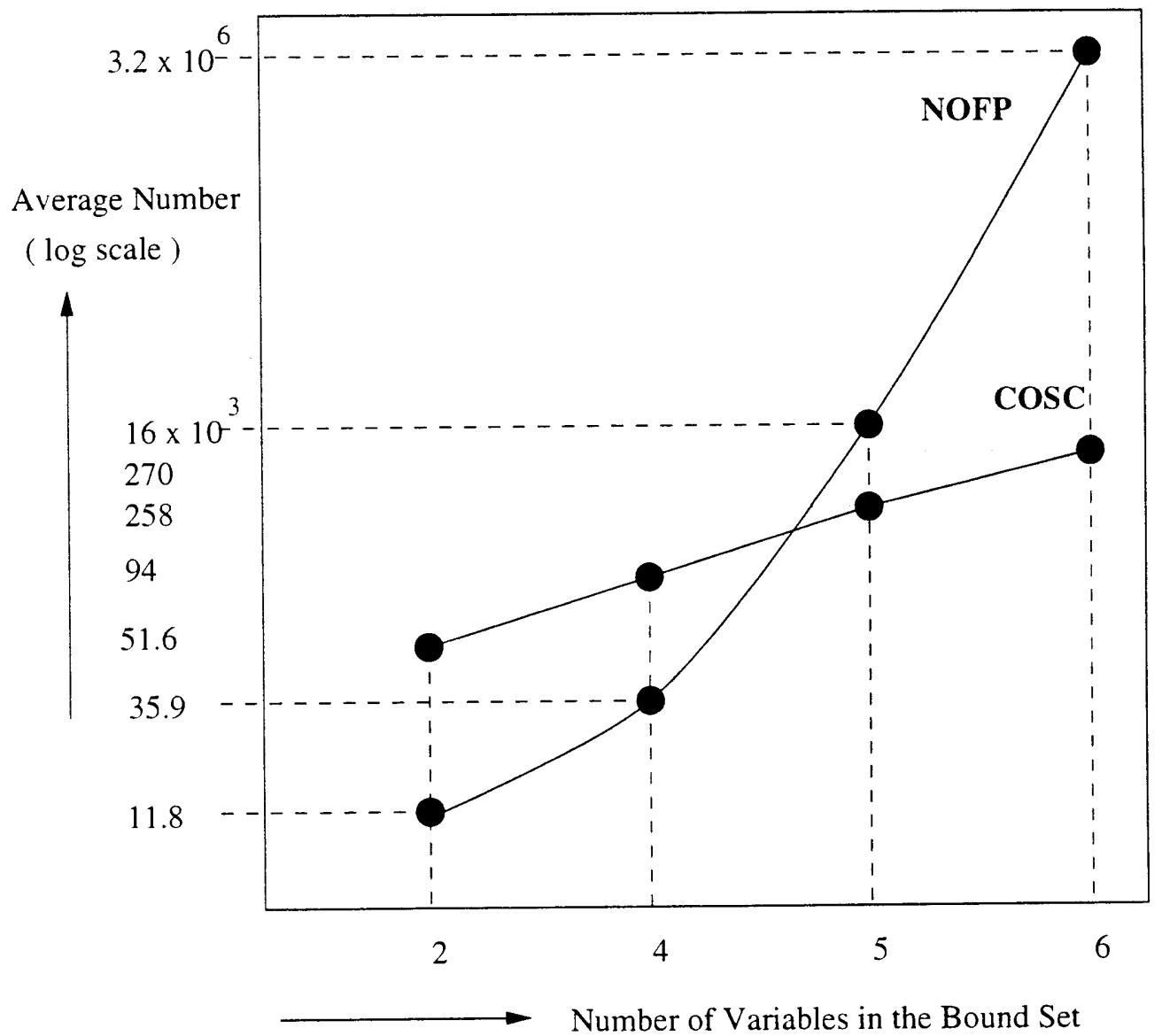

Figure 7.5: A Graph for 8 variable Machine Learning Benchmarks with $70 \%$ don't cares showing how $N O F P$ and $C O S C$ vary with increasing number of variables in the Bound set 


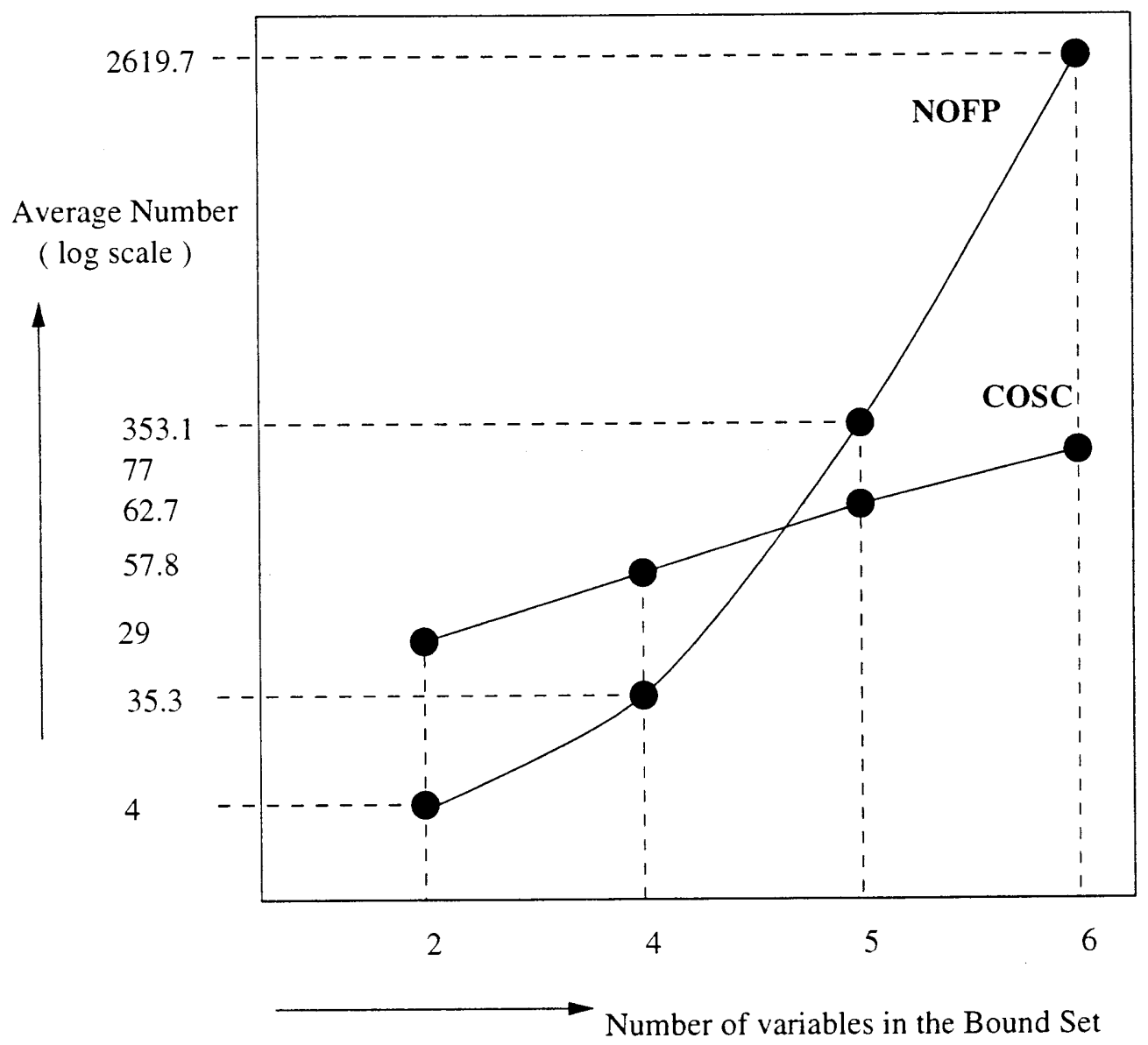

Figure 7.6: A Graph for 8 variable Machine Learning Benchmarks with $90 \%$ don't cares showing how $N O F P$ and $C O S C$ vary with increasing number of variables in the Bound set 
It was expected that as the number of don't-cares increase, NOFP should increase, but this was not so. The reason for this is that as the number of don't cares increase the size of the $\mathrm{G}$ and the $\mathrm{H}$ Tables is reducing, hence these reduced Tables may have a higher density of generalized don't cares than for the Tables with $70 \%$ don't cares, but due to the larger size of the Tables with $70 \%$ of don't cares they will have more generalized don't cares.

Observations 7.1 and 7.2 suggest that, in order to get better decompositions, it would be a good idea to have a new strategy for decomposition which starts from larger Bound Sets and then decomposes each $\mathrm{G}$ relation or function obtained. I think that this will lead to better decompositions. In order to try this new Strategy MVGUD will have to be modified. This would be one of the future works of the Functional Decomposition Group. The next Chapter concludes this thesis, and illustrates what I think the future work of the Functional Decomposition group should be. 
CHAPTER 8

\section{MY CONTRIBUTIONS TO THE FUNCTIONAL DECOMPOSITION GROUP, CONCLUSIONS AND FUTURE WORK}

Since the Functional Decomposition Group was formed at Portland State University, we have spent a lot of time researching the different aspects of Functional Decomposition. Everybody in the group was involved in a different aspect of Functional Decomposition. My part was investigating the Column Multiplicity problem of Functional Decomposition. In order to investigate the Column Multiplicity problem properly, it was necessary to do it very systematically in a number of steps. As the first step I programmed the heuristic Dominance Coloring program and compared it with the heuristic Clique Partitioning. This testing showed us that a Clique Partitioning and a Graph Coloring both achieve nearly the same decompositions, and none of them proves to be better then the other. Since this did not solve the Column Multiplicity problem for us as the next step I wrote an Exact Graph Coloring. This provided us with a deeper insight into the Column Multiplicity problem. This testing proved to us that Exact Algorithms are not needed to find the Column Multiplicity for Curtis Decompositions. This was a very important result because it was always thought that an Exact Algorithm can significantly improve the decomposition. Even though this result provided us with a very useful idea, it also raised the very essential question that why is the Exact Algorithm unable to provide better decompositions? In order to find out the answer to this question, I tested DOM,CLIP and EXOC on the same graphs, generated during the process of Functional Decomposition in Chapter 5. This experiment told us that the graphs generated during the decomposition process 
are much simpler than graphs generated randomly. This was another important conclusion, because it provided us with a deeper insight into the entire decomposition process, and not only to the part of finding the Column Multiplicity.

On showing that an Exact Graph Coloring is not necessary in Decomposition, the next question was whether a Multi-Coloring would improve the quality of decompositions with respect to coloring? In order to test the quality of the results obtained by using a Multi-Coloring, I developed two new cost functions. Then I developed and programmed a new algorithm for Multi-Coloring and tested it on MCNC and Machine Learning Benchmarks. Then I compared the heuristic Multi-Coloring with a heuristic Graph Coloring in decomposition of Circuit and Machine Learning Benchmarks. The two programs were evaluated by using the two new cost functions. The results of this experiment showed that a Multi-Coloring can improve the quality of decompositions achieved, for Machine Learning Functions. Thus I showed that in nearly all cases it is better to have a Multi-Coloring than having a Graph Coloring to find the Column Multiplicity in Decomposition of functions and relations. I do not know if this thesis has solved the Column Multiplicity problem in Functional Decomposition, but I think it has provided some very valuable insights into the decomposition problem, and it provides a direction that should be proceeded along in order to solve the decomposition problem better. The future direction that I propose that should be gone along to solve the column multiplicity problem better in the Decomposition process is to use a Multi-Coloring to find the Column Multiplicity and to start from larger bound sets, creating $\mathrm{G}$ relations, and then decomposing these $\mathrm{G}$ relations. This thesis has taught me that the decomposition process is a very powerful process, but it still has to attain its right status in the industry. But I think the decomposition process once solved better will become a very powerful tool in industry and research. 


\section{BIBLIOGRAPHY}

[1] R.L. Ashenhurst, "The Decomposition of Switching functions," Proc. Int'l Symp.Theory of Switching Functions, pp.74-116,1959.

[2] H.A. Curtis, "A New Approach to the Design of Switching Circuits," Princeton, N.J., Van Nostrand, 1962.

[3] T. Luba, "New Approach to Boolean Function Decomposition for ROMs and PLAs," Publishers of Institute of Telecommunication, Warsaw Technical University, No.123, 1985.

[4] T.Luba, M. Mochocki, J. Rybnik, "Decomposition of Information Systems Using Decision Tables," Bulletin of the Polish Academy of Sciences, Technical Sciences, Vol. 41, No.3. 1993.

[5] W. Wan, M.A. Perkowski, "A New Approach to the Decomposition of Incompletely Specified Multi-Output Functions Based on Graph-Coloring and Local Transformations and its Application to FPGA mapping," Proc. of EURO-DAC'92, Hamburg, pp. 230 - 235, September, 1992.

[6] W. Wan, Thesis, "A New Approach to the Decomposition of Incompletely Specified Multi-Output Functions Based on Graph-Coloring and Local Transformations and its Application to FPGA mapping," Portland State University. 1992.

[7] M.A. Perkowski, T. Ross, D. Gadd, J.A. Goldman, and N. Song, "Application of ESOP Minimization in Machine Learning and Knowledge Discovery," Proc. Reed-Muller'95, pp. 102-109, 1995.

[8] J.P. Roth, "Minimization over Boolean Trees," IBM Journal.4, 5, pp. 543555, 1960. 
[9] R.M. Karp, F.E. McFarlin, J.P. Roth, J.R. Wilts, "A Computer Program for the Synthesis of Combinational Switching Circuits," In Proc AIEE Annual Symposium on Switching Circuits Theory, pp. 182-194, 1961.

[10] J.P. Roth, and E.G. Wagner, "Algebraic Topological Methods for the Synthesis of Switching Systems. Part III: Minimization of Non Singular Boolean Trees," IBM J. Res. Develop. Vol 3, Oct 1962.

[11] R.M. Karp, "Functional Decomposition and Switching Circuit Design," J. Soc. Industr. Appl. Math., Vol 11, No 2, pp. 291-335, June 1963.

[12] T. Sasao, "Functional Decomposition of PLAs," Proc of the Intern. Workshop on Logic Synthesis, Research Triangle Park, North Carolina, May 12-15, pp. 1987.

[13] M.A. Perkowski, H. Uong, "Generalized Decomposition of Incompletely Specified Multioutput, Multi-Valued Boolean Functions," Unpublished Manuscript, Department of Electrical Engineering, PSU 1987.

[14] M. Ciesielski, S. Yang, "A Generalized PLA Decomposition with Programmable Encoders," In the Proc. of the Intern. Workshop on Logic Synthesis, pp.1-13, May 1989.

[15] N.Sherwani, "Algorithms for VLSI Physical Design Automation".

[16] M. Axtell, T. Ross, "Pattern Theory in Algorithm Design", National Aerospace and Electronics Conf (NAECON), pp. May 1993.

[17] Y.T. Lai, M. Pedram, S. Sastry, "BDD-based Decomposition of Logic Functions With Application to FPGA Synthesis," Proc of 30th DAC, pp. 642-647, 1993.

[18] M.A. Perkowski, "A New Representation of Strongly Unspecified Switching Functions and it Application to Multi-Level AND/OR/EXOR Synthesis." Proc. Reed Muller'95 Workshop, Chiba, Japan, August 1995, pp. 143-151. 
[19] S. Baase, "Computer Algorithms, Introduction to Design and Analysis", Second Edition, pp 351-352.

[20] J.C. Muzio, T.C. Wesselkamper, "Multiple-Valued Switching Theory," Adam Hilger, Boston, MA, 1986.

[21] P. Sapiecha, M. Perkowski, and T. Luba, "Decomposition of Information Systems Based on Graph Coloring Heuristics," Symposium on Modeling, Analysis and Simulation, CESA'96 IMACS Multiconference. Lille - France, July 9-12.

[22] S. Grygiel, M. Perkowski, M. Marek-Sadowska, T. Luba, L. Jozwiak, "Cube Diagram Bundles: A New Representation of Strongly Unspecified MultipleValued Functions and Relations," ISMVL'97.

[23] M. Perkowski, M. Marek-Sadowska, T. Luba, S. Grygiel, M. Nowicka, P. Burkey, R. Malvi, Z. Wang, J. Zhang, and C. Stanley, "Fundamental Operations on Multiple-Valued Cube Diagram Bundles," Unpublished paper, Portland State University, 1997.

[24] M. Perkowski, M. Marek-Sadowska, T. Luba, S. Grygiel, P. Burkey, R. Malvi, Z. Wang, J. Zhang, and C. Stanley, "GUD-MV:Multi Level Decomposition of Multi-Valued Functions and Relations: Part I: Cube Diagram Bundles," Unpublished paper, Portland State University, 1997.

[25] M. Perkowski, M. Marek-Sadowska, L. Jozwiak, M. Nowicka, R. Malvi, Z. Wang, J. Zhang, "Decomposition of Multiple- Valued Relations" ISMVL'g7.

[26] M. Perkowski, T. Luba, S. Grygiel, P. Burkey, M. Burns, N. Iliev, M. Kolsteran, R. Lisanke, R. Malvi, Z. Wang, H. Wu, F. Yang, S. Zhou, and J. Zhang, "Unified Approach to Functional Decomposition of Switching Functions," Technical Report, Portland State University, 1995. 